\title{
DERIVED GLUING CONSTRUCTION OF CHIRAL ALGEBRAS
}

\author{
SHINTAROU YANAGIDA
}

\begin{abstract}
We discuss the gluing construction of class $\mathcal{S}$ chiral algebras in derived setting. The gluing construction in non-derived setting was introduced by Arakawa to construct a family of vertex algebras of which the associated varieties give genus zero Moore-Tachikawa symplectic varieties. Motivated by the higher genus case, we introduce a dg vertex algebra version $\mathrm{MT}_{\mathrm{ch}}$ of the category of Moore-Tachikawa symplectic varieties, where a morphism is given by a dg vertex algebra equipped with action of the universal affine vertex algebra, and composition of morphisms is given by the BRST reduction. We also show that the procedure taking the associated scheme of gives a functor from $\mathrm{MT}_{\mathrm{ch}}$ to the category $\mathrm{MT}$ of derived Moore-Tachikawa varieties, which would imply compatibility of gluing constructions in both categories.
\end{abstract}

Contents

0. Introduction 1

0.1. Backgrounds 1

0.2. Derived gluing of vertex algebras - Organization of the text 4

0.3. Global notation

1. Poisson algebras in dg setting 8

1.1. Dg convention 8

1.2. Algebraic structures in dg setting 10

1.3. Shifted Poisson algebras 15

1.4. Shifted Poisson structures for derived stacks 18

2. Derived Hamiltonian reduction and classical BRST complex 20

2.1. Derived Hamiltonian reduction 20

2.2. Classical BRST complex 24

2.3. Moore-Tachikawa varieties in derived setting 29

2.4. The case of $G$-equivariant Poisson algebras 30

3. Jet and arc spaces in derived setting

3.1. Jet and arc spaces for ordinary schemes 31

3.2. Jet and arc spaces for derived schemes 33

4. Li filtration and vertex Poisson algebras in derived setting 37

4.1. Dg vertex algebras 37

4.2. Dg vertex Li algebras 42

4.3. Dg vertex Poisson algebras $\quad 45$

4.4. Li filtration $\quad 50$

5. Coisson BRST reduction and gluing procedure for arc spaces 52

5.1. Coisson BRST complex

5.2. Coisson gluing procedure 54

6. Derived gluing of dg vertex algebras 56

6.1. Chiral BRST complex $\quad 56$

6.2. Chiral gluing procedure 59

6.3. The category $\mathrm{MT}_{\mathrm{ch}} \quad 59$

References

\section{INTRODUCTION}

0.1. Backgrounds. Let $G$ be a simply connected semisimple linear algebraic group over the complex field C. In $[A]$, Arakawa introduced a family of vertex algebras

$$
\mathbf{V}_{G, b}^{\mathcal{S}} \quad\left(b \in \mathbb{Z}_{\geq 1}\right)
$$

called the genus zero chiral algebras of class $\mathcal{S}$. They are designed as "chiral quantization" of genus zero Moore-Tachikawa symplectic varieties, and the design results in the associativity property of the family. The

Date: April 21, 2020.

This work is supported by JSPS KAKENHI Grant Number 19K03399. 
associativity is encoded by BRST reduction of vertex algebras, and this article concerns a technical issue on the reduction when one wants to study higher genus cases.

0.1.1. Moore-Tachikawa symplectic varieites. In order to explain the detail, let us give a short recollection on Moore-Tachikawa symplectic varieties. See also [A, §1] for a brief explanation.

In [MT12], concerning expected properties of Higgs branches of Sicilian theories, Moore and Tachikawa proposed a two-dimensional topological field theory which target holomorphic symplectic varieties. Mathematically speaking, they conjectured for each semisimple algebraic group $G$ over $\mathbb{C}$ the existence of a symmetric monoidal functor

$$
\eta_{G}: \mathrm{Bo}_{2} \longrightarrow \mathrm{HS}
$$

from the category $\mathrm{Bo}_{2}$ of 2-bordisms to the category $\mathrm{HS}$ of holomorphic symplectic varieties which satisfies some axioms.

The source category $\mathrm{Bo}_{2}$ is a familiar one in the context of topological field theory. We use the following description of $\mathrm{Bo}_{2}$ :

- An object is a closed oriented one-dimensional manifold, or a disjoint union of $S^{1}$ 's.

- A morphism from $B_{1}$ to $B_{2}$ is the diffeomorphism class of an oriented two-dimensional manifold $\Sigma$ with boundary $\left(-B_{1}\right) \sqcup B_{2}$. We denote by $\Sigma_{g, b}$ the class of an oriented surface of genus $g$ with $b$ boundary components. In particular, the tube $\Sigma_{0,2}$ represents the identity morphism id $S_{S^{1}}$.

- Composition of morphisms is given by gluing. In particular, we have

$$
\Sigma_{0, b^{\prime}} \circ \Sigma_{0, b}=\Sigma_{0, b+b^{\prime}-2} .
$$

Disjoint union $\sqcup$ gives $\mathrm{Bo}_{2}$ a symmetric monoidal structure.

The target category HS is described as follows.

- An object is a semisimple algebraic group over $\mathbb{C}$.

- A morphism from $G_{1}$ to $G_{2}$ is a possibly singular symplectic variety $X$ over $\mathbb{C}$ with a $\mathbb{C}^{\times}$-action scaling the symplectic form by weight 2 together with Hamiltonian action of $G_{1} \times G_{2}$ satisfying some regularity condition. The identity $\operatorname{id}_{G} \in \operatorname{Hom}_{\mathrm{HS}}(G, G)$ is the cotangent bundle $T^{*} G$ with the left and right multiplication of $G$.

- Composition of $X \in \operatorname{Hom}_{\mathrm{HS}}\left(G_{1}, G_{2}\right)$ and $X^{\prime} \in \operatorname{Hom}_{\mathrm{HS}}\left(G_{2}, G_{3}\right)$ is given by the Hamiltonian reduction of the product with respect to the diagonal $G_{2}$-action:

$$
X^{\prime} \circ X:=\left(X^{\mathrm{op}} \times X^{\prime}\right) / / \Delta\left(G_{2}\right)=\mu^{-1}(0) / \Delta\left(G_{2}\right) .
$$

Here $X^{\text {op }}$ denotes the symplectic variety $X$ with the opposite symplectic structure, and the morphism $\mu: X^{\mathrm{op}} \times X^{\prime} \rightarrow \mathfrak{g}_{2}^{*}$ is the momentum map $\mu(x, y):=-\mu_{X}(x)+\mu_{X^{\prime}}(y)$ with $\mu_{X}$ and $\mu_{X^{\prime}}$ the $\mathfrak{g}_{2}^{*}$ component of the momentum map $X \rightarrow \mathfrak{g}_{1}^{*} \times \mathfrak{g}_{2}^{*}$ and $X^{\prime} \rightarrow \mathfrak{g}_{2}^{*} \times \mathfrak{g}_{3}^{*}$ respectively. The reduction doesn't touch the $G_{1^{-}}$and $G_{3}$-actions, so that we have $X^{\prime} \circ X \in \operatorname{Hom}_{\mathrm{HS}}\left(G_{1}, G_{3}\right)$.

The cartesian product of groups and varieties gives HS a symmetric monoidal structure.

Let us denote

$$
W_{G}^{b}:=\eta_{G}\left(\Sigma_{0, b}\right),
$$

where $\Sigma_{0, b}$ is an oriented surface of genus 0 with $b$ boundary components. It encodes the genus zero part of the functor $\eta_{G}$. The functor $\eta_{G}$ should satisfy $\eta_{G}\left(S^{1}\right)=G$ and $W_{G}^{2}=T^{*} G$. We refer [MT12, §3] for the full axiom of $\eta_{G}$.

In [BFN], concerning mathematical construction of the Coulomb branches of three-dimensional supersymmetric gauge theory via perverse sheaves on affine Grassmannians, Braverman, Finkelberg and Nakajima constructed the genus zero Moore-Tachikawa varieties $W_{G}^{b}:=\eta_{G}\left(\Sigma_{0, b}\right)$ in a uniform way, and showed

$$
W_{G}^{1} \simeq G \times \mathcal{S}, \quad W_{G}^{2} \simeq T^{*} G, \quad W_{G}^{b^{\prime}} \circ W_{G}^{b} \simeq W_{G}^{b+b^{\prime}-2},
$$

where $\mathcal{S} \subset \mathfrak{g}^{*}$ denotes the Slodowy slice. The third isomorphism reflects the gluing $(0.2)$ in $\mathrm{Bo}_{2}$, and we call it the gluing condition. In particular, the genus zero part of the functor $\eta_{G}$ is established.

0.1.2. The $4 \mathrm{~d} / 2 \mathrm{~d}$ duality and chiral algebras of class $\mathcal{S}$. Next we explain the $4 \mathrm{~d} / 2 \mathrm{~d}$ duality.In $\left[\mathrm{BLL}^{+}\right], \mathrm{Beem}$ Lemos, Liendo, Peelaers, Rastelli and van Rees proposed a "functorial" construction

$$
\mathcal{T} \longmapsto V_{\mathcal{T}}
$$

of conformal vertex algebras $V_{\mathcal{T}}$ from a four-dimensional $\mathcal{N}=2$ superconformal field theory (4d SCFT for short) $\mathcal{T}$. Among such four-dimensional theories, we have the theory $\mathcal{T}_{G, \Sigma}^{\mathcal{S}}$ of class $\mathcal{S}$ attached to a complex semisimple algebraic group $G$ and a punctured Riemann surface $\Sigma$. We will not go into the detail and refer 
the exposition [Ta18] for mathematicians. The vertex algebra obtained from $\mathcal{T}_{G, \Sigma}^{\mathcal{S}}$ by the above "functor" is called the chiral algebras of class $\mathcal{S}$. We denote it by $V_{G, \Sigma}^{\mathcal{S}}$.

A clue to identify the vertex algebra $V_{G, \Sigma}^{\mathcal{S}}$ is to consider an "invariant" of the physical theory $\mathcal{T}_{G, \Sigma}^{\mathcal{S}}$. The attachment $\mathcal{T} \mapsto V_{\mathcal{T}}$ is one of such invariants. Another interesting invariant is the hyperkähler manifold $\mathcal{M}_{\text {Higgs }}(\mathcal{T})$ called the Higgs branch of $\mathcal{T}$. See [Ta18] for more information. In [BR18], Beem and Rastelli conjectured that for any $4 \mathrm{~d}$ SCFT $\mathcal{T}$ there is an isomorphism

$$
\mathcal{M}_{\text {Higgs }}(\mathcal{T}) \stackrel{?}{\simeq} \operatorname{Specm}\left(R_{V_{\mathcal{T}}}\right)
$$

of holomorphic symplectic varieties, where the right hand side denotes the associated variety of the vertex algebra $V_{\mathcal{T}}$. See $\S 0.1 .3$ for more information, and Definition 4.4.5 for the precise definition of $R_{V_{\mathcal{T}}}$.

Now we can explain the result in $[\mathrm{A}]$. Arakawa considered the following "chiral quantization" $\eta_{G}^{\mathrm{VA}}$ of the Moore-Tachikawa functor $\eta_{G}$. Let us denote by $V_{k}(\mathfrak{g})$ the universal affine vertex algebra at level $k$ for $\mathfrak{g}=\operatorname{Lie}(G)$, and by $h^{\vee}$ the dual Coxeter number of $\mathfrak{g}$ (see $\S 4.1 .4$ for the detail). He considered a symmetric monoidal functor

$$
\eta_{G}^{\mathrm{VA}}: \mathrm{Bo}_{2} \longrightarrow \mathrm{VA},
$$

where the category VA is roughly explained as follows. See $\S 6.2$ for the detail.

- An object is a semisimple algebraic group.

- A morphism from $G_{1}$ to $G_{2}$ is a vertex algebra $V$ equipped with a vertex algebra morphism $V_{-h_{1}^{\vee}}\left(\mathfrak{g}_{1}\right) \otimes$ $V_{-h_{2}^{\vee}}\left(\mathfrak{g}_{2}\right) \rightarrow V$ and satisfying some conditions.

- Composition of $V \in \operatorname{Hom}_{\mathrm{VA}}\left(G_{1}, G_{2}\right)$ and $V^{\prime} \in \operatorname{Hom}_{\mathrm{VA}}\left(G_{2}, G_{3}\right)$ is given by the relative BRST reduction:

$$
V^{\prime} \circ V:=H^{\frac{\infty}{2}+0}\left(\widehat{\mathfrak{g}}_{-2 h_{2}^{\vee}}, \mathfrak{g}_{2}, V^{\mathrm{op}} \otimes V^{\prime}\right) .
$$

The condition on $\eta_{G}^{\mathrm{VA}}$ is that for any surface $\Sigma \in \operatorname{Mor}\left(\mathrm{Bo}_{2}\right)$, the vertex algebra $\eta_{G}^{\mathrm{VA}}(\Sigma)$ should coincide with $V_{\Sigma}^{\mathcal{S}}$, the chiral algebra of class $\mathcal{S}$.

Arakawa constructed in $[\mathrm{A}]$ the genus zero part of the functor $\eta_{G}^{\mathrm{VA}}$. In other words, he constructed the image

$$
\mathbf{V}_{G, b}^{\mathcal{S}}:=\eta_{G}^{\mathrm{VA}}\left(\Sigma_{0, b}\right)
$$

of the genus 0 surface $\Sigma_{0, b}$ with $b$ boundaries, and checked a "chiral quantization" of (0.3):

$$
\mathbf{V}_{G, 1}^{\mathcal{S}} \simeq H_{\mathrm{DS}}^{0}\left(\mathcal{D}_{G}^{\mathrm{ch}}\right), \quad \mathbf{V}_{G, 2}^{\mathcal{S}} \simeq \mathcal{D}_{G}^{\mathrm{ch}}, \quad \mathbf{V}_{G, b^{\prime}}^{\mathcal{S}} \circ \mathbf{V}_{G, b}^{\mathcal{S}} \simeq \mathbf{V}_{G, b+b^{\prime}-2}^{\mathcal{S}},
$$

Here $\mathcal{D}_{G}^{\text {ch }}$ denotes the algebra of chiral differential operators on $G$ at the critical level, and $H_{\mathrm{DS}}^{0}$ denotes the quantum Drinfeld-Sokolov reduction. The third relation is called the associativity in [A]. Moreover Arakawa showed

$$
R_{\mathbf{V}_{G, b}^{\mathcal{S}}}=\mathbb{C}\left[W_{G}^{b}\right]
$$

where the right hand side denotes the coordinate ring. Thus he solved the conjecture (0.5) for the genus zero class $\mathcal{S}$ theories $\mathcal{T}=\mathcal{T}_{\Sigma_{0, b}}^{\mathcal{S}}$.

0.1.3. Chiral quantization. So far we have used the word "chiral quantization" several times. Let us clarify what it means. Since it is also a good place to recall the associated schemes of vertex algebras, let us explain the chiralization of a Poisson structure.

(1) We start with a Poisson algebra $R$, i.e., a commutative algebra with a Poisson bracket. It corresponds to an affine Poisson scheme $\operatorname{Spec}(R)$.

(2) Recall the arc space, or the $\infty$-jet space, $J_{\infty}(Y)$ of a scheme $Y$. It is a scheme having the universal property

$$
\operatorname{Hom}_{\mathrm{Sch}}\left(\operatorname{Spec}(A), J_{\infty}(Y)\right)=\operatorname{Hom}_{\mathrm{Sch}}(\operatorname{Spec}(A[[t]]), Y)
$$

for any commutative algebra $A$, where Sch denotes the category of schemes. See $\S 3.1$ for the precise statement.

(3) By $[\mathrm{A} 15, \S 2.3]$, the coordinate $\operatorname{ring} J_{\infty}(R)$ of the arc space $J_{\infty}(\operatorname{Spec}(R))$ has a structure of vertex Poisson algebra induced naturally from the original Poisson algebra structure on $R$. See $\S 4.3$ for the detail.

(4) By [Li05], for a vertex algebra $V$, the associated graded space $\operatorname{gr}^{F} V$ with respect to the Li filtration $F^{\bullet} V$ has a structure of vertex Poisson algebra. The component

$$
R_{V}:=F^{0} V / F^{1} V
$$


is a Poisson algebra, which is called Zhu's $C_{2}$-algebra [Z96]. The corresponding affine Poisson scheme $X_{V}:=\operatorname{Spec}\left(R_{V}\right)$ is called the associated scheme of $V$. We have a natural surjective morphism $J_{\infty}\left(R_{V}\right) \rightarrow \operatorname{gr} V$ of vertex Poisson algebras. See $\S 4.4 .1$ for the detail.

Now we cite from [A, Definition 2.1] the terminology: Let $V$ be a vertex algebra. If Zhu's $C_{2}$-algebra $R_{V}$ is isomorphic to a Poisson algebra $R$, then we call $V$ a chiral quantization of the affine Poisson scheme $X=$ $\operatorname{Spec}(R)$. If moreover $V$ is separated (Definition 4.4.8), $R_{V}$ is reduced and the surjection $J_{\infty}\left(R_{V}\right) \rightarrow \operatorname{gr}^{F} V$ is an isomorphism, then $V$ is called a strict chiral quantization of $X$.

Using the terminology above, we can restate the relation $(0.8)$ as: The genus zero chiral algebra $\mathbf{V}_{G, b}^{\mathcal{S}}$ of class $\mathcal{S}$ is a strict chiral quantization of the genus zero Moore-Tachikawa varieties $W_{G}^{b}$. We also have a functorial picture

$$
\mathbf{V}_{G, b^{\prime}}^{\mathcal{S}} \circ \mathbf{V}_{G, b}^{\mathcal{S}} \underset{(0.7)}{\simeq} \mathbf{V}_{G, b+b^{\prime}-2}^{\mathcal{S}} \stackrel{R_{(-)}}{\longrightarrow} W_{G}^{b^{\prime}} \circ W_{G}^{b} \underset{(0.3)}{\simeq} W_{G}^{b+b^{\prime}-2},
$$

and in this sense we can say that the relations (0.7) are chiral quantization of $(0.3)$. We call this picture the compatibility of gluing constructions.

It is then natural to expect that the procedure $R_{(-)}$taking Zhu's $C_{2}$-algebra makes the following diagram commutative:

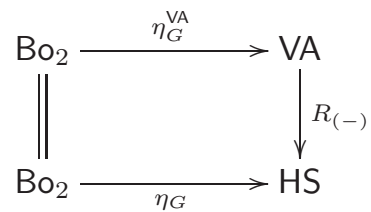

We can say that $[\mathrm{A}]$ established this commutativity restricting to the genus zero part of $\mathrm{Bo}_{2}$. His work can be regarded as a part of the mathematical formulation of the $4 \mathrm{~d} / 2 \mathrm{~d}$ duality "functor" $(0.4)$.

0.2. Derived gluing of vertex algebras - Organization of the text. The proof in [A] of the compatibility of gluing constructions for genus zero chiral algebras of class $\mathcal{S}$ is based on cohomology vanishing in the BRST reduction (0.6). As mentioned in the footnote in [A, p.3], there is a subtlety on this point in higher genus case. The issue is that the momentum map associated to the Hamiltonian action can be non-flat in higher genus case, for which we don't have a clean cohomology vanishing. As a result, we don't know explicit description of the chiral algebra $\mathbf{V}_{\Sigma}^{\mathcal{S}}$ of class $\mathcal{S}$ for a higher genus surface $\Sigma$ at this moment.

It was lucky for the author to take a lecture series by Arakawa on the article [A] in the end of November, 2019. In the lecture Arakawa suggested to use derived symplectic geometry to overcome this difficulty. The aim of this article is to give a first step to fulfill his suggestion.

The main materials in this text are as follows:

- We introduce a differential graded vertex algebra analogue

$$
\mathrm{MT}_{\mathrm{ch}}
$$

of the category MT of derived Moore-Tachikawa varieties. A morphism in $\mathrm{MT}_{\mathrm{ch}}$ is a differential graded vertex algebra $V$, and we denote by $V^{\prime} \widetilde{o} V$ a composition of morphisms.

- The compatibility of derived gluing constructions

$$
R_{\left(V^{\prime} \widetilde{\circ}\right)} \simeq R_{V^{\prime}} \widetilde{\circ} R_{V}
$$

for differential graded vertex algebras $V$ and $V^{\prime}$ of certain type. Here $R_{V}$ denotes Zhu's $C_{2}$-algebra of $V$ as for the ordinary vertex algebra, and the symbol $\simeq$ denotes quasi-isomorphism as homotopy Poisson algebras. Equivalently, the functor $R_{(-)}$induces

$$
R_{(-)}: \mathrm{MT}_{\mathrm{ch}} \longrightarrow \mathrm{MT} \text {. }
$$

Below we give an overview of this text along the line of the arguments in $§ 0.1 .3$. Let us start with the derived version of Poisson algebras. Hereafter we use the word "dg" to mean "differential graded".

0.2.1. Derived symplectic and Poisson geometry. The idea of using derived symplectic geometry to realize Moore-Tachikawa varieties is, as far as the author understands, originally due to Calaque [C15, Concluding remarks], [C14, Example 3.5]. He introduced a derived version of the category MT where composition of morphisms is given by the derived intersection of Lagrangian structures. This approach enables us to consider Hamiltonian reduction for non-flat momentum maps. See $\S 2.3$ for more information.

In this text we use affine derived Poisson geometry instead of derived symplectic geometry. Derived Poisson geometry was introduced in $[\mathrm{CPT}+]$ as a natural Poisson analogue of derived symplectic geometry [PTVV]. As in the ordinary Poisson and symplectic structures, we have an equivalence of non-degenerate 
Poisson and symplectic structures. See [C15, C14] for more information on derived symplectic geometry in the present context, and [S] for a review of derived Poisson geometry. The reason for us to use derived Poisson geometry is that Poisson structures appear naturally if we regard vertex algebras as chiral quantization, as we saw in $\S 0.1 .3$.

The aim of the beginning $\S 1$ is to give a recollection on shifted Poisson structures. Since what we need in this text is an affine version, we mainly treat those structures on commutative dg algebras. The main object is $\mathbb{P}_{n}$-algebra (Definition 1.3.1). The case $n=1$ corresponds to dg (non-shifted) Poisson algebra. Essentially we only need this $n=1$ case, but in order to introduce coisotropic structures to define derived Hamiltonian reduction later, we treat general shifted Poisson structures.

In the course of preparations in $\S \S 1.1-\S 1.2$, we explain notations on $\mathrm{dg}$ objects and algebraic structures on them. In particular, we denote by

$$
\mathrm{CE}(\mathfrak{l}, M)
$$

the Chevalley-Eilenberg complex for a dg Lie algebra $\mathfrak{l}$ and a $\operatorname{dg} \mathfrak{l}$-module $M$. See $§ 1.2 .4$, Definition 1.2.13 for the detail. Recall also that the correspondence $\mathrm{CE}(\mathfrak{l},-)$ is functorial, which will be used repeatedly in the following explanation.

We will also use the Kirillov-Kostant Poisson algebra: For a dg Lie algebra $\mathfrak{l}$, the symmetric algebra Sym(l) has a structure of $\mathrm{dg}$ Poisson algebra whose Poisson bracket is uniquely determined by the Lie bracket of $\mathfrak{l}$ and the Leibniz rule. See $\S 1.3 .3$ for the detail.

0.2.2. Derived Hamiltonian reduction of shifted Poisson algebra. We next consider a derived analogue of Hamiltonian reduction of shifted Poisson algebras. For the reduction of ordinary Poisson algebras, we refer [LPV, Chapter 5].

As mentioned in the previous $§ 0.2 .1$, Calaque [C14] formulated the gluing of Moore-Tachikawa varieties as derived intersection of Lagrangians in derived symplectic schemes with Hamiltonian group action. The corresponding procedure in the shifted Poisson structure is given by derived intersection of coisotropic structures introduced in $[\mathrm{CPT}+]$. In this text we use an equivalent but alternative approach of Safronov [S17], which will be reviewed in $\S 2$. This approach has an advantage in the point that the connection to the classical BRST complex is clear.

The coisotropic structure and Hamiltonian reduction is explained in $\S 2.1$. Let $\mathfrak{l}$ be a dg Lie algebra and $R$ a dg Poisson algebra. Noticing that $R$ can be regarded as a dg Lie algebra, we call a morphism $\mu: R \rightarrow \mathfrak{l}$ of $\mathrm{dg}$ Lie algebras a momentum map (see Definition 1.3.11 and Remark 1.3.12). For such $\mu$, the morphism $\mathrm{CE}(\mathfrak{l}, \mu)$ is coisotropic in the sense of Definition 2.1.3. Using coisotropic morphisms, we define

$$
R / /_{\mu}^{\mathbb{L}} \operatorname{Sym}(\mathfrak{l}):=\mathrm{CE}(\mathfrak{l}, \mathbb{k}) \bigotimes_{\mathrm{CE}(\mathfrak{l}, \operatorname{Sym} \mathfrak{l})}^{\mathbb{L}} \mathrm{CE}(\mathfrak{l}, R)
$$

and call it the derived Hamiltonian reduction of $R$ with respect to the momentum map $\mu$. It has a structure of $\widehat{\mathbb{P}}_{1}$-algebra, which can be restated as homotopy Poisson algebra (Definition 2.1.5).

The classical BRST complex, originally defined in non-dg setting by Kostant and Sternberg [KS87], will be introduced in $\S 2.2$. For the triple $(\mathfrak{l}, R, \mu)$ as above, we define the classical BRST complex $\operatorname{BRST}_{\mathrm{cl}}(\mathfrak{l}, R, \mu)$ by

$$
\operatorname{BRST}_{\mathrm{cl}}(\mathfrak{l}, R, \mu):=\left(\mathrm{CE}(\mathfrak{l}, \operatorname{Kos}(\mathfrak{l}, R, \mu)), d_{\mathrm{cl}}\right),
$$

where $\operatorname{Kos}(\mathfrak{l}, R, \mu)$ denotes the Koszul complex of $\mu^{-1}(0)$, and the differential $d_{\mathrm{BRST}}$ is given by the BRST charge. See Definition 2.2.3 for the detail.

The connection between the derived Hamiltonian reduction is explained in Proposition 2.2.8: For a finitedimensional Lie algebra $\mathfrak{l}$, we have an quasi-isomorphism

$$
R / / \mathbb{L}_{\mu}^{\mathbb{L}} \operatorname{Sym}(\mathfrak{l}) \underset{\text { qis }}{\simeq} \operatorname{BRST}_{\mathrm{cl}}(\mathfrak{g}, R, \mu)
$$

of homotopy Poisson algebras.

In $\S 2.3$, we introduce the category MT of derived Moore-Tachikawa varieties, which is essentially the same with the category introduced by Calaque [C14] mentioned before. An object is a semisimple algebraic group $G$, which is identified with its Lie algebra $\mathfrak{g}:=\operatorname{Lie}(G)$. A morphism from $G_{1}$ to $G_{2}$ is a non-degenerate $\widehat{\mathbb{P}}_{1}$-algebra $R$ with a momentum map $\mu_{R}=\mu_{R}^{1}+\mu_{R}^{2}: \mathfrak{g}_{1} \oplus \mathfrak{g}_{2} \rightarrow R$. Composition of $R \in \operatorname{Hom}_{\mathrm{MT}}\left(G_{1}, G_{2}\right)$ and $R^{\prime} \in \operatorname{Hom}_{\mathrm{MT}}\left(G_{2}, G_{3}\right)$ is given by

$$
R^{\prime} \widetilde{\circ}:=\operatorname{BRST}_{\mathrm{cl}}\left(\mathfrak{g}_{2}, R^{\mathrm{op}} \otimes R^{\prime}, \mu\right) \simeq\left(R^{\mathrm{op}} \otimes R^{\prime}\right) / /{ }_{\mu}^{\mathbb{L}} \operatorname{Sym}\left(\mathfrak{g}_{2}\right)
$$

with $\mu:=-\mu_{R}^{2}+\mu_{R^{\prime}}^{1}$. This is the derived gluing of Moore-Tachikawa varieties. See Definition 2.3.1 and Remark 2.3.2 for the detail. 
0.2.3. Jet and arc spaces for derived schemes, and $d g$ vertex Poisson algebras. As explained in $\S 0.1 .3$, in order to go intro the vertex world, we consider arc space of Poisson schemes. Thus, for the purpose of this text, we need a derived analogue of the theory of jet and arc spaces, which is a kind of exercise of derived algebraic geometry. Since there seems no explicit literature, we will give an explicit account it in $\S 3$.

For the explanation below, let us fix some notations. Let $R$ be a commutative dg algebra. Then there is a commutative dg algebra $J_{\infty}(R)$ such that $\operatorname{Hom}(R, A[[t]]) \simeq \operatorname{Hom}\left(J_{\infty}(R), A\right)$ for any commutative dg algebra $A$ (see Lemma 3.2.4 for the precise statement). We call it the arc space of $R$ (precisely speaking, we should call it the derived coordinate ring of the arc space). We have a natural embedding $R \hookrightarrow J_{\infty}(R)$.

In the context of derived gluing, we should consider a $\mathrm{dg}$ Poisson algebra $R$ and its arc space $J_{\infty}(R)$. The arc space inherits the Poisson structure of $R$, which should be of infinite-dimensional nature. As explained in $\S 0.1 .3$, for a non-dg Poisson algebra $R, J_{\infty}(R)$ has a structure of vertex Poisson algebra by the work of Arakawa $[A 12, \S 2.3]$. Thus we should study a dg version of vertex Poisson algebra. Such a notion is in fact included in the theory of coisson algebra introduced by Beilinson and Drinfeld [BD, 2.6]. In this text we explain a special case of their theory in $\S 4$.

We will explain standard notions on vertex algebras, vertex Poisson algebras and Li's canonical filtration in $\S 4$. Along the way, we also introduce dg versions of these "vertex" notions. Since these dg versions may not be standard, we list up the references of definitions:

- Dg vertex algebras: Definition 4.1.20.

- Dg modules over a dg vertex algebra: Definition 4.1.21.

- Dg vertex Poisson algebras: Definition 4.3.2.

- Li filtration of a dg vertex algebra: Definition 4.4.1.

In this introduction, the following statement is sufficient: For a dg Poisson algebra $R$, the arc space $J_{\infty}(R)$ has a unique structure of $\mathrm{dg}$ vertex Poisson algebra such that $a_{(n)} b=\delta_{n, 0}\{a, b\}_{R}$ for $a, b \in R \subset J_{\infty}(R)$. In particular, for a dg Lie algebra $\mathfrak{l}$, the arc space $J_{\infty}(\operatorname{Sym}(\mathfrak{l}))$ of the Kirillov-Kostant Poisson algebra (see the end of $§ 0.2 .1$ ) is a dg vertex Poisson algebra.

0.2.4. Derived gluing of vertex Poisson algebras. In $\S 5$, we introduce a vertex Poisson analogue of the derived gluing (0.12) and define the category $\mathrm{MT}_{\text {co }}$ of "coisson Moore-Tachikawa varieties".

Let $\mathfrak{l}$ be a dg Lie algebra. In the vertex Poisson world, the corresponding notion of momentum map is given by a morphism $\mu_{\mathrm{co}}: J_{\infty}(\operatorname{Sym}(\mathfrak{l})) \rightarrow P$ of dg vertex Poisson algebras, called a coisson momentum map. The coisson BRST complex is defined to be

$$
\operatorname{BRST}_{\mathrm{co}}\left(J_{\infty}(\mathfrak{l}), P, \mu_{\mathrm{co}}\right):=\left(P \otimes \mathrm{Cl}_{\mathrm{co}}(\mathfrak{l}), d_{\mathrm{co}}\right),
$$

where $\mathrm{Cl}_{\mathrm{co}}(\mathfrak{l})$ denotes the Clifford vertex Poisson algebra (Definition 4.3.11). See Definition 5.1.2 for the detail of th coisson BRST complex.

A vertex Poisson analogue of the gluing (0.12) is defined in the following way: Let $\mathfrak{g}_{1}, \mathfrak{g}_{2}$ and $\mathfrak{g}_{3}$ be the Lie algebras of semisimple Lie groups $G_{1}, G_{2}$ and $G_{3}$. Let also $\mu_{P}: J_{\infty}\left(\operatorname{Sym}\left(\mathfrak{g}_{1} \oplus \mathfrak{g}_{2}\right)\right) \rightarrow P, \mu_{P^{\prime}}$ : $J_{\infty}\left(\operatorname{Sym}\left(\mathfrak{g}_{2} \oplus \mathfrak{g}_{3}\right)\right) \rightarrow P^{\prime}$ be morphisms of dg vertex Poisson algebras. Then we define the coisson gluing to be

$$
P^{\prime} \widetilde{\circ} P:=\operatorname{BRST}_{\mathrm{co}}\left(J_{\infty}\left(\mathfrak{g}_{2}\right), P^{\mathrm{op}} \otimes P^{\prime}, \mu\right)
$$

Here $P^{\text {op }}$ denotes the opposite dg vertex Poisson algebra of $P$. See Definition 5.2.2 for the detail.

Using this operation, we introduce the category $\mathrm{MT}_{\text {со }}$ (Definition 5.2.3). An object is the same as that of the category MT, and a morphisms from $\mathfrak{g}_{1}$ to $\mathfrak{g}_{2}$ is a $\mathrm{dg}$ vertex Poisson algebra $P$ equipped with a morphism $J_{\infty}\left(\operatorname{Sym}\left(\mathfrak{g}_{1} \oplus \mathfrak{g}_{2}\right)\right) \rightarrow P$ and satisfying some finiteness condition. Composition of morphism is given by the coisson gluing.

For a dg vertex Poisson algebra $P$, we have a $\mathrm{dg}$ Poisson algebra $R_{P}^{\text {co }}:=P / \operatorname{Im}(T)$, where $T$ denotes the translation of $P$. See Definition 4.3 .3 for the detail. The main statement in $\S 5$ is that this construction induces a functor

$$
R_{(-)}^{\mathrm{co}}: \mathrm{MT}_{\text {co }} \longrightarrow \mathrm{MT}
$$

See Proposition 5.2.4 and Theorem 5.2.5 for the detail.

0.2.5. Derived gluing of $d g$ vertex algebras. In the final $\S 6$ we study the gluing for dg vertex algebras.

We denote by $V_{k}(\mathfrak{g})$ the universal affine vertex algebra at level $k$ for a finite dimensional semisimple Lie algebra $\mathfrak{g}$ as in $\S 0.1 .2$. In the vertex world, the corresponding notion of momentum map is a morphism $\mu_{V}: V_{k}(\mathfrak{g}) \rightarrow V$ of dg vertex algebras, called a chiral momentum map. See Remark 6.1.4 for the difference of the terminology of $[\mathrm{A}]$ and ours. Such a datum $\left(V, \mu_{V}\right)$ will be called a dg vertex algebra object in $V_{k}(\mathfrak{g})$-dgVMod. 
For a dg vertex algebra object $\left(V, \mu_{V}\right)$ in $V_{k}(\mathfrak{g})$-dgVMod, we have the BRST complex

$$
\operatorname{BRST}\left(\widehat{\mathfrak{g}}_{k}, V, \mu\right):=\left(V \otimes \bigwedge^{\frac{\infty}{2}}(\mathfrak{g}), d_{\mathrm{cl}}\right),
$$

where $\bigwedge^{\frac{\infty}{2}}(\mathfrak{g})$ denotes the free fermionic vertex algebra. See Definition 6.1.5 for the detail.

For another dg vertex algebra object $\left(V^{\prime}, \mu_{V^{\prime}}\right)$ in $V_{l}(\mathfrak{g})$-dgVMod, we define the chiral gluing $V^{\prime} \widetilde{\circ} \in \in$ $V_{k+l}(\mathfrak{g})$-dgVMod to be

$$
V^{\text {op }} \widetilde{\circ} V^{\prime}:=\operatorname{BRST}\left(\widehat{\mathfrak{g}}_{k+l}, V \otimes V^{\prime}, \mu\right)
$$

with $\mu: V^{\text {op }} \otimes V^{\prime}$ defined by $\mu(a, b):=-\mu_{V}(a)+\mu_{V^{\prime}}(b)$.

Using the chiral gluing, we introduce the category $\mathrm{MT}_{\mathrm{ch}}$ which is a vertex algebra analogue of the category MT. An object is the same as that of MT, and a morphisms from $\mathfrak{g}_{1}$ to $\mathfrak{g}_{2}$ is a dg vertex algebra $V$ equipped with a chiral momentum map $V_{k}\left(\mathfrak{g}_{1}\right) \otimes V_{l}\left(\mathfrak{g}_{2}\right) \rightarrow V$ and satisfying some finiteness condition. Composition of morphism is given by the chiral gluing. See Definition 6.3.2 for the precise description.

We then have a functor $R_{(-)}: \mathrm{MT}_{\mathrm{ch}} \rightarrow \mathrm{MT}$ induced by the procedure $V \mapsto R_{V}$ taking Zhu's $C_{2}$-algebra. We also have a functor $\operatorname{gr}^{F}: \mathrm{MT}_{\mathrm{ch}} \rightarrow \mathrm{MT}_{\text {co }}$ induced by the procedure $V \mapsto \mathrm{gr}^{F} V$ taking the associated graded space of the Li filtration. They sit in a commutative diagram

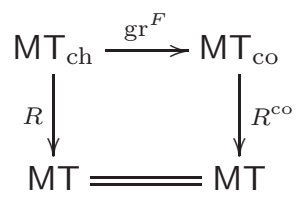

It would imply compatibility of gluing constructions in (0.9) (replacing VA by $\mathrm{MT}_{\text {ch }}$ and HS by MT). See Theorem 6.3.3 for the detail.

0.3. Global notation. Here is a list of global notations.

(1) $\delta_{m, n}$ denotes the Kronecker delta.

(2) The symbol $\mathbb{N}$ denotes the set of non-negative integers.

(3) The words ring and algebra mean associative ones unless otherwise stated.

(4) The word $d g$ means differential graded.

(5) On $\infty$-categories.

(i) We follow [Lu1] for the terminology on $\infty$-categories. In particular, an $\infty$-category is a simplicial set satisfying the weak Kan condition [Lu1, Definition 1.1.2.4].

(ii) For an $\infty$-category $\mathcal{C}$, we write $x \in \mathcal{C}$ to mean that $x$ is an object of $\mathcal{C}$, i.e., a vertex of the simplicial set $\mathcal{C}$.

(iii) The homotopy category [Lu1, $\$ 1.1 .4]$ of an $\infty$-category $\mathcal{C}$ is denoted by he.

(iv) The $\infty$-category $\mathcal{S}$ of spaces [Lu1, Definition 1.2.16.1] is defined to be the simplicial nerve of the simplicial category of Kan complexes. Its object will be called a space.

(v) We denote $\mathcal{H}:=\mathrm{h} \mathcal{S}$. We have the notion of homotopy group $\pi_{n} X$ for $X \in \mathcal{H}$.

(vi) For objects $x, y$ of an $\infty$-category $\mathcal{C}$, we denote by $\operatorname{Map}_{\mathcal{C}}(x, y) \in \mathcal{H}$ the mapping space from $x$ to $y$ [Lu1, Definition 1.2.2.1]. It is the homotopy type of the space representing the maps $x \rightarrow y$ in the homotopy category of the simplicial category attached to $\mathcal{C}$. We also denote $\operatorname{Hom}_{\mathcal{C}}(x, y):=\pi_{0} \operatorname{Map}_{\mathcal{C}}(x, y)$.

(vii) For an $\infty$-category $\mathcal{C}$ and $x, y, z \in \mathcal{C}$, we denote the pullback by $x \times_{y} z$ if they exist [Lu1, §4.4.2].

(viii) For an $\infty$-category $\mathcal{C}$, its opposite $\infty$-category [Lu1, $§ 1.2 .1]$ is denoted by $\mathcal{C}^{\text {op }}$.

(ix) An ordinary category is identified with its nerve and regarded as an $\infty$-category. We use sansserif font to denote ordinary categories such as the category dgVec of complexes.

(x) A dg category is identified with its differential graded nerve [Lu2, §1.3.1] and regarded as an $\infty$-category. We use bold letters to denote $\mathrm{dg}$ categories such as the $\mathrm{dg}$ category $\mathbf{d g V e c}$ of complexes.

Acknowledgements. The author thanks Tomoyuki Arakawa for his detailed explanation on $[\mathrm{A}]$ in the intensive course at Nagoya University in November, 2019. 


\section{Poisson algebras in DG Setting}

We work over a fixed field $\mathbb{k}$ of characteristics 0 .

1.1. Dg convention. For the definiteness, let us start with our dg convention.

1.1.1. Graded and $d g$ linear spaces. Let $\mathrm{Vec}$ be the category of linear spaces and linear maps over $\mathbb{k}$. We denote by $\operatorname{Hom}_{\mathbb{k}}(-,-)=\operatorname{Hom}_{V e c}(-,-)$ the linear space of linear maps, by $V^{*}:=\operatorname{Hom}_{\mathbb{k}}(V, \mathbb{k})$ the linear dual of $V \in \mathrm{Vec}$, and by $\otimes_{\mathbb{k}}$ the tensor product of linear spaces. The braiding (or the commutativity) isomorphism on the tensor product is an isomorphism $V \otimes W \stackrel{\sim}{\rightarrow} W \otimes V$ in Vec given by $v \otimes w \mapsto w \otimes v$. These give Vec a structure of $\mathbb{k}$-linear unital symmetric monoidal category with the unit $\mathbb{k}$.

Definition 1.1.1. (1) A graded linear space is a linear space equipped with an extra $\mathbb{Z}$-grading. We express the $\mathbb{Z}$-grading by superscript as $V^{\bullet}=\bigoplus_{n \in \mathbb{Z}} V^{n}$. An element $v \in V^{n}$ for some $n \in \mathbb{Z}$ will be called homogeneous, and for such an element we denote $|v|:=n$.

(2) A morphism of graded linear spaces is a homogeneous linear map of degree 0. For such a morphism $f: V \rightarrow W$, we denote $f^{n}:=\left.f\right|_{V^{n}}: V^{n} \rightarrow W^{n}$. We denote by gVec the category of graded linear space and their morphisms.

(3) For a graded linear space $V$, we denote $V[1]$ the graded linear space with $V[1]^{n}:=V^{n+1}$ for each $n \in \mathbb{Z}$

Definition 1.1.2. (1) A dg linear space or a complex is a pair $(V, d)$ consisting of a graded vector space $V$ and a morphism $d: V \rightarrow V[1]$ of graded vector spaces satisfying $d^{2}=0$. The morphism $d$ is called the differential, and the $\mathbb{Z}$-grading on $V$ is called the degree or the cohomological degree.

(2) A morphism of complexes is a morphism of graded linear spaces which respects the differentials. In particular, a morphism $f: V \rightarrow W$ of complexes preserves the $\mathbb{Z}$-grading: $f\left(V^{n}\right) \subset W^{n}$ for any $n \in \mathbb{Z}$.

(3) We denote by dgVec the category of complexes and their morphisms.

Remark. (1) We denote a complex simply by $V=(V, d)$ if no confusion may occur. We also denote $\left(V^{\bullet}, d_{V}\right)$ to emphasize the $\mathbb{Z}$-grading and that the differential is attached to $V$.

(2) As for the differential $d$ of a complex $V$, we have $d^{n}: V^{n} \rightarrow V^{n+1}$ and $d^{n+1} d^{n}=0$ using the notation in Definition 1.1.1 (2),

(3) Hereafter we regard a graded linear space as a complex with trivial differential. Thus we regard $\mathrm{gVec} \subset \mathrm{dg}$ Vec as a full subcategory.

A category enriched over dgVec will be called a $d g$ category. The complexes of morphisms in a dg category are called the hom complexes. A typical example of a dg category is the dg category of complexes. Let us recall the precise definition.

Definition 1.1.3. (1) For $V, W \in \operatorname{dgVec}$, we define $\underline{\operatorname{Hom}}(V, W)$ to be the complex of which

- the underlying graded linear space is given by

$$
\underline{\operatorname{Hom}}(V, W)=\bigoplus_{n \in \mathbb{Z}} \underline{\operatorname{Hom}}(V, W)^{n}, \quad \underline{\operatorname{Hom}}(V, W)^{n}:=\prod_{i \in \mathbb{Z}} \operatorname{Hom}_{\mathbb{k}}\left(V^{i}, W^{i+n}\right),
$$

- and the differential is given by $d f:=d_{W} \circ f-(-1)^{n} f \circ d_{V}$ for $f \in \underline{\operatorname{Hom}}(V, W)^{n}$.

We call $\underline{\operatorname{Hom}}(V, W)$ the internal hom in $\operatorname{dgVec}$. An element of $\underline{\operatorname{Hom}}(V, W)^{n}$ will be called a homogeneous linear map of cohomological degree $n$.

(2) We denote by $\mathbf{d g} \mathbf{V e c}$ the dg category of which the objects are complexes and the hom complexes are given by $\underline{\operatorname{Hom}}(-,-)$.

Let us recall the standard symmetric monoidal structure on dgVec.

Definition 1.1.4. Let $V, W \in \mathrm{dg} V e c$.

(1) The tensor product $V \otimes W$ in $\mathrm{dg} V e c$ is a complex of which

- the underlying linear space is $V \otimes_{\mathbb{k}} W$,

- the $\mathbb{Z}$-grading is given by

$$
(V \otimes W)^{n}:=\bigoplus_{r+s=n} V^{r} \otimes_{\mathbb{k}} W^{s},
$$

- and the differential is given by

$$
d_{V \otimes W}(v \otimes w):=d_{V} v \otimes w+(-1)^{|v|} v \otimes d_{W} w
$$

for any homogeneous $v \in V$ and any $w \in W$. 
(2) For homogeneous linear maps $f: V \rightarrow V^{\prime}$ and $g: W \rightarrow W^{\prime}$ of complexes, we define the linear map $f \otimes g: V \otimes W \rightarrow V^{\prime} \otimes W^{\prime}$ by

$$
(f \otimes g)(v \otimes w):=(-1)^{|g||v|} f(v) \otimes g(w)
$$

for homogeneous $v \in V$ and $w \in W$. This rule will be called the Koszul sign rule. In particular, we have a tensor product for a morphism of complexes.

(3) and the braiding isomorphism in dgVec is defined to be

$$
V \otimes W \stackrel{\sim}{\longrightarrow} W \otimes V, \quad v \otimes w \longmapsto(-1)^{|v||w|} w \otimes v
$$

for homogeneous $v \in V$ and $w \in W$.

These give the category $d g V e c$ a structure of unital symmetric monoidal category with the unit $\mathbb{k}$, which is denoted by $\mathrm{dgVec}^{\otimes}$. We also have the notion of a monoidal $d g$ category, and the dg category $\mathbf{d g V e c}$ of complexes has a structure of unital symmetric monoidal dg category, which is denoted by $\mathbf{d g V e c}{ }^{\otimes}$.

Let us recall the shift functor on $\mathrm{dgVec}$ :

Definition. (1) We denote by $\mathbb{k}[1]$ the complex whose underlying graded linear space is $(\mathbb{k}[1])^{n}=\delta_{n, 0} \mathbb{k}$ and whose differential is 0 .

(2) The shift functor [1] on dgVec is given by $V \mapsto V[1]:=\mathbb{k}[1] \otimes V$. In particular, we have $d_{V[1]}^{n}=-d_{V}^{n+1}$ for any $n \in \mathbb{Z}$. The inverse is denoted by $[-1]$, and for $m \in \mathbb{Z}$ the $m$-th repetition is denoted by $[m]$.

Here are some basic constructions of complexes.

Example 1.1.5. Let $V$ be a complex.

(1) We define the complex $T(V)$ by

$$
T(V):=\bigoplus_{p \in \mathbb{N}} V^{\otimes p}
$$

and call it the tensor space of $V$. In particular, the $\mathbb{Z}$-grading of $v_{1} \otimes \cdots \otimes v_{p} \in V^{\otimes p}$ is given by $\left|v_{1} \otimes \cdots \otimes v_{p}\right|:=\sum_{i=1}^{p}\left|v_{i}\right|$. We call the $\mathbb{N}$-grading given by the tensor power $p$ the weight.

(2) We define the symmetric tensor space $\operatorname{Sym}(V)$ of $V$ to be the complex

$$
\operatorname{Sym}(V):=\bigoplus_{p \in \mathbb{N}} V^{\otimes p} / \mathfrak{S}_{p}
$$

where the $p$-th symmetric group $\mathfrak{S}_{p}$ acts on $V^{\otimes p}$ by permutation. Similarly as in (1), we call the $\mathbb{N}$-grading given by the tensor power $p$ the weight.

For later reference, we give:

Example 1.1.6. (1) Let $\mathbb{k}[1]$ be the shifted one-dimensional linear space regarded as a complex with trivial differential. Consider the shifted symmetric tensor space $\operatorname{Sym}(\mathbb{k}[1])$. Its weight $n$ component is $(\mathbb{k}[1])^{\otimes n} / \mathfrak{S}_{n}$, where the $n$-th symmetric group $\mathfrak{S}_{n}$ acts by permutation. Using the braiding isomorphism in dgVec (Definition 1.1.4 (3)), we can identify

$$
(\mathbb{k}[1])^{\otimes n} \simeq \text { the signature representation }
$$

as $\mathfrak{S}_{n}$-representations. Thus $\operatorname{Sym}(\mathbb{k}[1])$ can be regarded as a sequence of signature representations of symmetric groups.

(2) Let $V$ be a complex and consider the shifted symmetric tensor space $\operatorname{Sym}(V[1])$. By the consideration in (1), we can identify

$$
(V[1])^{\otimes p} \simeq \bigwedge^{p}(V)
$$

as linear spaces, where the right hand side denotes the exterior product space. Abusing terminology, we call the complex $\operatorname{Sym}(V[1])$ the exterior product space of $V$.

We close this part by recalling the cohomology and the cone.

Definition 1.1.7. (1) The cohomology $H(V, d)$ of a complex $(V, d)$ is defined to be the graded linear space given by $H^{n}(V, d):=\operatorname{Ker}\left(d: V^{n} \rightarrow V^{n+1}\right) / \operatorname{Im}\left(d: V^{n-1} \rightarrow V^{n}\right)$. We denote $H^{\bullet}(V, d)$ to emphasize the $\mathbb{Z}$-grading. If confusion will not occur, we simply denote $H(V):=H(V, d)$.

(2) A complex $V$ is called acyclic if $H(V)=0$.

(3) For a morphism $f: V \rightarrow W$ in $\operatorname{dgVec}$, the induced morphism $H(V) \rightarrow H(W)$ in gVec is denoted by $H(f)$. Thus, we have a functor $H: \mathrm{dg} V e c \rightarrow \mathrm{gVec}$.

(4) A morphism $f: V \rightarrow W$ is called a quasi-isomorphism if the morphism $H(f): H(V) \rightarrow H(W)$ is an isomorphism in $\mathrm{gVec}$. 
Definition 1.1.8. For a morphism $f: V \rightarrow W$ of complexes, we define the mapping cone of $f$ to be the complex

$$
\text { Cone }(f):=V[1] \oplus W, \quad d_{\operatorname{Cone}(f)}:=\left[\begin{array}{cc}
d_{V[1]} & 0 \\
f[1] & d_{W}
\end{array}\right]
$$

Let us also recall the following standard fact:

Fact 1.1.9. Let $f: V \rightarrow W$ be a morphism in dgVec. Then $f$ is a quasi-isomorphism if and only if the mapping cone Cone $(f)$ is acyclic.

In particular, for a complex $V$, the mapping cone Cone $\left(\operatorname{id}_{V}\right)$ of the identity $\operatorname{id}_{V}: V \rightarrow V$ is acyclic.

1.2. Algebraic structures in dg setting. We collect here the terminology of algebraic structures in the $\mathrm{dg}$ setting. The most appropriate language here is the theory of operad, but we avoid to use it since we don't need such a full generality. We continue to work over a field $\mathbb{k}$ with characteristics 0 .

Recall the symmetric monoidal structures $\otimes$ on dgVec (Definition 1.1.4).

1.2.1. Dg algebras. We start with ring objects in dgVec.

Definition 1.2.1. (1) A dgalgebra ( $d g a$ for short) is a unital associative ring object in the monoidal category dgVec. In other words, it is a triple $(A, \cdot, u)$ consisting of

- a complex $A=\left(A, d_{A}\right)$,

- a morphism $\cdot: A \otimes A \rightarrow A$ in $\operatorname{dgVec}$ called the multiplication, and

- a morphism $u: \mathbb{k} \rightarrow A$ in dgVec called the unit (morphism)

satisfying the standard axioms of associativity and unitality. We often omit the symbol · of the multiplication and denote $a b:=a \cdot b$ for $a, b \in A$. We also denote the image of the unit $1_{\mathbb{k}}$ of the field $\mathbb{k}$ under the unit morphism by $1_{A}:=u\left(1_{\mathbb{k}}\right) \in A$ and call it the unit (element) of $A$.

(2) A morphism of dgas is a morphism in dgVec which respects the ring structures. We denote by dguAlg the category of dgas and their morphisms (the letter $\mathrm{u}$ indicates that we consider unital objects).

(3) The commutator on a dga $A$ is denoted by $[-,-]$. It is defined by

$$
[a, b]:=a b-(-1)^{|a||b|} b a
$$

for homogeneous elements $a, b \in A$ and is extended by linearity.

Remark. (1) We include unital condition in the definition.

(2) Let us recall a more down-to-earth definition of a dga. Let $A$ be a dga in the above sense. Since the multiplication : : $A \otimes A \rightarrow A$ is a morphism in dgVec, it commutes with the differentials $d_{A \otimes A}$ and $d_{A}$. Recalling the differential of the tensor product of complexes (Definition 1.1.4), we have $d_{A \otimes A}(a b)=\left(d_{A} a\right) \otimes b+(-1)^{|a|} a \otimes d_{A} b$ for $a, b \in A$. Then the commutativity of $\cdot$ and the differentials can be expressed as

$$
d_{A}(a b)=\left(d_{A} a\right) b+(-1)^{|a|} a\left(d_{A} b\right)
$$

This is nothing but the Leibniz rule, and the differential $d_{A}$ is a derivation with respect to the multiplication (see Definition 1.2.10 below).

Thus, we can restate the definition as: A dga is a triple $\left(A, \cdot, 1_{A}\right)$ consisting of

- a complex $A=\left(A, d_{A}\right)$,

- an associative multiplication $\cdot: A \times A \rightarrow A$ which preserves the $\mathbb{Z}$-grading and satisfies the Leibniz rule (1.1), and

- a unit element $1_{A} \in A^{0}$ with respect to the multiplication •.

Example 1.2.2. Let $V$ be a complex.

(1) The tensor algebra $T(V)$ is a typical example of a dga. The underlying complex is the tensor space (Example 1.1.5), and the multiplication is given by the concatenation of the tensor: $\left(v_{1} \otimes \cdots \otimes v_{p}\right)$. $\left(w_{1} \otimes \cdots \otimes w_{q}\right):=v_{1} \otimes \cdots \otimes v_{p} \otimes w_{1} \otimes \cdots \otimes w_{q}$.

(2) The endomorphism algebra End $(V)$ is another typical example. The underlying graded linear space is $\bigoplus_{n \in \mathbb{Z}} \operatorname{End}_{\mathbb{k}}(V)^{n}=\bigoplus_{n \in \mathbb{Z}} \overline{\operatorname{Hom}}_{\mathbb{k}}(V, V)^{n}$, the differential is $d_{\underline{\operatorname{End}}(V)} f=d_{V} \circ f-(-1)^{|f|} f \circ d_{V}$ (see Definition 1.1.3 (1)), and the multiplication is given by the composition. In particular, we have the commutator $[-,-]$ on $\underline{\operatorname{End}}(V)$.

Let us also recall the monoidal structure on dguAlg. 
Definition 1.2.3. The tensor product $A \otimes B$ of dgas $A$ and $B$ is defined to be the graded algebra whose underlying graded linear space is $A \otimes B \in \mathrm{dgVec}$ and whose multiplication is given by

$$
\left(a_{1} \otimes b_{1}\right) \cdot\left(a_{2} \otimes b_{2}\right):=(-1)^{\left|a_{2}\right|\left|b_{1}\right|} a_{1} a_{2} \otimes b_{1} b_{2}
$$

for homogeneous elements. The tensor product of morphisms of dgas are defined by the same formula.

This tensor product gives dguAlg a structure of a monoidal category.

We also have the notion of modules over a dga:

Definition. Let $A$ be a dga.

(1) A left $d g$ A-module $M$ is a complex equipped with a morphism . : $A \otimes M \rightarrow M$ in dgVec called the (left) action satisfying the standard axioms of associativity and unitality.

(2) A morphism $f: M \rightarrow N$ of left $\operatorname{dg} A$-modules $M$ and $N$ is a morphism in dgVec which respects the $A$-module structures.

(3) We denote the category of left $\operatorname{dg} A$-modules by $A$-dgMod.

Explicitly, a left $\operatorname{dg} A$-module $M$ is a complex equipped with a homogeneous bilinear map . : $A \times M \rightarrow M$ of cohomological degree 0 satisfying the Leibniz rule

$$
d_{M}(a . m)=d_{A}(a) \cdot m+(-1)^{|a|} a \cdot d_{M}(m)
$$

for $a \in A$ and homogeneous $m \in M$.

For left $\operatorname{dg} A$-modules $M$ and $N$, we define the complex $\underline{\operatorname{Hom}}_{A}(M, N)$ by

$\underline{\operatorname{Hom}}_{A}(M, N):=\bigoplus_{n \in \mathbb{Z}} \operatorname{Hom}_{A}(M, N[n])$,

$\operatorname{Hom}_{A}(M, N[n]):=\{f \in \operatorname{Hom}(M, N[n]) \mid f$ respects $\operatorname{dg} A$-module structures of $M$ and $N[n]\}$.

Thus $\underline{\operatorname{Hom}}_{A}(M, N)$ is a subcomplex of $\underline{\operatorname{Hom}}(M, N)=\bigoplus_{n \in \mathbb{Z}} \underline{\operatorname{Hom}}(M, N[n])$. Here we used the fact that the shift $M[n]$ is naturally a left $\operatorname{dg} A$-module. The complex $\underline{\operatorname{Hom}}_{A}(M, N)$ is naturally a left $\operatorname{dg} A$-module. Thus we have the following definition:

Definition 1.2.4. Let $A$ be a dga.

(1) For left $\operatorname{dg} A$-modules $M, N$, we call $\underline{\operatorname{Hom}}_{A}(M, N)$ the $d g$ A-module of morphisms of A-modules.

(2) We denote by $A$-dgMod the resulting dg category of left $\operatorname{dg} A$-modules.

We also have the notions of right $d g$ A-modules, $d g$ A-bimodules and left/right/both-side $d g$ ideals. The details are omitted.

1.2.2. Commutative $d g$ algebras. Next we introduce commutative algebra objects in dgVec.

Definition. (1) A commutative dga (cdga for short) is a dga whose commutator always vanishes.

(2) We denote by dguCom the subcategory of dguAlg spanned by cdgas (the letter u indicates that we consider unital objects).

Example 1.2.5. Let $V$ be a complex.

(1) Recall the symmetric tensor space $\operatorname{Sym}(V)$ (Example 1.1.5). It is a cdga with the multiplication induced by that on the tensor algebra $T(V)$ (Example 1.2.2 (1)). We denote the resulting cdga by the same symbol $\operatorname{Sym}(V)$ and call it the symmetric tensor algebra of $V$.

(2) The exterior product space $\operatorname{Sym}(V[1])$ (Example 1.1.6) is also a cdga with the multiplication induced by that on the tensor algebra $T(V)$. We denote the resulting cdga by the same symbol $\operatorname{Sym}(V[1])$ and call it the exterior algebra of $V$.

The category dguCom inherits the monoidal structure of dguAlg (Definition 1.2.3), and the braiding isomorphisms on dgVec (Definition 1.1.4) makes dguCom a symmetric monoidal category.

We also have the notion of modules over cdgas.

Definition. Let $A$ be a cdga.

(1) A $d g A$-module $M$ is a left $\operatorname{dg} A$-module where $A$ is regarded as a dga. We denote the category of dg $A$-modules by $A$-dgMod.

(2) The category $A$-dgMod has a structure of a symmetric monoidal category. We denote the tensor product by $\otimes_{A}$.

We also have the dg category of $\operatorname{dg} A$-modules, whose hom complex from $M$ to $N$ is defined to be the subcomplex $\underline{\operatorname{Hom}}_{A}(M, N) \subset \underline{\operatorname{Hom}}(M, N)$ of morphisms respecting the $\operatorname{dg} A$-module structures. 
Notation 1.2.6. For a cdga $A$, we denote by $A$-dgMod the $\operatorname{dg}$ category of $\operatorname{dg} A$-modules, and denote by $\underline{\operatorname{Hom}}_{A}(-,-)$ the hom complex in $A$-dgMod.

Let us introduce some dg modules over cdgas which will appear repeatedly in the following subsections.

Example 1.2.7. Let $A$ be a cdga and $M$ be a $\operatorname{dg} A$-module.

(1) We denote the iterated tensor products of $M$ as $M^{\otimes_{A} 2}:=M \otimes_{A} M, M^{\otimes_{A} 3}:=M \otimes_{A} M \otimes_{A} M$ and so on. We define the $\operatorname{dg} A$-module $T_{A}(M)$ by

$$
T_{A}(M):=\bigoplus_{p \in \mathbb{N}} M^{\otimes_{A} p}
$$

and call it the tensor algebra of $M$ over $A$.

(2) We define the $\operatorname{dg} A$-module $\operatorname{Sym}_{A}(M)$ by

$$
\operatorname{Sym}_{A}(M):=\bigoplus_{p \in \mathbb{N}} M^{\otimes}{ }_{A} p / \mathfrak{S}_{n},
$$

where the $p$-th symmetric group $\mathfrak{S}_{p}$ acts on $M^{\otimes_{A} p}$ by permutation. We call it the symmetric tensor algebra of $M$ over $A$.

These dg $A$-modules have the extra $\mathbb{N}$-grading given by the tensor power $p$. We call this extra grading the weight grading, following the terminology in Example 1.1.5.

1.2.3. Dg Lie algebras. We finally introduce Lie algebra objects.

Definition 1.2.8. (1) A graded Lie algebra is a Lie algebra object in the symmetric monoidal category gVec. In other words, it is a graded linear space $\mathfrak{l}$ together with a bilinear map [-.-] $: \mathfrak{l} \times \mathfrak{l} \rightarrow \mathfrak{l}$ called the Lie bracket satisfying

(i) $[x, y]=(-1)^{1+|x||y|}[y, x]$ for homogeneous $x, y \in \mathfrak{l}$, and

(ii) $(-1)^{|z||x|}[x,[y, z]]+(-1)^{|x||y|}[y,[z, x]]+(-1)^{|y||z|}[z,[x, y]]=0$ for homogeneous $a, b, c \in \mathfrak{l}$.

(2) A $d g$ Lie algebra is a Lie algebra object in the symmetric monoidal category dgVec. In other words, it is a triple $(\mathfrak{l}, d,[-,-])$ consisting of a graded linear space $\mathfrak{l}$, a morphism $d \in \operatorname{Hom}_{\mathrm{gVec}}(\mathfrak{l}, \mathfrak{l}[1])$, and a bilinear map [-.- ] : $\mathfrak{l} \times \mathfrak{l} \rightarrow \mathfrak{l}$ such that

(i) $(\mathfrak{l}, d)$ is a complex,

(ii) $(\mathfrak{l},[-,-])$ is a graded Lie algebra, and

(iii) $d[x, y]=[d x, y]+(-1)^{|x|}[x, d y]$ for homogeneous $x, y \in \mathfrak{l}$.

We have a natural notion of morphisms of $\mathrm{dg}$ Lie algebras, and denote by dgLie the category of $\mathrm{dg}$ Lie algebras and their morphisms.

Let us give an example of a dg Lie algebra arising from a dga. Recall that we impose

Example 1.2.9. Let $A=\left(A^{\bullet}, d_{A}, \cdot\right)$ be a dga, and $[-,-]$ be the commutator in $A$ (Definition 1.2.1). Then the triple $A_{L}:=\left(A^{\bullet}, d_{A},[-,-]\right)$ is a dg Lie algebra. The correspondence $A \mapsto A_{L}$ gives rise to a functor

$$
(-)_{L}: \text { dguAlg } \longrightarrow \text { dgLie. }
$$

In particular, the endomorphism algebra $\underline{\operatorname{End}}(V)$ of a complex $V$ (Example 1.2.2 (2)) has a structure of dg Lie algebra.

Let us now recall the notion of modules over a dg Lie algebra.

Definition. Let $\mathfrak{l}$ be a dg Lie algebra.

(1) A $d g \mathfrak{l}$-module is a complex $M$ equipped with a morphism $\rho: \mathfrak{l} \rightarrow \underline{\operatorname{End}}(M)$ of dg Lie algebras, where $\underline{\operatorname{End}}(M)$ is regarded as a dg Lie algebra by Example 1.2.9.

(2) A morphism of $\mathrm{dg} \mathfrak{l}$-modules is a morphism of complexes which respects the $\mathrm{dg} \mathfrak{l}$-module structures.

(3) The category of $\mathrm{dg} \mathfrak{l}$-modules is denoted by $\mathfrak{l}$-dgMod. It is naturally a symmetric monoidal category. The tensor product is denoted by $\otimes_{\mathfrak{r}}$.

For a $\operatorname{dg} \mathfrak{l}$-module $M$, the action of $x \in \mathfrak{l}$ on $m \in M$ is denoted by $x . m:=\rho(x)(m)$.

For the explanation of another example of a dga, we introduce:

Definition 1.2.10. Let $A$ be a dga and $M$ be a left $A$-module.

(1) Let $n \in \mathbb{Z}$. A derivation of degree $n$ from $A$ to $M$, or an $n$-derivation from $A$ to $M$, is a morphism $\theta \in \operatorname{Hom}_{\mathrm{dgVec}}(A, M[n])$ such that for any homogeneous $a, b \in A$ we have

$$
\theta(a b)=(-1)^{(|a|+n)|b|} b \cdot \theta(a)+(-1)^{n|a|} a \cdot \theta(b) .
$$


(2) For $n \in \mathbb{Z}$, we denote by $\operatorname{Der}(A, M)^{n}$ the set of $n$-derivations from $A$ to $M$, which is naturally a linear space. We define the subcomplex $\operatorname{Der}(A, M) \subset \underline{\operatorname{Hom}}(A, M)$ to be

$$
\operatorname{Der}(A, M):=\bigoplus_{n \in \mathbb{Z}} \operatorname{Der}(A, M)^{n} \text {. }
$$

The differential is given by $d_{\operatorname{Der}(A, M)} \theta=d_{\underline{\operatorname{Hom}}(A, M)} \theta=d_{M} \theta-(-1)^{|\theta|} \theta d_{A}$. Then $\operatorname{Der}(A, M)$ is naturally a left $\operatorname{dg} A$-module, and we call it the left $d g$ A-module of derivations.

(3) For $M=A$, we denote $\operatorname{Der}(A)^{n}:=\operatorname{Der}(A, A)^{n}$ and $\operatorname{Der}(A):=\operatorname{Der}(A, A)$. An element of $\operatorname{Der}(A)^{n}$ is called an $n$-derivation on $A$.

Remark. (1) In the item (2) we can check $d_{\underline{\operatorname{Hom}}(A, M)} \theta \in \operatorname{Der}(A, M)$ by

$$
\begin{aligned}
& \left(d_{\underline{\operatorname{Hom}}(A, M)} \theta\right)(a b)=d_{M} \theta(a b)-(-1)^{n} \theta\left(d_{A}(a b)\right) \\
& =d_{M}\left((-1)^{(|a|+n)|b|} b \cdot \theta(a)+(-1)^{n|a|} a \cdot \theta b\right)-(-1)^{n} \theta\left(\left(d_{A} a\right) b+(-1)^{|a|} a\left(d_{A} b\right)\right) \\
& =(-1)^{(|a|+n)|b|}\left(\left(d_{A} b\right) \cdot \theta(a)+(-1)^{|b|} b \cdot d_{M} \theta(a)\right)+(-1)^{n|a|}\left(\left(d_{A} a\right) \cdot \theta(b)+(-1)^{|a|} a \cdot d_{M} \theta(b)\right) \\
& -(-1)^{n}\left((-1)^{(|a|+n+1)|b|} b \cdot \theta\left(d_{A} a\right)+(-1)^{n(|a|+1)}\left(d_{A} a\right) \cdot \theta(b)\right) \\
& -(-1)^{|a|+n}\left((-1)^{(|a|+n)(|b|+1)}\left(d_{A} b\right) \cdot \theta(a)+(-1)^{n|a|} a \cdot\left(\theta d_{A} b\right)\right) \\
& =(-1)^{(|a|+n+1)|b|} b \cdot\left(d_{M} \theta(a)-(-1)^{n} \theta\left(d_{A} a\right)\right)+(-1)^{(n+1)|a|} a \cdot\left(d_{M} \theta(b)-(-1)^{n} \theta\left(d_{A} b\right)\right) \\
& =(-1)^{(|a|+n+1)|b|} b \cdot\left(d_{\underline{\operatorname{Hom}}(A, M)} \theta\right)(a)+(-1)^{(n+1)|a|} a \cdot\left(d_{\underline{\operatorname{Hom}}(A, M)} \theta\right)(b)
\end{aligned}
$$

for $\theta \in \operatorname{Der}(A, M)^{n}$ and homogeneous $a, b \in A$.

(2) For a cdga $A$, we can rephrase the definition in (3) as follows. An $n$-derivation $\theta$ on $A$ is a homogeneous linear endomorphism on $A$ of degree $n$ such that $[\theta, a]=\theta(a)$ in the endomorphism algebra End $(A)$ (Example 1.2.2 (2)). Here in the left hand side we regard $a \in A \subset \underline{\operatorname{End}}(A)$ as a multiplication operator from left.

Now we can explain another example of a dg Lie algebra.

Example 1.2.11. For a cdga $A$, consider the $\operatorname{dg} A$-module $\operatorname{Der}(A)$ of derivations on $A$ (Definition 1.2.10 (3)). Also recall the dg Lie algebra $\operatorname{End}(A)$, where we regard $A$ as a complex (Example 1.2.9). Since we have $\operatorname{Der}(A) \subset \underline{\operatorname{End}}(A)$ as complexes, we can consider the commutator $[\theta, \varphi]$ for $\theta, \varphi \in \operatorname{Der}(A)$. Then we can check that $[\theta, \varphi] \in \operatorname{Der}(A)$, so that the triple $\left(\operatorname{Der}(A), d_{\operatorname{Der}(A)},[-,-]\right)$ is a dg Lie algebra. For later citation, we denote

and call $\mathbb{T}_{A}$ the $d g$ Lie algebra of derivations on $A$.

$$
\mathbb{T}_{A}:=\left(\operatorname{Der}(A), d_{\operatorname{Der}(A)},[-,-]\right)
$$

1.2.4. Chevalley-Eilenberg complex. In this subsection we recall the Chevalley-Eilenberg complex associated to a dg Lie algebra and its dg module. The material is more or less standard. We borrow some notations from $[\mathrm{BD}, 1.4 .5]$. Let us fix a dg Lie algebra $\mathfrak{l}=\left(\mathfrak{l}, d_{\mathfrak{l}},[-,-]_{\mathfrak{l}}\right)$ in this part.

Recall the mapping cone of a morphism of complexes (Definition 1.1.7). We denote by $\mathfrak{l}_{\uparrow}$ the mapping cone of the identify id ${ }_{\mathfrak{l}}: \mathfrak{l} \rightarrow \mathfrak{l}$. Thus, it is a complex given by

$$
\mathfrak{l}_{\dagger}:=\operatorname{Cone}\left(\mathrm{id}_{\mathfrak{l}}\right)=\mathfrak{l}[1] \oplus \mathfrak{l}, \quad d=\left[\begin{array}{cc}
d_{\mathfrak{l}[1]} & 0 \\
\operatorname{id}_{\mathfrak{l}}[1] & d_{\mathfrak{l}}
\end{array}\right] .
$$

It is acyclic by Fact 1.1.9.

We denote an element of $\mathfrak{l}_{\dagger}^{n}=\mathfrak{l}^{n+1} \oplus \mathfrak{l}^{n}$ as $(x, y)$ with $x \in \mathfrak{l}^{n+1}$ and $y \in \mathfrak{l}^{n}$. Then we have

$$
d^{n}(x, y)=\left(-d_{\mathfrak{l}}^{n+1} x, x+d_{\mathfrak{l}}^{n} y\right) \in \mathfrak{l}^{n+2} \oplus \mathfrak{l}^{n+1}=\mathfrak{l}_{\dagger}^{n+1} .
$$

Using the same notation, we can endow $\mathfrak{l}_{\dagger}$ with a dg Lie algebra structure by

$$
\left[(x, y),\left(x^{\prime}, y^{\prime}\right)\right]:=\left(\left[x, y^{\prime}\right]_{\mathfrak{r}}+\left[y, x^{\prime}\right]_{\mathfrak{r}},\left[y, y^{\prime}\right]_{\mathfrak{l}}\right) .
$$

We have an injective morphism of dg Lie algebras

$$
\mathfrak{l} \longleftrightarrow \mathfrak{l}_{\dagger}, \quad y \longmapsto(0, y) .
$$

By this injection, the mapping cone $\mathfrak{l}_{\dagger}$ is also a dg $\mathfrak{l}$-module.

Next recall the enveloping algebra $U(\mathfrak{l})$. It is the quotient dga $T(\mathfrak{l}) / I$ of the tensor algebra $T(\mathfrak{l})$ (Example 1.2.2 (1)) by the both-side dg ideal $I$ generated by terms of the form $x \otimes y-(-1)^{|x||y|}-[x, y]$ with $x, y \in \mathfrak{l}$. The correspondence $\mathfrak{l} \mapsto U(\mathfrak{l})$ gives a functor

$$
U: \text { dgLie } \longrightarrow \text { dguAlg, }
$$


which is left adjoint to the functor $(-)_{L}:$ dguAlg $\rightarrow$ dgLie in Example 1.2.9. Thus we have an adjunction $U:$ dgLie $\rightleftarrows$ dguAlg : $(-)_{L}$ of functors of categories. Let us also recall that there is a functorial equivalence

$$
\mathfrak{l} \text {-dgMod } \stackrel{\sim}{\longrightarrow} U(\mathfrak{l}) \text {-dgMod }
$$

between the category of $\mathrm{dg} \mathfrak{l}$-modules and that of $\operatorname{dg} U(\mathfrak{l})$-modules. Hereafter we identify these categories.

In particular, the universal enveloping algebra $U\left(\mathfrak{l}_{\dagger}\right)$ of the dg Lie algebra $\mathfrak{l}_{\dagger}$ is a dga, and it is also a dg $U(\mathfrak{l})$-module since $\mathfrak{l}_{\dagger}$ is a dg $\mathfrak{l}$-module. Then we can obtain the following statements by direct calculations.

Lemma 1.2.12. Let $\mathfrak{l}$ be a dg Lie algebra.

(1) As a graded linear space, we have $U\left(\mathfrak{l}_{\uparrow}\right) \simeq \operatorname{Sym}(\mathfrak{r}[1]) \otimes U(\mathfrak{l})$.

(2) Under the isomorphism $\operatorname{Sym}(\mathfrak{l}[1]) \simeq \Lambda(\mathfrak{l})$, the differential $d_{U\left(\mathfrak{l}_{+}\right)}$is given by

$$
\begin{aligned}
d_{U\left(\mathfrak{l}_{+}\right)}\left(x_{1} \wedge \cdots \wedge x_{p} \otimes u\right) & =\sum_{i=1}^{p}(-1)^{\sum_{a=1}^{i-1}\left(\left|x_{p}\right|+1\right)} x_{1} \wedge \cdots \wedge d_{\mathfrak{r}} x_{i} \wedge \cdots \wedge x_{n} \otimes u \\
& +\sum_{1 \leq i<j \leq p}(-1)^{\left(\left|x_{i}\right|+1\right) \sum_{a=i+1}^{j-1}\left(\left|x_{a}\right|+1\right)} x_{1} \wedge \cdots \widehat{x_{i}} \cdots \wedge\left[x_{i}, x_{j}\right] \wedge \cdots \wedge x_{n} \otimes u \\
& +\sum_{i=1}^{p}(-1)^{\left(\left|x_{i}\right|+1\right) \sum_{a=i+1}^{p}\left(\left|x_{a}\right|+1\right)} x_{1} \wedge \cdots \widehat{x_{i}} \cdots \wedge x_{n} \otimes x_{i} u \\
& +(-1)^{\sum_{a=1}^{p}\left(\left|x_{a}\right|+1\right)} x_{1} \wedge \cdots \wedge x_{n} \otimes d_{U(\mathfrak{l})} u .
\end{aligned}
$$

for $x_{i} \in \mathfrak{l}$ and $u \in U(\mathfrak{l})$.

Finally, let us recall the $\operatorname{dg} A$-module $\underline{\operatorname{Hom}}_{A}(-,-)$ of morphisms for a dga $A$ (Definition 1.2.4).

Definition 1.2.13. Let $\mathfrak{l}$ be a dg Lie algebra.

(1) For a $\mathrm{dg} \mathfrak{l}$-module $M$, we define the Chevalley-Eilenberg (cochain) complex $\operatorname{CE}(\mathfrak{l}, M)$ to be the $\mathrm{dg}$ l-module

The differential is denoted by $d_{\mathrm{CE}}$.

$$
\mathrm{CE}(\mathfrak{l}, M):=\underline{\operatorname{Hom}}_{U(\mathfrak{l})}\left(U\left(\mathfrak{l}_{\uparrow}\right), M\right) .
$$

(2) We denote the functor $M \mapsto \mathrm{CE}(\mathfrak{l}, M)$ by

$$
\mathrm{CE}(\mathfrak{l},-): \mathfrak{l} \text {-dgMod } \longrightarrow \mathfrak{l} \text {-dgMod. }
$$

Remark 1.2.14. (1) By Lemma 1.2.12 (1), we have

$$
\mathrm{CE}(\mathfrak{l}, M) \simeq \underline{\operatorname{Hom}}(\operatorname{Sym}(\mathfrak{r}[1]), M)
$$

as a graded linear space. Thus, we have $\mathrm{CE}(\mathfrak{l}, M) \simeq \operatorname{Hom}(\bigwedge \mathfrak{l}, M)$ as a linear space, which is the standard definition in the literature.

(2) By definition, the differential $d_{\mathrm{CE}}$ is given by

$$
d_{\mathrm{CE}} f=d_{M} f-(-1)^{|f|} f d_{U\left(\mathfrak{l}_{\dagger}\right)}
$$

for a homogeneous element $f \in \operatorname{Hom}(\bigwedge \mathfrak{l}, M)$ of cohomological degree $|f|$. Denoting the weight decomposition by $f=\sum_{n \in \mathbb{N}} f_{p}, f_{p} \in \operatorname{Hom}\left(\bigwedge^{p} \mathfrak{l}, M\right)$ and using the description $\operatorname{CE}(\mathfrak{l}, M) \simeq \operatorname{Hom}(\bigwedge \mathfrak{l}, M)$ in (1), we can write down $d_{\mathrm{CE}} f=\sum_{p \in \mathbb{N}}\left(d_{\mathrm{CE}} f\right)_{p}$ as:

$$
\begin{aligned}
\left(d_{\mathrm{CE}} f\right)_{p}\left(x_{1} \wedge \cdots \wedge x_{p}\right) & =d_{M} f_{p}\left(x_{1} \wedge \cdots \wedge x_{p}\right) \\
& +\sum_{i=1}^{p}(-1)^{|f|+\sum_{a=1}^{i-1}\left(\left|x_{a}\right|+1\right)} f_{p}\left(x_{1} \wedge \cdots \wedge\left(d_{\mathfrak{r}} x_{i}\right) \wedge \cdots \wedge x_{p}\right) \\
& +\sum_{1 \leq i<j \leq p}(-1)^{|f|+\left(\left|x_{i}\right|+\left|x_{j}\right|\right) \sum_{a=1}^{i-1}\left(\left|x_{a}\right|+1\right)+\left(\left|x_{j}\right|+1\right) \sum_{a=i+1}^{j-1}\left(\left|x_{a}\right|+1\right)} \\
& \quad f_{p-1}\left(\left[x_{i}, x_{j}\right] \wedge x_{1} \wedge \cdots \widehat{x_{i}} \cdots \widehat{x_{j}} \cdots \wedge x_{p}\right) \\
& +\sum_{i=1}^{p}(-1)^{\left(\left|x_{i}\right|+1\right)\left(|f|+\sum_{a=1}^{i-1}\left(\left|x_{a}\right|+1\right)\right)} x_{i} \cdot f_{p-1}\left(x_{1} \wedge \cdots \widehat{x_{i}} \cdots \wedge x_{p}\right) .
\end{aligned}
$$

In the non-dg case $|f|=\left|x_{i}\right|=0$ and $d_{\mathfrak{l}}=d_{M}=0$, we recover the original Chevalley-Eilenberg differential.

(3) If $\mathfrak{l}$ is of finite dimension, then we have an isomorphism

$$
\mathrm{CE}(\mathfrak{l}, M) \simeq \operatorname{Sym}\left(\mathfrak{l}^{*}[-1]\right) \otimes M
$$

as a graded linear space. Let us rewrite the differential $d_{\mathrm{CE}}$ under this isomorphism. Note that the graded Lie algebra $\mathfrak{l}_{\dagger}$ (forget the differential) acts on $\operatorname{Sym}\left(\mathfrak{l}^{*}[-1]\right)$ in the way that $\mathfrak{l} \subset \mathfrak{l}_{\dagger}$ acts by the coadjoint action and $\mathfrak{l}[1] \subset \mathfrak{l}_{\dagger}$ acts by $-\{\cdot, \cdot\}$, where $\{\cdot, \cdot\}: \mathfrak{l}^{*} \otimes \mathfrak{l} \rightarrow \mathbb{k}$ denotes the canonical pairing. Then there exits a unique differential $\delta_{\mathrm{CE}}$ on $\operatorname{Sym}\left(\mathfrak{l}^{*}[-1]\right)$ such that the $\mathfrak{l}_{+}$-action is compatible. Explicitly, $\left.\delta_{\mathrm{CE}}\right|_{\mathfrak{l}^{*}[-1]}$ is equal to the composition $\mathfrak{I}^{*}[-2] \stackrel{\nu}{\rightarrow} \mathfrak{l}^{*}[-1] \otimes \mathfrak{l}^{*}[-1] \stackrel{-\frac{1}{2} \cdot}{\longrightarrow} \operatorname{Sym}^{2}\left(\mathfrak{l}^{*}[-1]\right)$, where 
$\nu$ denotes the dual of the Lie bracket $[\cdot, \cdot]_{\mathrm{r}}$. Finally, $d_{\mathrm{CE}}$ is equivalent to $\delta_{\mathrm{CE}} \otimes \mathrm{id}+f+\mathrm{id} \otimes d_{M}$, where $f: M \rightarrow \mathfrak{l}^{*} \otimes M$ denotes the dual of the l-action.

The Chevalley-Eilenberg complex has an extra structure. For the explanation, we need:

Definition 1.2.15. Let $p, q \in \mathbb{N}$ and consider the symmetric group $\mathfrak{S}_{p+q}$. We denote by $\mathfrak{S}_{p, q} \subset \mathfrak{S}_{p+q}$ the subset of $(p, q)$-shuffles, i.e., permutations $\sigma$ such that $\sigma(1)<\cdots<\sigma(p)$ and $\sigma(p+1)<\cdots<\sigma(p+q)$.

Definition 1.2.16. Let $\mathfrak{l}$ be a dg Lie algebra.

(1) For $\operatorname{dg} \mathfrak{l}$-modules $M, N$, we define the morphism $\cup: \mathrm{CE}(\mathfrak{l}, M) \otimes \mathrm{CE}(\mathfrak{l}, N) \rightarrow \mathrm{CE}(\mathfrak{l}, M \otimes N)$ of $\operatorname{dg}$ l-modules by

$$
(f \cup g)\left(x_{1} \wedge \cdots \wedge x_{p+q}\right):=\sum_{\sigma \in \mathfrak{S}_{p, q}} \operatorname{sgn}(\sigma)(-1)^{\varepsilon+\varepsilon_{1}} f\left(x_{\sigma(1)} \wedge \cdots \wedge x_{\sigma(p)}\right) \otimes g\left(x_{\sigma(p+1)} \wedge \cdots \wedge x_{\sigma(p+q)}\right),
$$

for $f \in \mathrm{CE}(\mathfrak{l}, M)(m)$ and $g \in \mathrm{CE}(\mathfrak{l}, N)(n)$. Here $\varepsilon$ denotes the sign of the braiding isomorphisms for the permutation $\sigma$ of $x_{i}$ 's, and $\varepsilon_{1}:=p|g|+\sum_{i=1}^{p}\left|x_{\sigma(i)}\right|(q+|g|)$. We call the operation $\cup$ the cup product.

(2) Let $A$ be a commutative ring object in $\mathfrak{l}$-dgMod, i.e., a cdga which is also a dg l-module and the multiplication is a morphism in $\mathfrak{l}$-dgMod. Consider the composition

$$
\mathrm{CE}(\mathfrak{l}, A) \otimes \mathrm{CE}(\mathfrak{l}, A) \stackrel{\cup}{\longrightarrow} \mathrm{CE}(\mathfrak{l}, A \otimes A) \stackrel{\mathrm{CE}(\mathfrak{l}, \cdot)}{\longrightarrow} \mathrm{CE}(\mathfrak{l}, A),
$$

where the first $\cup$ denotes the cup product and the second $\operatorname{CE}(\mathfrak{l}, \cdot)$ is the image of the multiplication of $A$ under the functor $\mathrm{CE}(\mathfrak{l},-)$ (Definition 1.2.13). We denote this composition by the same symbol as

$$
\cup: \mathrm{CE}(\mathfrak{l}, A) \otimes \mathrm{CE}(\mathfrak{l}, A) \longrightarrow \mathrm{CE}(\mathfrak{l}, A) .
$$

Now we can check the following classical result.

Lemma 1.2.17. Let $A$ be a commutative ring object in $\mathfrak{l}$-dgMod. Then the Chevalley-Eilenberg complex $\mathrm{CE}(\mathfrak{l}, A)$ with the cup product $\cup: \mathrm{CE}(\mathfrak{l}, A) \otimes \mathrm{CE}(\mathfrak{l}, A) \rightarrow \mathrm{CE}(\mathfrak{l}, A)$ is a cdga. We call it the Chevalley-Eilenberg cdga.

1.3. Shifted Poisson algebras. In this subsection we recollect basics on shifted Poisson structures following [CPT+, M16, S17]. We continue to work over a field $\mathbb{k}$ with characteristics 0 .

\subsubsection{Definition.}

Definition 1.3.1. Let $n \in \mathbb{Z}$. A $\mathbb{P}_{n}$-algebra in $\mathrm{dgVec}$ is a data $(R, \cdot,\{-,-\})$ consisting of

- a cdga $(R, \cdot)$ and

- a morphism $\{-,-\}: R \otimes R \longrightarrow R[1-n]$ in $\mathrm{dgVec}$, called the $n$-Poisson bracket of $R$ which satisfies the following conditions.

(i) $\{-,-\}$ gives a structure of dg Lie algebra on $R[n-1]$ (Definition 1.2.8).

(ii) The Leibniz rule

$$
\{f, g \cdot h\}=\{f, g\} \cdot h+(-1)^{|g||h|}\{f, h\} \cdot g
$$

holds for homogeneous $f, g, h \in R$.

We often omit to mention the category $\mathrm{dgVec}$ and just call it a $\mathbb{P}_{n}$-algebra. We also call it a $d g n$-Poisson algebra. In the case $n=1$, we just call it a $d g$ Poisson algebra.

Remark 1.3.2. (1) If we replace $d g \bigvee e c$ by $\vee e c$, then a $\mathbb{P}_{1}$-algebra in $\vee e c$ is nothing but a (commutative) Poisson algebra in the ordinary sense.

(2) A $\mathbb{P}_{2}$-algebra (in $\mathrm{gVec}$ ) is nothing but a Gerstenhaber algebra. See [LV, 13.3.10-13.3.15] for the detail.

(3) The symbol $\mathbb{P}_{n}$ indicates that we can give a definition by a dg operad on the symmetric monoidal $\mathrm{dg}$ category dgVec of complexes. We refer [M16] for the detail.

Hereafter we often omit the symbol $\cdot$ and denote $r s:=r \cdot s$, and also denote just by $R$ the Poisson algebra $(R, \cdot,\{-,-\})$.

Let us also introduce the category of $\mathbb{P}_{n}$-algebras.

Definition. (1) A morphism of $\mathbb{P}_{n}$-algebras is defined to be a morphism in dguCom which respects the Poisson brackets.

(2) We define the tensor product of $\mathbb{P}_{n}$-algebras $\left(R,\{-,-\}_{R}\right)$ and $\left(S,\{-,-\}_{S}\right)$ to be the one where 
- the underlying cdga is the tensor product $R \otimes S$ in dguCom, and

- the Poison bracket $\{-,-\}$ is given by

$$
\left\{r \otimes s, r^{\prime} \otimes s^{\prime}\right\}:=\left\{r, r^{\prime}\right\}_{R} \otimes\left(s s^{\prime}\right)+(-1)^{\left|r^{\prime}\right||s|}\left(r r^{\prime}\right) \otimes\left\{s, s^{\prime}\right\}_{S}
$$

for homogeneous $r, r^{\prime} \in R$ and $s, s^{\prime} \in S$.

(3) We denote by $\mathbb{P}_{n}$-dgVec the category of $\mathbb{P}_{n}$-algebras and their morphisms. It is a symmetric monoidal category with respect to the tensor product. In the case $n=1$, we also denote $\operatorname{dgPA}:=\mathbb{P}_{1}$-dgVec.

Next we introduce notations for Poisson modules over $\mathbb{P}_{n}$-algebras.

Definition 1.3.3. (1) Let $R$ be a $\mathbb{P}_{n}$-algebra. A $d g$ Poisson $R$-module $M$ is a complex $M$ equipped with two morphisms

$$
\therefore: R \otimes M \longrightarrow M, \quad\{-,-\}: R \otimes M \longrightarrow M
$$

in $d g V e c$ such that

(i) the morphism . is a dg $R$-module structure where we regard $R \in$ dguCom,

(ii) $\{-,-\}$ is an $R[n-1]$-module structure where we regard $R[n-1] \in \mathrm{dgLie}$, and

(iii) the Leibniz rule

$$
\{r, s . m\}=\{r, s\} \cdot m+(-1)^{|r||s|} s \cdot\{r, m\}, \quad\{r \cdot s, m\}=r \cdot\{s, m\}+(-1)^{|r||s|} s \cdot\{r, m\}
$$

hold for any homogeneous $r, s \in R$ and $m \in M$.

We denote by $R$-dgPMod the category of dg Poisson $R$-modules.

(2) For a $\mathbb{P}_{1}$-Poisson algebra $R$ in Vec, i.e., a Poisson algebra in the ordinary sense, we define a Poisson $R$-module to be a linear space $M$ equipped with . and $\{-,-\}$ with the same conditions as in (1). We denote by $R$-PMod the category of Poisson $R$-modules.

1.3.2. Shifted polyvectors. Let us explain the space of shifted polyvectors with the Schouten bracket, which is a standard example of a shifted Poisson algebra. See [LPV, 3.3.2] for the non-dg case. We need some preliminaries.

Let $A$ be a cdga. For a dg $A$-module $M$, we have the $\operatorname{dg} A$-module $\operatorname{Der}(A, M)$ of derivations (Definition 1.2.10 (2)). The functor

$$
\operatorname{Der}(A,-): A \text {-dgMod } \longrightarrow A \text {-dgMod, } \quad M \longmapsto \operatorname{Der}(A, M)
$$

commutes with limits so that it is corepresentable by a $\operatorname{dg} A$-module.

Notation 1.3.4. Let $A$ be a cdga. We denote the $\operatorname{dg} A$-module corepresenting the functor $\operatorname{Der}(A,-)$ by $\Omega_{A}^{1}$, and call it the module of Kähler differentials over $A$.

We have an explicit description of $\Omega_{A}^{1}$. It is a $\operatorname{dg} A$-module

- generated over $A$ by the symbols $d a$ for each $a \in A$ with cohomological degree $|d a|:=|a|$, and

- the defining relation is

$$
d(a b)=(-1)^{|a||b|} b \cdot(d a)+(-1)^{|a|} a \cdot(d b) .
$$

Let us give a simple example for later citation.

Example 1.3.5. Let $V$ be a complex, and $\operatorname{Sym}(V)$ be the symmetric tensor algebra (Example 1.2.5 (1)). Then the module $\Omega_{\operatorname{Sym}(V)}^{1}$ of Kähler differentials is a free $\operatorname{dg} \operatorname{Sym}(V)$-module generated by $V$.

The universality of corepresenting object says that for a $\operatorname{dg} A$-module $M$ we have a functorial isomorphism

$$
\underline{\operatorname{Hom}}_{A}\left(\Omega_{A}^{1}, M\right) \stackrel{\sim}{\longrightarrow} \operatorname{Der}(A, M) .
$$

In particular, there is a morphism $d: A \rightarrow \Omega_{A}^{1}$ in $A$-dgMod, and the above functorial isomorphism is given by $\alpha \mapsto \alpha \circ d$. The morphism $d: A \rightarrow \Omega_{A}^{1}$ is nothing but the correspondence $a \mapsto d a$.

We need one more terminology.

Definition 1.3.6. A graded dg linear space, or a graded complex $M$ is a complex with an extra $\mathbb{Z}$-grading. We call the extra $\mathbb{Z}$-grading the weight of $M$ and denote by $M=\bigoplus_{p \in \mathbb{Z}} M(p)$. We denote the cohomological grading by $M(p)=\bigoplus_{n \in \mathbb{Z}} M(p)^{n}$. Thus we have

$$
M=\bigoplus_{p \in \mathbb{Z}} M(p)=\bigoplus_{p \in \mathbb{Z}} \bigoplus_{n \in \mathbb{Z}} M(p)^{n} .
$$

Example. We have already introduced complexes with extra $\mathbb{N}$-gradings. 
(1) Let $V$ be a complex. Then the tensor space (algebra) $T(V)$ and the symmetric tensor space (algebra) $\operatorname{Sym}(V)$ in Example 1.1.5 are graded complexes. Explicitly, we have

$$
T(V)(p)=V^{\otimes p}, \quad \operatorname{Sym}(V)(p)=V^{\otimes p} / \mathfrak{S}_{p} \quad(p \in \mathbb{N}) .
$$

(2) Let $A$ be a cdga and $M$ be a dg $A$-module. Then the tensor algebra $T_{A}(M)$ and the symmetric tensor algebra $\operatorname{Sym}_{A}(M)$ in Example 1.2.7 are graded complexes. Explicitly, we have

$$
T_{A}(M)(p)=M^{\otimes_{A} p}, \quad \operatorname{Sym}_{A}(M)(p)=M^{\otimes_{A} p} / \mathfrak{S}_{p} \quad(p \in \mathbb{N}) .
$$

Now we can introduce shifted polyvector fields over a cdga. Recall the hom complex $\underline{\operatorname{Hom}}_{A}(M, N) \in$ $A$-dgMod for $\operatorname{dg} A$-modules $M$ and $N$ (Notation 1.2.6).

Definition. Let $n \in \mathbb{Z}$ and $A$ be a cdga. We define the graded $\operatorname{complex} \operatorname{Pol}(A, n)$ of $n$-shifted polyvector fields by

$$
\operatorname{Pol}(A, n):=\underline{\operatorname{Hom}}_{A}\left(\operatorname{Sym}_{A}\left(\Omega_{A}^{1}[n+1]\right), A\right)
$$

with the weight grading $\operatorname{Pol}(A, n)(p):=\underline{\operatorname{Hom}}_{A}\left(\operatorname{Sym}_{A}\left(\Omega_{A}^{1}[n+1]\right)(p), A\right)$. An element of $\operatorname{Pol}(A, n)$ will be called a polyvector.

Let us explain the Poisson algebra structure on $\operatorname{Pol}(A, n)$. For that, we use the following notation in [S17]:

Notation 1.3.7. Let $n$ and $A$ be as above. We use the formal symbol $d_{\mathrm{dR}}$ sitting in the cohomological degree $-(n+1)$ in $\Omega_{A}^{1}[n+1]$ and denote

$$
d_{\mathrm{dR}} a \in\left(\Omega_{A}^{1}[n+1]\right)^{m-n-1}=\left(\Omega_{A}^{1}\right)^{m}
$$

for $a \in A^{m}$. We also denote by $\lrcorner: \operatorname{Pol}(A, n) \otimes \operatorname{Sym}_{A}\left(\Omega^{1}[n+1]\right) \rightarrow A$ the natural pairing. Then, for $v \in \operatorname{Pol}(A, n)(p)$ and $a_{i} \in A(i=1, \ldots, p)$, we set

$$
\left.v\left(a_{1}, \ldots, a_{p}\right):=v\right\lrcorner\left(d_{\mathrm{dR}} \otimes \cdots \otimes d_{\mathrm{dR}}\right)\left(a_{1} \otimes \cdots \otimes a_{p}\right) .
$$

Here the term $\left(d_{\mathrm{dR}} \otimes \cdots \otimes d_{\mathrm{dR}}\right)\left(a_{1} \otimes \cdots \otimes a_{p}\right)$ is regarded as a monomial in the $\operatorname{cdga} \operatorname{Sym}_{A}^{p}\left(\Omega_{A}^{1}[n+1]\right)$.

In (1.2), the sign rule for multiplication is given by Definition 1.2.3, and that for commutation relation is given by Definition 1.1.4. For example, using $n^{2} \equiv n(\bmod 2)$, we have

$$
v\left(a_{1}, a_{2}, a_{3}, \ldots, a_{p}\right)=(-1)^{\left|a_{1}\right|\left|a_{2}\right|+n+1} v\left(a_{2}, a_{1}, a_{3}, \ldots, a_{p}\right) .
$$

Fact 1.3.8 ([S17, $\S \S 1.1-1.2])$. Let $n \in \mathbb{Z}$ and $A$ be a cdga.

(1) For $v \in \operatorname{Pol}(A, n)(p)$ and $w \in \operatorname{Pol}(A, n)(q)$, the following formula defines $v \cdot w \in \operatorname{Pol}(A, n)(p+q)$.

$$
(v \cdot w)\left(a_{1}, \ldots, a_{p+q}\right):=\sum_{\sigma \in \mathfrak{S}_{p, q}} \operatorname{sgn}(\sigma)^{n}(-1)^{\varepsilon+\bar{\varepsilon}} v\left(a_{\sigma(1)}, \ldots, a_{\sigma(p)}\right) w\left(a_{\sigma(p+1)}, \ldots, a_{\sigma(p+q)}\right) .
$$

Here $\mathfrak{S}_{p, q} \subset \mathfrak{S}_{p+q}$ denotes the $(p, q)$-shuffles (Definition 1.2.15), and $\varepsilon$ denotes the sign of the braiding isomorphisms for the permutation $\sigma$ of $a_{i}$ 's. The $\operatorname{sign} \bar{\varepsilon}$ is given by

$$
\bar{\varepsilon}:=|w|(n+1) p+\sum_{i=1}^{k}\left|a_{\sigma(i)}\right|((n+1) q+|w|) .
$$

(2) For $v \in \operatorname{Pol}(A, n)(p)$ and $w \in \operatorname{Pol}(A, n)(q)$, the following formula gives $[v, w]_{S} \in \operatorname{Pol}(A, n)(p+q-1)$.

$$
\begin{aligned}
{[v, w]_{S}\left(a_{1}, \ldots, a_{p+q-1}\right): } & =\sum_{\sigma \in \mathfrak{S}_{q, p-1}} \operatorname{sgn}(\sigma)^{n+1}(-1)^{\varepsilon+\varepsilon_{1}} v\left(w\left(a_{\sigma(1)}, \ldots, a_{\sigma(q)}\right), a_{\sigma(q+1)}, \ldots, a_{\sigma(p+q-1)}\right) \\
& -\sum_{\sigma \in \mathfrak{S}_{p, q-1}} \operatorname{sgn}(\sigma)^{n+1}(-1)^{\varepsilon+\varepsilon_{2}} w\left(v\left(a_{\sigma(1)}, \ldots, a_{\sigma(p)}\right), a_{\sigma(p+1)}, \ldots, a_{\sigma(p+q-1)}\right) .
\end{aligned}
$$

Here $\mathfrak{S}_{p, q}$ and $(-1)^{\varepsilon}$ are the same as (1), and the $\operatorname{sign} \varepsilon_{i}$ are given by

$$
\begin{aligned}
& \varepsilon_{1}:=(n+1)(|w|+q)(p+1)+(n+1)|v|, \\
& \varepsilon_{2}:=(|v|-(n+1) p)(|w|-(n+1) q)+(n+1)(p+1)(|w|+1)+(n+1)|v| .
\end{aligned}
$$

We call the operation $[-,-]_{S}$ the Schouten bracket. It is of cohomological degree $-n-1$.

(3) The multiplication and the Schouten bracket define a dg $(n+2)$-Poisson algebra structure on $\operatorname{Pol}(A, n)$.

Here is a dg version of the classical fact [LPV, Proposition 3.5] on the Poisson structure and the Schouten bracket. We omit the proof since it is essentially the same with the classical case. 
Lemma 1.3.9. Let $n \in \mathbb{Z}, A$ be a cdga, and $\pi \in \operatorname{Pol}(A, n-1)(2)^{n+1}$ be a polyvector field of weight 2 (i.e., a bivector field) whose cohomological degree is $n+1$. We define the bilinear map

$$
\{-,-\}_{\pi}: A \otimes A \longrightarrow A[1-n], \quad\{a, b\}_{A}:=\pi(a, b),
$$

where we used Notation 1.3.7. Then $\{-,-\}_{\pi}$ defines a $\mathbb{P}_{n}$-algebra structure on $A$ if and only if $[\pi, \pi]_{S}=0$.

1.3.3. Kirillov-Kostant Poisson structure. In this part we recall the Kirillov-Kostant Poisson structure on a Lie algebra, which gives an example of a Poisson algebra arising from Lie theory.

Let $\mathfrak{l}$ be a dg Lie algebra over a field $\mathbb{k}$ with characteristics 0 . We denote the Lie bracket by $[-,-]_{\mathfrak{r}}$. Then the symmetric algebra $\operatorname{Sym}(\mathfrak{l})=\operatorname{Sym}_{\mathfrak{l}}(\mathfrak{l})$ of the underlying complex of $\mathfrak{l}$ has a structure of dg Poisson algebra with the Poisson bracket $\{-,-\}_{\text {I }}$ determined by

$$
\{x, y\}_{\mathfrak{l}}:=[x, y]_{\mathfrak{l}} \quad \text { for } x, y \in \mathfrak{l} \subset \operatorname{Sym}(\mathfrak{l})
$$

and by the Leibniz rule.

Notation 1.3.10. For a dg Lie algebra $\mathfrak{l}$, the Poisson bracket $\{-,-\}_{\mathfrak{l}}$ is called the Kirillov-Kostant Poisson bracket, and the resulting dg Poisson algebra $\left(\operatorname{Sym}(\mathfrak{l}),\{-,-\}_{\mathfrak{l}}\right)$ is called the Lie-Poisson algebra of $\mathfrak{l}$.

Recall that for a cdga $R$, we denote by $\mathbb{T}_{R}=\operatorname{Der}(R)$ the dg Lie algebra of derivations on $R$ (Example 1.2.11). If moreover $R$ is a dg Poisson algebra with the Poisson bracket $\{-,-\}_{R}$, then we have a linear map

$$
D: R \longrightarrow \operatorname{Der}(R), \quad D(r):=\{r,-\}_{R} .
$$

Definition 1.3.11. Let $R$ be a dg Poisson algebra. A Hamiltonian $\mathfrak{l}$-action on $R$ is a morphism $a: \mathfrak{l} \rightarrow$ $\operatorname{Der}(R)$ of dg Lie algebras equipped with a dg Lie algebra morphism $\mu: \mathfrak{l} \rightarrow R$ such that $D \circ \mu=a$. In this case, the morphism $\mu$ is called the momentum map.

Remark 1.3.12. (1) More explicitly, the condition is

$$
\{\mu(x), r\}_{R}=a(x)(r)
$$

for any $x \in \mathfrak{l}$ and $r \in R$. We call it the momentum map equation.

(2) An example is $R=\mathbb{k}$ with the trivial Poisson bracket. The morphism $a$ and the momentum map $\mu$ are both trivial.

(3) We can replace the momentum map $\mu: \mathfrak{l} \rightarrow R$ by the induced map Sym $\mathfrak{l} \rightarrow R$ of dg Poisson algebras. We denote it by the same symbol $\mu$ and also call it the momentum map.

1.4. Shifted Poisson structures for derived stacks. In this subsection we give a brief recollection on the theory of shifted Poisson structures on derived stacks $[\mathrm{CPT}+]$.

1.4.1. Affine derived Poisson schemes. Let us restate the notions on shifted Poisson algebras in terms of the language of affine derived schemes in the sense of [TVe, T14]. The language of derived algebraic geometry will be introduced in the later $\$ 1.4 .2$, and the present part is a preparation for it.

Hereafter we use the language of $\infty$-categories. See $\S 0.3$ for our terminology on $\infty$-categories. In particular, we will identify a category with its nerve and a dg category with its dg nerve, so that we regard them as $\infty$-categories.

Notation 1.4.1. Here is a brief list of the notations on affine derived schemes.

(1) In this part we assume that $\mathbb{k}$ contains $\mathbb{Q}$. Although we can remove this assumption, we put it to make the text simple and compatible with the literatures of derived algebraic geometry.

(2) We denote by dguCom the dg category of unital cdgas over $\mathbb{k}$, and denote by $\operatorname{dguCom}^{\leq 0} \subset$ dguCom the full sub-dg-category spanned by objects concentrated in non-positive degrees. As in $\S 0.3(5)(\mathrm{x})$, we regard these dg categories as $\infty$-categories.

(3) The $\infty$-categories dguCom and $\mathbf{d g u C o m}{ }^{\leq 0}$ are symmetric monoidal in the sense of [Lu2] with the derived tensor product $\otimes^{\mathbb{L}}$. We also have a relative version: Given two morphisms $B \rightarrow A$ and $C \rightarrow A$ of cdgas, we have the derived tensor product $B \otimes_{A}^{\mathbb{L}} C$. It is realized by the two-sided bar complex. See Definition 2.1.8 for the detail.

(4) The $\infty$-category dAff of affine derived schemes over $\mathbb{k}$ is defined to be the opposite $\infty$-category of $\operatorname{dguCom}^{\leq 0}$. For $R \in \mathbf{d g u C o m}^{\leq 0}$, we denote the corresponding affine derived scheme by $\operatorname{Spec}(R)$. Conversely, for an affine derived scheme $X=\operatorname{Spec}(R)$, we call $R$ the coordinate (derived) ring of $X$.

(5) The derived tensor product $B \otimes_{A}^{\mathbb{L}} C$ in (3) is transfered to the derived fiber product or derived intersection $\operatorname{Spec}(B) \times{ }_{\operatorname{Spec}(A)}^{\mathbb{L}} \operatorname{Spec}(C)$ in the $\infty$-category dAff of affine derived schemes. 
(6) For an affine derived scheme $X=\operatorname{Spec}(R)$, we denote the structure sheaf by $\mathcal{O}_{X}$. It is a sheaf of unital cdgas on the étale $\infty$-topos on $X$. See [TVe, Chap. 2.2] for the detail.

(7) For an affine derived scheme $X=\operatorname{Spec}(R)$, we denote by $\mathrm{L}_{\mathrm{QCoh}}(X)$ the $\infty$-category of quasi-coherent sheaves of $\mathcal{O}_{X}$-modules over $X$.

The equivalence of the (ordinary) category of quasi-coherent sheaves over an affine scheme and the category of modules over the corresponding commutative ring is enhanced to

Fact 1.4.2. For an affine derived scheme $X=\operatorname{Spec}(R)$, the $\infty$-category $\mathrm{L}_{\mathrm{QCoh}}(X)$ is equivalent to the $\infty$-category $R$-dgMod associated to the dg category of $\mathrm{dg} R$-modules.

Moreover, $\mathrm{L}_{Q}$ Coh $(X)$ is a stable $\infty$-category in the sense of [Lu2, Chap. 1]. If $X$ is an affine (non-derived) scheme, then the homotopy category of $\mathrm{L}_{\mathrm{QCoh}}(X)$ is equivalent to the derived category $\mathrm{D}_{\mathrm{Q} C o h}(X)$ of quasicoherent étale sheaves of $\mathcal{O}_{X}$-modules on $X$.

Now the following definition should be a natural one.

Definition 1.4.3. (1) The $\infty$-category of affine derived $n$-Poisson schemes is defined to be the opposite of the $\infty$-category $\mathbb{P}_{n}$-dguCom ${ }^{\leq 0}$ associated to the dg category of $\mathbb{P}_{n}$-algebras concentrated in nonpositive cohomological degrees.

(2) An object of the above $\infty$-category is called an affine derived $n$-Poisson scheme. Following the notation of affine derived schemes, we denote by $X=\operatorname{Spec}(R)$ the object associated to $R \in$ $\mathbb{P}_{n}$-dguCom ${ }^{\leq 0}$.

(3) In the case $n=1$ of (2), the object is called an affine derived Poisson scheme.

Example. Recall the Kirillov-Kostant Poisson structure on a dg Lie algebra $\mathfrak{l}$ (Notation 1.3.10). If $\mathfrak{l}$ is concentrated in non-positive cohomological degrees, then we may regard $\operatorname{Sym}(\mathfrak{l})$ as the coordinate ring of the affine derived scheme $\mathfrak{l}^{*}$. Thus we have the affine derived 1-Poisson scheme $\mathfrak{l}^{*}$.

Let $X=\operatorname{Spec}(R)$ be an affine derived $n$-Poisson scheme. Then the structure sheaf $\mathcal{O}_{X}$ is an étale sheaf both of cdgas and of $n$-shifted dg Lie algebras whose local sections satisfy the Leibniz rule.

We denote by $\mathrm{L}_{\mathrm{Pois} Q \mathrm{Coh}}(X)$ the $\infty$-category of étale sheaves on $X$ which are both quasi-coherent sheaves of $\operatorname{dg} \mathcal{O}_{X}$-modules and sheaves of $n$-shifted dg Lie superalgebra $\mathcal{O}_{X}$-modules whose local sections satisfy the relation Definition 1.3.3 (iii). Then Fact 1.4.2 naturally yields

Lemma. Let $X=\operatorname{Spec}(R)$ be an affine derived $n$-Poisson scheme. Then there is an equivalence of $\infty$ categories

$$
\operatorname{L}_{\text {PoisQCoh }}(X) \simeq R \text {-dgPMod. }
$$

1.4.2. Shifted Poisson structures and shifted symplectic structures. At this stage we recall the shifted Poisson structure for derived stacks introduced in [CPT,+ 3.1$]$. We use the language of derived stacks developed in [TVe] and its $\infty$-categorical presentation in [T14]. See Notation 1.4.1 for our notation on the affine derived schemes, and also $§ 0.3$ for our terminology on $\infty$-categories.

Notation 1.4.4. (1) We work over a field $\mathbb{k}$ which contains $\mathbb{Q}$.

(2) A geometric derived stack means an $n$-geometric derived $D^{-}$-stack over $\mathbb{k}$ with some $n \in \mathbb{Z}_{>-1}$ in the sense of [TVe, §1.3.3, §2.2.3]. In particular, the affine derived schemes (Notation 1.4.1 (4)) are $(-1)$-geometric derived stack.

(3) Algebraic stacks, algebraic spaces and schemes over $\mathbb{k}$ are regarded as geometric derived stacks as in the way of [TVe, $\S 2.1 .2, \S 2.2 .4]$.

(4) For a geometric derived stack $X$, we denote by $\mathrm{L}_{Q} \operatorname{Coh}(X)$ the derived $\infty$-category of quasi-coherent sheaves over $X$. For an affine derived scheme $X=\operatorname{Spec}(R)$, it coincides with the one in Notation 1.4.1 (7).

(5) For a geometric derived stack $X$, the cotangent complex [TVe, Chap. 1.4] is denoted by $\mathbb{L}_{X} \in$ $\mathrm{L}_{Q \operatorname{Coh}}(X)$. If $X$ is locally of finite presentation [TVe, 1.3.6], then $\mathbb{L}_{X}$ is dualizable [TVe, §1.4.1], and we denote its dual by $\mathbb{T}_{X}:=\mathbb{L}_{X}^{\vee} \in \mathrm{L}_{\mathrm{QCoh}}(X)$.

Let $X$ be a geometric derived stack locally of finite presentation. By [CPT,+ 3.1$]$, we have a graded $\mathbb{P}_{n+1}$-algebra

$$
\operatorname{Pol}(X, n)=\mathbb{R} \Gamma\left(X, \operatorname{Sym}_{\mathcal{O}_{X}}\left(\mathbb{T}_{X}[-n-1]\right)\right)
$$

whose element is called a $n$-shifted polyvector of $X$. For an affine derived scheme $X=\operatorname{Spec}(R)$, the cdga $\operatorname{Pol}(X, n)$ is quasi-isomorphic to $\operatorname{Sym}_{R}\left(\mathbb{T}_{R}[-n-1]\right)$, where $\mathbb{T}_{R}$ is the dg Lie algebra of derivations on the cdga $R$ (Example 1.2.11). 
Definition 1.4.5 ([CPT+, Definition 3.1.1]). The space of $n$-shifted Poisson structures on $X$ is the object $\operatorname{Pois}(X, n) \in \mathcal{S}$ given by

$$
\operatorname{Pois}(X, n):=\underline{\operatorname{Map}}_{\operatorname{dgLie}^{g r}}(\mathbb{k}(2)[-1], \operatorname{Pol}(X, n)[n+1]) .
$$

Here $\mathbb{k}(2)$ indicates the shift of the weight grading (Definition 1.3.6), and $\mathbf{d g L i e}{ }^{\text {gr }}$ denotes (the $\infty$-category associated to) the dg category of dg Lie algebras with extra weight gradings.

Finally we recall the relation between shifted symplectic and Poisson structures.

Definition. Let $X$ be a geometric derived stack locally of finite presentation. An $n$-shifted Poisson structure $\pi$ on $X$ is non-degenerate if the morphism $\pi^{\sharp}: \mathbb{L}_{X} \rightarrow \mathbb{T}_{X}[-n]$ induced by the underlying bivector field is an equivalence.

By [CPT + , Theorem 3.2.4], there is an equivalence between the $\infty$-category of non-degenerate $n$-shifted Poisson structures and that of shifted symplectic structures in the sense of [PTVV] on a geometric derived stack locally of finite presentation. We will not give the detail, and refer [CPT +$]$ for the detail.

In this text we only need affine versions of these notions. Let us collect them in:

Definition 1.4.6. Let $R$ be a cdga.

(1) A $\mathbb{P}_{n}$-algebra structure on $R$ is non-degenerate if the morphism $\pi^{\sharp}: \mathbb{L}_{R} \rightarrow \mathbb{T}_{R}[-n]$ induced by the underlying bivector field is a quasi-isomorphism of cdgas.

(2) A $\widehat{\mathbb{P}}_{n}$-algebra structure on $R$ is non-degenerate if the induced $\mathbb{P}_{n}$-algebra structure on the cohomology $H(R)$ (Remark 2.1.6 (2)) is non-degenerate in the sense of (1).

(3) An $(n-1)$-shifted symplectic structure on $R$ is defined to be a non-degenerate $n$-shifted Poisson structure on $R$.

\section{Derived Hamiltonian Reduction and Classical BRST complex}

In this section we review the work of Safronov [S17] on the reduction of shifted Poisson algebras and its relation to the classical BRST complex. We work over a field $\mathbb{k}$ with characteristics 0 .

2.1. Derived Hamiltonian reduction. In this subsection we cite from [S17, §1] the reduction of shifted Poisson structure in terms of derived coisotropic intersection.

2.1.1. Coisotropic structure. In this part we explain the coisotropic structure for shifted Poisson algebras. It is an enhancement of the notion of coisotropic subschemes of affine Poisson schemes. and also gives the notion of modules over shifted Poisson algebras. In the next $\$ 2.1 .2$, we explain the construction of homotopy shifted Poisson algebras via coisotropic structures.

Let $R$ be a $\mathbb{P}_{n}$-algebra. Then by Lemma 1.3.9, we have a bivector field $\pi_{R} \in \operatorname{Pol}(R, n-1)(2)$ with the Schouten bracket $\left[\pi_{R}, \pi_{R}\right]_{S}=0$ which corresponds to the Poisson bracket of $R$. On the other hand, we have the $\operatorname{dg} R$-module $\Omega_{R}^{1}$ of Kähler differentials (Notation 1.3.4). We denote its differential by $d_{\Omega_{R}^{1}}$.

Definition 2.1.1. For a $\mathbb{P}_{n}$-algebra $R$, its Poisson center is the $\mathbb{P}_{n+1}$-algebra

$$
Z(R)=\left(\widehat{\operatorname{Pol}}(R, n-1), d_{Z(R)},\{\cdot, \cdot\}_{Z(R)}\right)
$$

in $d g \vee e c$ where

- the underlying linear space is defined to be the completion

$$
\widehat{\operatorname{Pol}}(R, n-1):=\underline{\operatorname{Hom}}_{R}\left(\widehat{\operatorname{Sym}}_{R}\left(\Omega_{R}^{1}[n]\right), R\right)
$$

of $\operatorname{Pol}(R, n-1)=\underline{\operatorname{Hom}}_{R}\left(\operatorname{Sym}_{R}\left(\Omega_{R}^{1}[n]\right), R\right)$ with respect to the weight grading, and

- the $\mathrm{dg}(n+1)$-Poisson structure is induced by that on $\operatorname{Pol}(R, n-1)$ (Fact 1.3.8). In particular, the Lie bracket is the Schouten bracket.

We also denote by

$$
p_{R}: Z(R) \longrightarrow R
$$

the morphism in dguCom given by the projection to the weight 0 part of polyvector fields.

We give an example of Poisson center which can be described explicitly. Let $\mathfrak{l}$ be a dg Lie algebra. Recall the Kirillov-Kostant Poisson structure on $\operatorname{Sym}(\mathfrak{l})$ (Definition 1.3.10), which makes $\operatorname{Sym}(\mathfrak{l})$ a dg Poisson algebra. Thus we can consider its Poisson center.

Lemma 2.1.2 ([S17, §2.1]). The Poisson center $Z(\operatorname{Sym}(\mathfrak{l}))$ of the Lie-Poisson algebra $\operatorname{Sym}(\mathfrak{l})$ is the dg 2-Poisson algebra of which 
- the underlying cdga is the completion $\widehat{\mathrm{CE}}(\mathfrak{l}, R)$ of the Chevalley-Eilenberg cdga $\mathrm{CE}(\mathfrak{l}, R)$ (Lemma 1.2.17) with respect to the weight grading, and

- the 2-Poisson bracket is given by the Schouten bracket (Fact 1.3.8).

Proof. Denoting $R:=\operatorname{Sym}(\mathfrak{l})$, the module $\Omega_{R}^{1}$ of Kähler differentials is a free $\operatorname{dg} R$-module generated by $\mathfrak{l}$ (Example 1.3.5). Thus we have an isomorphism

$$
Z(R)=\widehat{\operatorname{Pol}}(R, 0)=\prod_{p \in \mathbb{N}} \underline{\operatorname{Hom}}_{R}\left(\operatorname{Sym}_{R}(\mathfrak{l}[1])(p), R\right) \simeq \prod_{p \in \mathbb{N}} \underline{\operatorname{Hom}}(\operatorname{Sym}(\mathfrak{l}[1])(p), R) \simeq \widehat{\operatorname{CE}}(\mathfrak{l}, R)
$$

of graded linear spaces. The compatibility of the dg 2-Poisson structure can be checked by comparing that on $\mathrm{CE}(\mathfrak{l}, R)$ (Remark 1.2.14, Definition 1.2.16) and that on $Z(R)$ (Fact 1.3.8).

Now we given the definition of coisotropic structure.

Definition 2.1.3. Let $R$ be a $\mathbb{P}_{n+1}$-algebra and $M$ be a $\mathbb{P}_{n}$-algebra. A coisotropic morphism is a morphism $f: R \rightarrow M$ in dguCom equipped with a morphism $\tilde{f}: R \rightarrow Z(M)$ of $\mathbb{P}_{n+1}$-algebras such that $f=p_{M} \circ \tilde{f}$ holds in dguCom.

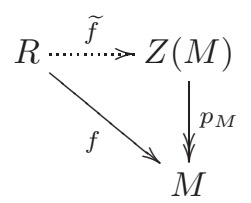

Remark 2.1.4. (1) We can rewrite the definition of a coisotropic morphism as a family

$$
\left\{f_{p}: A \longrightarrow \underline{\operatorname{Hom}}_{M}\left(\operatorname{Sym}\left(\Omega_{M}^{1}[n]\right)(p), M\right) \mid p \in \mathbb{N}\right\}
$$

with $f_{0}=f: A \rightarrow M$ which satisfies some compatibility conditions. See [S17, 11.3$]$ for the detail.

(2) Let us explain the origin of the name "coisotropic morphism". The compatibility conditions imply that the kernel of $f_{0}$ is closed under the Poisson bracket. Thus, if $A$ and $M$ are (non-dg) commutative algebras, then $\operatorname{Spec}(M) \rightarrow \operatorname{Spec}(A)$ is a coisotropic subscheme in the ordinary sense. See [S17, Remark 1.9] for the detail.

2.1.2. Coisotropic intersection. We turn to a construction of homotopy shifted Poisson algebras by the "derived" intersection of coisotropic structures, It will be used for the Poisson reduction in the next §2.1.3.

We start with the definition of homotopy shifted Poisson algebra. Abstractly, we replace the Lie algebra structure by homotopy Lie algebra structure, i.e., $L_{\infty}$-algebra structure. Explicitly, we have:

Definition 2.1.5 ([S17, §1.2]). (1) A $\widehat{\mathbb{P}}_{n}$-algebra in $\mathrm{dgVec}$, or a homotopy $n$-Poisson algebra is a cdga $A=\left(A, d_{A}, \cdot\right)$ equipped with a family

$$
\left\{l_{p}: A^{\otimes p} \rightarrow A[(p-1) n-1] \mid p \in \mathbb{N}\right\}
$$

of morphisms in dguCom satisfying the following relations.

(i) $l_{1}=d_{A}$,

(ii) $l_{p}\left(a_{1}, \ldots, a_{p}\right)=(-1)^{\left|a_{i}\right|\left|a_{i+1}\right|+n} l_{p}\left(a_{1}, \ldots, a_{i+1}, a_{i}, \ldots, a_{p}\right)$.

(iii) $l_{p}\left(a_{1}, \ldots, a_{p} \cdot a_{p+1}\right)=l_{p}\left(a_{1}, \ldots, a_{p}\right) \cdot a_{p+1}+(-1)^{\left|a_{p}\right|\left|a_{p+1}\right|} l_{p}\left(a_{1}, \ldots, a_{p-1}, a_{p+1}\right) \cdot a_{p}$.

(iv) $\sum_{q=1}^{p}(-1)^{n q(p-q)} \sum_{\sigma \in \mathfrak{S}_{q, p-q}} \operatorname{sgn}(\sigma)^{n}(-1)^{\varepsilon} l_{p-q+1}\left(l_{q}\left(a_{\sigma(1)}, \ldots, a_{\sigma(q)}\right), a_{\sigma(q+1)}, \ldots, a_{\sigma(p)}\right)=0$, where $\mathfrak{S}_{q, p-q}$ and $\varepsilon$ are the same as Definition 1.2.16.

(2) For a $\widehat{\mathbb{P}}_{n}$-algebra $A$, we define the opposite algebra $A^{\text {op }}$ to be the $\widehat{\mathbb{P}}_{n}$-algebra with the same underlying cdga and $l_{p}^{\mathrm{op}}:=(-1)^{p+1} l_{p}$.

Remark 2.1.6. (1) A $\mathbb{P}_{n}$-algebra is nothing but a $\widehat{\mathbb{P}}_{n}$-algebra with $l_{p}=0$ for $p \geq 3$ and $l_{2}=\{-,-\}$.

(2) For a $\widehat{\mathbb{P}}_{n}$-algebra $A$, the underlying cohomology $H\left(A, d_{A}\right)$ carries a natural $\mathbb{P}_{n}$-algebra structure.

(3) As mentioned in Remark 1.3.2, there is a dg operad $\mathbb{P}_{n}$ such that a $\mathrm{dg} n$-Poisson algebra is an algebra over $\mathbb{P}_{n}$ in dgVec. Similarly, we can construct an operad $\widehat{\mathbb{P}}_{n}$ such that a homotopy $n$-Poisson algebra is nothing but an algebra over $\widehat{\mathbb{P}}_{n}$.

Finally we can explain:

Fact 2.1.7 ([S17, Theorem 1.18]). Let $R$ be a $\mathbb{P}_{n+1}$-algebra, $M$ and $N$ be $\mathbb{P}_{n}$-algebras, and $f: R \rightarrow M$ and $g: R \rightarrow N$ be coisotropic morphisms. Then the two sided bar complex

$$
M \otimes_{f, R, g}^{\mathbb{L}} N=M \otimes_{R}^{\mathbb{L}} N
$$

has a structure of a $\widehat{\mathbb{P}}_{n}$-algebra such that the projection $M^{\mathrm{op}} \otimes N \rightarrow M \otimes \frac{\mathbb{L}}{R}$ is a morphism of $\widehat{\mathbb{P}}_{n}$-algebras. 
Let us explain the detail. Recall first the two-sided bar complex:

Definition 2.1.8. Let $A$ be a dg algebra, $L$ be a left $\operatorname{dg} A$-module, and $M$ be a right $\operatorname{dg} A$-module $M$. We consider the double complex

$$
B^{-p, q}:=\bigoplus_{i+j+k=q} L^{i} \otimes\left(A[1]^{\otimes p}\right)^{j} \otimes M^{k} \quad(p \in \mathbb{N}, q \in \mathbb{Z})
$$

with the differentials

$$
d_{v}^{-p, q}: B^{-p, q} \longrightarrow B^{-p, q+1}, \quad d_{h}^{-p, q}: B^{-p, q} \longrightarrow B^{-p+1, q} .
$$

The vertical differential $d_{v}$ is the one on the tensor product $B \otimes T(A[1]) \otimes C$ of complexes (Definition 1.1.4). The horizontal differential $d_{h}$ is given by

$$
d_{h}^{-p, q}\left[l\left|a_{1}\right| \cdots\left|a_{p}\right| m\right]=\left[l . a_{1}|\cdots| a_{p} \mid m\right]+\sum_{i=1}^{p-1}(-1)^{i}\left[l|\cdots| a_{i} a_{i+1}|\cdots| m\right]+(-1)^{p}\left[l|\cdots| a_{p} \cdot m\right] .
$$

Here we denoted by

$$
\left[l\left|a_{1}\right| \cdots\left|a_{p}\right| m\right] \in L \otimes A^{\otimes p} \otimes M
$$

with $l \in L, a_{i} \in A$ and $m \in M$. Note that we have $(A[1])^{\otimes p}=A^{\otimes p}[p]$. Now the complex $L \otimes \mathbb{L}_{A}^{\mathbb{L}} M$ is defined to be the total complex

$$
L \otimes_{A}^{\mathbb{L}} M:=\left(\operatorname{Tot}\left(B^{\bullet}, \bullet\right),(-1)^{p} d_{v}^{-p, q}+d_{h}^{-p, q}\right)
$$

Next we cite some constructions from [S17]. The fist one is:

Fact 2.1.9 ([S17, Proposition 1.14]). Let $A$ be a $\mathbb{P}_{n+1}$-algebra. Then the graded linear space

$$
T(A[1])=\bigoplus_{p \in \mathbb{N}} A^{\otimes p}[p]
$$

has a $\mathbb{P}_{n}$-bialgebra structure $\left(d_{\mathrm{bar}}, \cdot, \Delta_{\mathrm{dec}},\{-,-\}\right)$.

In other words, $\left(T(A[1]), d_{\mathrm{bar}}, \cdot,\{-,-\}\right)$ is a $\mathbb{P}_{n}$-algebra and

$$
\Delta_{\mathrm{dec}}: T(A[1]) \otimes T(A[1]) \longrightarrow T(A[1])
$$

is a coassociative comultiplication which is a morphism of $\mathbb{P}_{n}$-algebras.

In order to write down the $\mathbb{P}_{n}$-bialgebra structure, let us denote an element of $A^{\otimes p}$ by $\left[a_{1}|\cdots| a_{p}\right]$ with $a_{i} \in A$, following the notation in [S17]. Then the structure is given by

- The differential $d_{\text {bar }}$ is the bar differential:

$$
\begin{aligned}
d_{\mathrm{bar}}\left[a_{1}|\cdots| a_{p}\right] & =\sum_{i=1}^{p}(-1)^{\sum_{j=1}^{i-1}\left|a_{j}\right|+i-1}\left[a_{1}|\cdots| d_{A} a_{i}|\cdots| a_{p}\right] \\
& +\sum_{i=1}^{p-1}(-1)^{\sum_{j=1}^{i}\left|a_{j}\right|+i}\left[a_{1}|\cdots| a_{i} a_{i+1}|\cdots| a_{p}\right] .
\end{aligned}
$$

Note that as a map on $T(A[1])$ it is indeed of cohomological degree 1 .

- The multiplication - is the shuffle product:

$$
\left[a_{1}|\cdots| a_{p}\right] \cdot\left[a_{p+1}|\cdots| a_{p+q}\right]=\sum_{\sigma \in \mathfrak{S}_{p, q}}(-1)^{\varepsilon}\left[a_{\sigma(1)}|\cdots| a_{\sigma(p+q)}\right],
$$

where $\mathfrak{S}_{p, q}$ is the set of shuffles and $\varepsilon$ is the same braiding sign as in Definition 1.2.16.

- The comultiplication $\Delta_{\text {dec }}$ is the deconcatenation coproduct:

$$
\Delta_{\text {dec }}\left[a_{1}|\cdots| a_{p}\right]=\sum_{i=0}^{p}\left[a_{1}|\cdots| a_{i}\right] \otimes\left[a_{i+1}|\cdots| a_{p}\right] .
$$

- The Lie bracket is

$$
\begin{gathered}
\left\{\left[a_{1}|\cdots| a_{p}\right],\left[b_{1}|\cdots| b_{q}\right]\right\}=\sum_{i=1}^{p} \sum_{j=1}^{q}(-1)^{\varepsilon_{1}+\left|a_{i}\right|+n+1}\left(\left[a_{1}|\cdots| a_{i-1}\right] \cdot\left[b_{1}|\cdots| b_{j-1}\right]\right) \wedge\left[\left\{a_{i}, b_{j}\right\}\right] \\
\wedge\left(\left[a_{i+1}|\cdots| a_{p}\right] \cdot\left[b_{j+1}|\cdots| b_{q}\right]\right)
\end{gathered}
$$

where $\wedge$ denotes the concatenation: $\left[a_{1}|\cdots| a_{i}\right] \wedge\left[a_{i+1}|\cdots| a_{p}\right]=\left[a_{1}|\cdots| a_{p}\right]$.

Remark. The bialgebra structure coincides with the bialgebra $\left(T(V), \mu^{\prime}, \Delta\right)$ in $[\mathrm{LV}, \S 1.3 .2]$, which is the one of the two standard bialgebra structures on the tensor space $T(V)$.

The second construction is:

Fact 2.1.10 ([S17, Proposition 1.17]). Let $A$ be a $\mathbb{P}_{n+1}$-algebra, $M$ be a $\mathbb{P}_{n}$-algebra and $f: A \rightarrow M$ be a coisotropic morphism. Then the tensor product $T(A[1]) \otimes M$ of graded linear spaces has a left $\widehat{\mathbb{P}}_{n}$-comodule structure $\left(d_{\text {bar }}, \cdot,\left\{l_{k} \mid k \in \mathbb{N}\right\}, c\right)$ over the $\mathbb{P}_{n}$-bialgebra $T(A[1])$ in Fact 2.1.9. 
In other words, $\left(T(A[1]) \otimes M, d_{\text {bar }}, \cdot,\left\{l_{k} \mid k \in \mathbb{N}\right\}\right)$ is a $\widehat{\mathbb{P}}_{n}$-bialgebra and

$$
c: T(A[1]) \otimes M \longrightarrow T(A[1]) \otimes(T(A[1]) \otimes M)
$$

is a coassociative left coaction map which is also a morphism of $\widehat{\mathbb{P}}_{n}$-algebras.

As in the explanation of the $\mathbb{P}_{n}$-bialgebra $T(A[1])$, we denote by $\left[a_{1}|\cdots| a_{p} \mid m\right]$ an element of $A^{\otimes p} \otimes M$. Then the left $\widehat{\mathbb{P}}_{n}$-comodule structure is given as follows.

- The differential $d_{\text {bar }}$ is the bar differential:

$$
\begin{aligned}
d_{\mathrm{bar}}\left[a_{1}|\cdots| a_{p} \mid m\right] & =\sum_{i=1}^{p}(-1)^{\sum_{j=1}^{i-1}\left|a_{j}\right|+i-1}\left[a_{1}|\cdots| d_{A} a_{i}|\cdots| a_{p} \mid m\right]+(-1)^{\sum_{j=1}^{p}\left|a_{j}\right|+p}\left[a_{1}|\cdots| a_{p} \mid d_{M} m\right] \\
& +\sum_{i=1}^{p-1}(-1)^{\sum_{j=1}^{i}\left|a_{j}\right|+i}\left[a_{1}|\cdots| a_{i} a_{i+1}|\cdots| a_{p}\right]+(-1)^{\sum_{j=1}^{p}\left|a_{j}\right|+p}\left[a_{1}|\cdots| f\left(a_{p}\right) m\right] .
\end{aligned}
$$

- The multiplication on the component $T(A[1])$ is the shuffle product (2.1), and the one on the component $M$ is that of the given cdga structure of $M$.

- As for the $L_{\infty}$-operations $l_{p}$ 's, it is enough to give them for the arguments in $T(A[1])$ or in $M$ since we have $\left[a_{1}|\cdots| a_{p} \mid m\right]=\left[a_{1}|\cdots| a_{p} \mid 1_{M}\right] \cdot[m]$, They are given by

$$
\begin{aligned}
& l_{p+1}\left(\left[a_{1}|\cdots| a_{q} \mid 1_{M}\right],\left[m_{1}\right], \cdots,\left[m_{p}\right]\right)=(-1)^{\left(\sum_{i=1}^{q}\left|a_{i}\right|+q\right)(1-n p)}\left[a_{1}|\cdots| a_{q-1} \mid f_{p}\left(a_{q}\right)\left(m_{1}, \ldots, m_{p}\right)\right], \\
& l_{2}\left(\left[m_{1}\right],\left[m_{2}\right]\right)=\left[\left\{m_{1}, m_{2}\right\}_{M}\right],
\end{aligned}
$$

and all the other operations are defined to be zero. Here $f_{p}: A \rightarrow \underline{\operatorname{Hom}}_{M}\left(\operatorname{Sym}\left(\Omega_{M}^{1}[n]\right)(p), M\right)$ is the map associated to the coisotropic morphism explained in Remark 2.1.4, and we use the notation (1.2) to get $f_{p}\left(a_{q}\right)\left(m_{1}, \ldots, m_{p}\right) \in M$.

- The coaction map $c$ is the one induced by the multiplication on $T(A[1])$.

Finally we can explain the outline of the proof of Fact 2.1.7. Let $A$ be a $\mathbb{P}_{n+1^{-}}$-algebra, $L, M$ be a $\mathbb{P}_{n^{-}}$ algebra, and $A \rightarrow L, A \rightarrow N$ be coisotropic morphisms. We denote by $\widetilde{A}:=T(A[1])$ the $\mathbb{P}_{n}$-bialgebra (Fact 2.1.9), and by $\widetilde{N}:=\widetilde{A} \otimes N$ the left $\widehat{\mathbb{P}}_{n}$-comodule over $\widetilde{A}$ (Fact 2.1.10). In an opposite way, we can construct a right $\widehat{\mathbb{P}}_{n}$-comodule structure over $\widetilde{A}$ on $\widetilde{M}:=M \otimes \widetilde{A}$.

The tensor product $\widetilde{M} \otimes \widetilde{N}$ has a $\widehat{\mathbb{P}}_{n}$-structure induced by those on $\widetilde{M}$ and $\widetilde{N}$. Now consider the cotensor product

$$
\widetilde{M} \otimes \widetilde{A} \widetilde{N}:=\operatorname{Eq}\left(\widetilde{M} \otimes \widetilde{N} \underset{\operatorname{id} \otimes c_{N}}{\stackrel{c_{M} \otimes \mathrm{id}}{\rightrightarrows}} \widetilde{M} \otimes \widetilde{A} \otimes \widetilde{N}\right),
$$

where $c_{M}$ and $c_{N}$ denote coactions on $\widetilde{M}$ and $\widetilde{N}$ respectively, and the equalizer means the strict equalizer in the category dgVec. Then we can check that the cotensor product is a $\widehat{\mathbb{P}}_{n}$-subalgebra of $\widetilde{M} \otimes \widetilde{N}$.

It is now enough to construct an isomorphism $\widetilde{M} \otimes^{\widetilde{A}} \widetilde{N} \simeq M \otimes_{A}^{\mathbb{L}} N$ of cdgas. Note that the coassociativity of the comultiplication $\Delta: \widetilde{A} \rightarrow \widetilde{A} \otimes \widetilde{A}$ yields an isomorphism

$$
\widetilde{\Delta}: \widetilde{A} \stackrel{\sim}{\longrightarrow} \operatorname{Eq}(\widetilde{A} \otimes \widetilde{A} \underset{\mathrm{id} \otimes \Delta}{\stackrel{\Delta \otimes \mathrm{id}}{\rightrightarrows}} \widetilde{A} \otimes \widetilde{A} \otimes \widetilde{A})
$$

of cdgas. Thus we have the desired isomorphism of cdgas:

$$
M \otimes_{A}^{\mathbb{L}} N=M \otimes \widetilde{A} \otimes N \underset{\mathrm{id} \otimes \widetilde{\Delta} \otimes \mathrm{id}}{\stackrel{\sim}{\longrightarrow}} \operatorname{Eq}\left(M \otimes \widetilde{A}^{\otimes 2} \otimes N \rightrightarrows M \otimes \widetilde{A}^{\otimes 3} \otimes N\right)=\widetilde{M} \otimes^{\widetilde{A}} \tilde{N} .
$$

2.1.3. Poisson reduction with respect to momentum maps. In this part we explain the work of Safronov on the reduction of Poisson algebras following [S17, §2].

Let $R$ be a dg Poisson algebra with a Hamiltonian l-action (Definition 1.3.11), and denote by $\mu: \mathfrak{l} \rightarrow R$ the associated momentum map. Then $R$ can be regarded as a dg l-module, and the momentum map gives a morphism $\mu: \operatorname{Sym}(\mathfrak{l}) \rightarrow R$ of dg Poisson algebras (see Remark 1.3.12 (3)). Thus the functor CE(l, - ) yields a morphism

$$
\mathrm{CE}(\mathfrak{l}, \mu): \operatorname{CE}(\mathfrak{l}, \operatorname{Sym}(\mathfrak{l})) \rightarrow \mathrm{CE}(\mathfrak{l}, R)
$$

in $\mathfrak{l}$-dgMod. Since $\operatorname{Sym}(\mathfrak{l})$ and $R$ are commutative ring objects in $\mathfrak{l}$-dgMod, $\operatorname{CE}(\mathfrak{l},-)$ yields the ChevalleyEilenberg cdgas (Lemma 1.2.17), and the map $\mathrm{CE}(\mathfrak{l}, \mu)$ is also a morphism in dguCom.

If moreover $\mathfrak{l}$ is of finite dimension and concentrated in non-positive cohomological degrees, then by Remark 1.2.14 (3) we have

$$
\mathrm{CE}(\mathfrak{l}, R) \simeq \operatorname{Sym}\left(\mathfrak{l}^{*}[-1]\right) \otimes R
$$

as cdgas. Thus, the 1-Poisson structure on $R$ induces a 1-Poisson structure on $\mathrm{CE}(\mathfrak{l}, R)$. On the other hand, as for the Poisson center $Z(\operatorname{Sym}(\mathfrak{l}))$ of the dg Poisson algebra $\operatorname{Sym}(\mathfrak{l})$, we have

$$
Z(\operatorname{Sym}(\mathfrak{l})) \simeq \widehat{\operatorname{CE}}(\mathfrak{l}, \operatorname{Sym}(\mathfrak{l}))=\underline{\operatorname{Hom}}(\widehat{\operatorname{Sym}}(\mathfrak{l}[1]), \operatorname{Sym}(\mathfrak{l}))=\underline{\operatorname{Hom}}(\operatorname{Sym}(\mathfrak{l}[1]), \operatorname{Sym}(\mathfrak{l}))=\operatorname{CE}(\mathfrak{l}, \operatorname{Sym}(\mathfrak{l})) .
$$


Here the first isomorphism is the one in Lemma 2.1.2, and the second equality comes from $\widehat{\operatorname{Sym}}(\mathfrak{r}[1])=$ $\widehat{\bigwedge}(\mathfrak{l})=\bigwedge(\mathfrak{l})=\operatorname{Sym}(\mathfrak{l}[1])$, where we used the assumption on $\mathfrak{l}$.

The present situation is summarized as the following diagram in dguCom:

$$
Z(\operatorname{Sym}(\mathfrak{l}))=\operatorname{CE}(\mathfrak{l}, \operatorname{Sym}(\mathfrak{l}))
$$

We now cite a key fact from [S17]. Note that the $\operatorname{cdga} \operatorname{CE}(\mathfrak{l}, \operatorname{Sym}(\mathfrak{l}))$ is generated by $\operatorname{CE}(\mathfrak{l}, \mathbb{k})$ and $\mathfrak{l}$.

Fact 2.1.11 ([S17, Proposition 2.5]). Let $\mathfrak{l}$ be a dg Lie algebra which is of finite dimension and concentrated in non-positive cohomological degrees. Let also $R$ be a $\mathrm{dg}$ Poisson algebra with a Hamiltonian $\mathfrak{l}$-action, and denote by $\mu: \operatorname{Sym}(\mathfrak{l}) \rightarrow R$ the associated momentum map (of dg Poisson algebras). Then the morphism

$$
\mathrm{CE}(\mathfrak{l}, \mu): \mathrm{CE}(\mathfrak{l}, \operatorname{Sym}(\mathfrak{l})) \longrightarrow \mathrm{CE}(\mathfrak{l}, R)
$$

of cdgas is a coisotropic morphism (Definition 2.1.3) with the lifting

$$
\mathrm{CE}(\mathfrak{l}, \operatorname{Sym}(\mathfrak{l})) \longrightarrow Z(\mathrm{CE}(\mathfrak{l}, R)) \simeq \mathrm{CE}\left(\mathfrak{l}, \widehat{\operatorname{Sym}}\left(\mathbb{T}_{M}[-1]\right) \otimes \widehat{\operatorname{Sym}}(\mathfrak{l})\right)
$$

given by the natural embedding $\mathrm{CE}(\mathfrak{l}, \mathbb{k}) \hookrightarrow \mathrm{CE}\left(\mathfrak{l}, \widehat{\operatorname{Sym}}\left(\mathbb{T}_{M}[-1]\right) \otimes \widehat{\operatorname{Sym}}(\mathfrak{l})\right)$ and $x \mapsto \mu(x)-x$ for $x \in \mathfrak{l}$.

In particular, we can apply this claim to the trivial $\mathfrak{l}$-module $R=\mathbb{k}$ by Remark $1.3 .12(2)$, and find that the morphism

$$
\mathrm{CE}(\mathfrak{l}, 0): \operatorname{CE}(\mathfrak{l}, \operatorname{Sym}(\mathfrak{l})) \rightarrow \mathrm{CE}(\mathfrak{l}, \mathbb{k})
$$

is coisotropic. Given another dg Poisson algebra $R$ with momentum map $\mu$, we can then apply Fact 2.1.7 to the coisotropic morphisms $\mathrm{CE}(\mathfrak{l}, \mu)$ and $\mathrm{CE}(\mathfrak{l}, 0)$ to obtain a $\widehat{\mathbb{P}}_{1}$-algebra structure on the complex

$$
\mathrm{CE}(\mathfrak{l}, \mathbb{k}) \bigotimes_{\mathrm{CE}(\mathfrak{l}, 0), \mathrm{CE}(\mathfrak{l}, \operatorname{Sym}(\mathfrak{l})), \mathrm{CE}(\mathfrak{l}, \mu)}^{\mathbb{L}} \mathrm{CE}(\mathfrak{l}, R) .
$$

Definition 2.1.12. Let $\mathfrak{l}, R$ and $\mu$ be as in Fact 2.1.11. The $\widehat{\mathbb{P}}_{1}$-algebra obtained above is denoted by

$$
R / /{ }_{\mu}^{\mathbb{L}} \operatorname{Sym}(\mathfrak{l}):=\operatorname{CE}(\mathfrak{l}, \mathbb{k}) \otimes_{\mathrm{CE}(\mathfrak{l}, \operatorname{Sym}(\mathfrak{l}))}^{\mathbb{L}} \mathrm{CE}(\mathfrak{l}, R),
$$

and is called the derived Hamiltonian reduction of $R$ with respect to the momentum map $\mu$.

Recall now the non-degeneracy of shifted Poisson structure (Definition 1.4.6 (1)). We have a relative analogue: A coisotropic morphism $f: A \rightarrow M$ from a $\mathbb{P}_{n+1}$-algebra $A$ to a $\mathbb{P}_{n}$-algebra $M$ is called nondegenerate if the Poisson structure on $A$ is non-degenerate and the induced morphism $\mathbb{L}_{M / A} \rightarrow \mathbb{T}_{A}[-n]$ is an equivalence, where $\mathbb{L}_{M / A}$ is the relative cotangent complex.

Lemma 2.1.13. Let $\mathfrak{l}, R$ and $\mu: \operatorname{Sym}(\mathfrak{l}) \rightarrow R$ be as in Fact 2.1.11, and assume that the Poisson structure on $R$ is non-degenerate.

(1) The coisotropic morphism $\mathrm{CE}(\mathfrak{l}, \mu): \mathrm{CE}(\mathfrak{l}, \operatorname{Sym}(\mathfrak{l})) \rightarrow \mathrm{CE}(\mathfrak{l}, R)$ is non-degenerate.

(2) The Poisson structure on the derived Hamiltonian reduction $R / / \mathbb{L}_{\mu}^{\mathbb{L}} \operatorname{Sym}(\mathfrak{l})$ is non-degenerate.

Proof. (1) The non-derived case is standard, and the same proof works.

(2) By [MS18, Theorem 4.23], a coisotropic structure is equivalent to a Lagrangian structure between shifted symplectic structures. The derived intersection of Lagrangians is also a Lagrangian by [C14, Theorem 3.1]. Combining these results and (1), we have the consequence.

2.2. Classical BRST complex. In this subsection we introduce the classical BRST complex for a dg Poisson algebra, and explain the relation to the Poisson reduction revealed by Safronov [S17, §2.3]. We continue to work over a field $\mathbb{k}$ with characteristics 0 .

We begin with the recollection on the classical Clifford algebra. First we give an implicit definition following [BD, 1.4.18, Examples (iii)].

(1) Let $\mathfrak{l}$ be a dg Lie algebra, and $\mathfrak{l}^{\mathfrak{b}}=\mathfrak{l} \oplus \mathbb{k}$ be a one-dimensional central extension. We define the twisted symmetric algebra $\operatorname{Sym}^{b}(\mathfrak{l})$ to be the quotient of the symmetric algebra $\operatorname{Sym}\left(\mathfrak{l}^{\mathfrak{b}}\right)$ by the ideal generated by the difference of embeddings $\mathbb{k}=\operatorname{Sym}\left(\mathfrak{l}^{\mathfrak{b}}\right)^{0} \hookrightarrow \operatorname{Sym}\left(\mathfrak{l}^{\mathfrak{b}}\right)$ and $\mathbb{k} \hookrightarrow \mathfrak{l}^{\mathfrak{b}}=\operatorname{Sym}\left(\mathfrak{l}^{\mathfrak{b}}\right)^{1} \hookrightarrow \operatorname{Sym}\left(\mathfrak{l}^{\mathfrak{b}}\right)$. This quotient inherits the Poisson bracket from the Kirillov-Kostant bracket on $\operatorname{Sym}\left(\mathfrak{l}^{\mathfrak{b}}\right)(\S 1.3 .3)$. Thus we obtain a dg Poisson algebra $\operatorname{Sym}^{b}(\mathfrak{l})$. 
(2) Let $U$ be a complex. We denote by $U^{*}=\underline{\operatorname{Hom}}(U, \mathbb{k})$ the dual complex, and by $\langle\cdot, \cdot\rangle: U^{*} \otimes U \rightarrow \mathbb{k}$ the natural pairing. Regard $U[1] \oplus U^{*}[-1]$ as a commutative dg Lie algebra, and let $\left(U[1] \oplus U^{*}[-1]\right)^{b}$ be the one-dimensional central extension determined by the pairing $\langle\cdot, \cdot\rangle$. Now we apply the construction (1) to $\left(U[1] \oplus U^{*}[-1]\right)^{b}$, and obtain a dg Poisson algebra $\operatorname{Sym}^{b}\left(U[1] \oplus U^{*}[-1]\right)$.

Definition 2.2.1. For a complex $U$, we denote the above dg Poisson algebra by

$$
\overline{\mathrm{Cl}}(U):=\operatorname{Sym}^{\mathrm{b}}\left(U[1] \oplus U^{*}[-1]\right)
$$

and call it the classical Clifford algebra.

On the naming we refer Remark 4.3.13.

The classical Clifford algebra $\overline{\mathrm{Cl}}(U)$ is explicitly described as follows.

- As a commutative dg algebra, we have $\overline{\mathrm{Cl}}(U)=\operatorname{Sym}\left(U[1] \oplus U^{*}[-1]\right)$.

- Taking a linear basis $\left\{u_{i} \mid i \in I\right\}$ of $U$ and its dual basis of $U^{*}$, we denote the corresponding algebra generators by

$$
\bar{\psi}_{i} \in U[1], \quad \bar{\psi}_{i}^{*} \in U^{*}[-1] \quad(i \in I) .
$$

The Poisson bracket is determined by the Leibniz rule and

$$
\left\{\bar{\psi}_{i}, \bar{\psi}_{j}\right\}=0=\left\{\bar{\psi}_{i}^{*}, \bar{\psi}_{j}^{*}\right\}, \quad\left\{\bar{\psi}_{i}, \bar{\psi}_{j}^{*}\right\}=\delta_{i, j} \quad(i, j \in I) .
$$

- $\overline{\mathrm{Cl}}(U)$ has an extra $\mathbb{Z}$-grading, called the charge grading, given by

$$
\operatorname{charge}\left(\bar{\psi}_{i}\right)=-1, \quad \operatorname{charge}\left(\bar{\psi}_{i}^{*}\right)=1 .
$$

If $U$ is concentrated in cohomological degree 0 , then we recover the standard definition

$$
\overline{\mathrm{Cl}}(U) \simeq \bigwedge(U) \otimes \bigwedge\left(U^{*}\right)
$$

as the tensor product of the exterior algebras. The charge grading is given by

$$
\overline{\mathrm{Cl}}^{n}(U)=\bigoplus_{\substack{p, q \in \mathbb{N} \\-p+q=n}} \bigwedge^{p}(U) \otimes \bigwedge^{q}\left(U^{*}\right) .
$$

Next we introduce the classical BRST complex. Following [BD, 1.4.23-1.4.25], we give a construction in the next lemma. We can check the statements by direct computation, and omit the proofs.

Lemma 2.2.2. Let $\mathfrak{l}$ be a dg Lie algebra, and $R$ be a dg Poisson algebra equipped with a Hamiltonian l-action (Definition 1.3.11). The momentum map is denoted by $\mu: \mathfrak{l} \rightarrow R$.

(1) The adjoint action of $\mathfrak{l}$ on itself yields a morphism of $d g$ Lie algebras

$$
\beta: \mathfrak{l} \longrightarrow \mathfrak{l} \otimes \mathfrak{l}^{*} \stackrel{\sim}{\longrightarrow} \mathfrak{l}[1] \otimes \mathfrak{l}^{*}[-1] \hookrightarrow \overline{\mathrm{Cl}}(\mathfrak{l})^{0} .
$$

(2) Recall the contractible dg Lie algebra $\mathfrak{l}_{\dagger}(\S 1.2 .4)$. Let

$$
\ell: \mathfrak{l}_{\dagger} \longrightarrow \overline{\mathrm{Cl}}(\mathfrak{l}) \otimes R
$$

be the morphism of graded linear spaces given by

$$
\ell^{0}:=1 \otimes \mu+\beta \otimes 1: \mathfrak{l} \longrightarrow \overline{\mathrm{Cl}}(\mathfrak{l})^{0} \otimes R, \quad \ell^{-1}: \mathfrak{l}[1] \hookrightarrow \overline{\mathrm{Cl}}(\mathfrak{l})^{-1} \longrightarrow \overline{\mathrm{Cl}}(\mathfrak{l}) \otimes R .
$$

Then $\ell$ is a morphism of graded Lie algebras.

(3) We define the following elements.

- $\widetilde{\mu} \in \mathfrak{l}^{*} \otimes R \subset(\overline{\mathrm{Cl}}(\mathfrak{l}) \otimes R)^{1}$ is the element corresponding to $\mu$.

- $\beta^{\prime} \in \mathfrak{l}^{*} \otimes \overline{\mathrm{Cl}}(\mathfrak{l})^{0}$ is the element corresponding to $\beta$.

- $\beta^{\prime \prime} \in \overline{\mathrm{Cl}}(\mathfrak{l})^{1}$ is the image of $\beta^{\prime}$ by the product map $\mathfrak{l}^{*}[-1] \otimes \overline{\mathrm{Cl}}(\mathfrak{l}) \rightarrow \overline{\mathrm{Cl}}(\mathfrak{l})$.

- $\widetilde{\beta} \in(\overline{\mathrm{Cl}}(\mathfrak{l}) \otimes R)^{1}$ is the image of $\beta^{\prime \prime}$ by $\overline{\mathrm{Cl}}(\mathfrak{l}) \rightarrow \overline{\mathrm{Cl}}(\mathfrak{l}) \otimes R$.

Then the classical BRST charge

$$
\bar{Q}:=\widetilde{\mu}+\frac{1}{2} \widetilde{\beta} \in(\overline{\mathrm{Cl}}(\mathfrak{l}) \otimes R)^{1}
$$

satisfies $\{\bar{Q}, \bar{Q}\}=0$, where $\{-,-\}$ denotes the Poisson bracket of the tensor product $\overline{\mathrm{Cl}}(\mathfrak{l}) \otimes R$ of graded Poisson algebras.

Now we can introduce: 
Definition 2.2.3. The classical BRST complex $\operatorname{BRST}_{\mathrm{cl}}(\mathfrak{l}, R, \mu)$ is the dg Poisson algebra which is the tensor product

$$
\operatorname{BRST}_{\mathrm{cl}}(\mathfrak{l}, R, \mu):=\overline{\mathrm{Cl}}(\mathfrak{l}) \otimes R
$$

as a graded Poisson algebra and the differential is given by $d_{\mathrm{cl}}:=d_{\overline{\mathrm{Cl}}(\mathfrak{r}) \otimes R}+\{\bar{Q},-\}$, where the first term is the differential of the tensor product of complexes. The cohomology is denoted by

$$
H_{\mathrm{cl}}^{\frac{\infty}{2}+\bullet}(\mathfrak{l}, R, \mu):=H^{\bullet} \operatorname{BRST}_{\mathrm{cl}}(\mathfrak{l}, R, \mu),
$$

which is a graded Poisson algebra.

Let us recall some properties of the classical BRST complex following [BD, 1.4.26]. We omit the proofs.

Lemma 2.2.4. Let $(\mathfrak{l}, R, \mu)$ be the same as in Lemma 2.2.2. We also use the symbol $\ell$ there.

(1) $\ell: \mathfrak{l}_{\dagger} \rightarrow \overline{\mathrm{Cl}}(\mathfrak{l}) \otimes R$ is a morphism of $\mathrm{dg}$ Lie algebras.

(2) We have $\left[\bar{Q}, \ell^{-1}\right]=\ell^{0}$.

(3) Let $J$ be the dg ideal of $\operatorname{BRST}_{\mathrm{cl}}(\mathfrak{l}, R, \mu)$ generated by $\bar{\psi}_{i}^{*}$ 's. Then the morphism $\operatorname{Sym}\left(\mathfrak{l}^{*}[-1]\right) \otimes R \rightarrow$ $\overline{\mathrm{Cl}}(\mathfrak{l}) \otimes R / J$ of graded algebras is surjective, and the kernel is the ideal generated by $\mu(\mathfrak{l})$.

(4) Denoting $\bar{R}:=R / \mu(\mathfrak{l}) R$, we have $\operatorname{BRST}_{\mathrm{cl}}(\mathfrak{l}, R, \mu) / J \simeq \bar{R} \otimes \operatorname{Sym}\left(\mathfrak{l}^{*}[-1]\right)$ as cdgas.

(5) The differential on $\mathrm{BRST}_{\mathrm{cl}}(\mathfrak{l}, R, \mu) / J$ induced by $d_{\mathrm{cl}}$ coincides with the Chevalley-Eilenberg differential on $\bar{R} \otimes \operatorname{Sym}\left(\mathfrak{l}^{*}[-1]\right) \simeq \mathrm{CE}(\mathfrak{l}, \bar{R})$, where we regard $\bar{R}$ as a l-module in terms of the action induced by $\mu$ and the Poisson bracket. Thus we have the surjection of cdgas

$$
\operatorname{BRST}_{\mathrm{cl}}(\mathfrak{l}, R, \mu) \longrightarrow \mathrm{CE}(\mathfrak{l}, \bar{R}) .
$$

(6) On the zero-th cohomology we have a morphism $H_{\mathrm{cl}}^{\frac{\infty}{2}+0}(\mathfrak{l}, R, \mu) \rightarrow \bar{R}^{\mathfrak{l}}$ of commutative algebras, where $\bar{R}^{\mathrm{l}}$ denotes the l-invariant part of $\bar{R}$. Moreover, it is a morphism of Poisson algebras, where $\bar{R}^{\mathrm{l}}$ is regarded as a Poisson algebra with the induced Poisson structure from $R$.

The following lemma can be checked by direct computation. It recovers the original definition by Kostant and Sternberg [KS87] in the finite-dimensional case.

Lemma 2.2.5. Assume that the dg Lie algebra $\mathfrak{l}$ is of finite dimension. Choose a linear basis $\left\{x_{i} \mid i \in I\right\}$ of $\mathfrak{l}$, and let $\bar{\psi}_{i} \in \mathfrak{l}[1] \subset \overline{\mathrm{Cl}}(\mathfrak{l}), \bar{\psi}_{i}^{*} \in \mathfrak{l}^{*}[-1] \subset \overline{\mathrm{Cl}}(\mathfrak{l})$ be as before.

(1) The classical BRST charge is expressed as

$$
\bar{Q}=\sum_{i \in I} \mu\left(x_{i}\right) \otimes \bar{\psi}_{i}^{*}-\frac{1}{2} \sum_{i, j, k \in I} c_{i j}^{k} \otimes \bar{\psi}_{i}^{*} \bar{\psi}_{j}^{*} \bar{\psi}_{k} .
$$

Here $c_{i j}^{k}$ denotes the structure constant of $\mathfrak{r}:\left[x_{i}, x_{j}\right]=\sum_{i, j, k \in I} c_{i j}^{k} x_{k}$.

(2) For $r \in R$ we have $\{\bar{Q}, r \otimes 1\}=\sum_{i}\left\{\mu\left(x_{i}\right), r\right\}_{R} \otimes \bar{\psi}_{i}^{*}$.

(3) For $\eta=\sum_{k} \eta_{k} \bar{\psi}_{k}^{*} \in \Lambda^{1}\left(\mathfrak{l}^{*}\right)$ we have $\{\bar{Q}, 1 \otimes \eta\}=-\frac{1}{2} \sum_{i, j, k} \eta_{k} c_{i j}^{k} \bar{\psi}_{i}^{*} \bar{\psi}_{j}^{*}$,

(4) For $y=\sum_{i} y_{i} \bar{\psi}_{i} \in \bigwedge^{1}(\mathfrak{l})$ we have $\{\bar{Q}, 1 \otimes y\}=\sum_{i} y_{i} \mu\left(x_{i}\right)+\sum_{i, j, k} c_{i j}^{k} y_{j} \bar{\psi}_{i}^{*} \bar{\psi}_{k}$,

Let us also recall the relation between the classical BRST complex and the Koszul complex.

Definition 2.2.6. Let $\mathfrak{l}, R, \mu$ be as in Definition 2.2.2.

(1) The Koszul cdga is the cdga

$$
\operatorname{Kos}(\mathfrak{l}, R, \mu):=(\operatorname{Sym}(\mathfrak{l}[1]) \otimes R, d, \cdot)
$$

of which

- the commutative graded algebra structure $(\operatorname{Sym}(\mathfrak{l}[1]) \otimes R, \cdot)$ is given by the tensor product of the graded algebras $\operatorname{Sym}(\mathfrak{r}[1])$ and $R$ (Definition 1.2.3 with forgetting the differentials), and

- the differential is $d=d_{\mathrm{Sym}(\mathfrak{r}) \otimes R}+d_{\mathrm{Kos}}$, where $d_{\mathrm{Sym}(\mathfrak{r}) \otimes R}$ is the tensor product differential (Definition 1.1.4), and $d_{\text {Kos }}$ is the Koszul differential given by

$$
d_{\text {Kos }}\left(x_{1} \wedge \cdots \wedge x_{p} \otimes r\right)=\sum_{i=1}^{p}(-1)^{\sum_{a=1}^{i-1}\left(\left|x_{a}\right|+1\right)+\left|x_{i}\right|+\left|x_{i}\right| \sum_{a=i+1}^{p}\left(\left|x_{a}\right|+1\right)} x_{1} \wedge \cdots \widehat{x_{i}} \cdots \wedge x_{p} \otimes \mu\left(x_{i}\right) r .
$$

It is a commutative ring object in $\mathfrak{l}$-dgMod.

(2) Assume that $\mathfrak{l}$ is finite dimensional. We define the dg Poisson algebra

$$
\operatorname{BRST}_{\mathrm{cl}}^{\prime}(\mathfrak{l}, R, \mu)
$$

as follows. 
- The underlying cdga is the Chevalley-Eilenberg cdga

$$
\operatorname{CE}(\mathfrak{l}, \operatorname{Kos}(\mathfrak{l}, R, \mu))=\underline{\operatorname{Hom}}(\operatorname{Sym}(\mathfrak{l}[1]), \operatorname{Sym}(\mathfrak{l}[1]) \otimes R),
$$

which is isomorphic to

$$
\operatorname{Sym}\left(\mathfrak{l}^{*}[-1] \oplus \mathfrak{l}[1]\right) \otimes R
$$

under the assumption on $\mathfrak{l}$.

- The underlying graded Lie algebra is the tensor product $\operatorname{Sym}\left(\mathfrak{I}^{*}[-1] \oplus \mathfrak{l}[1]\right) \otimes R$ whose Lie bracket $\{-,-\}$ is given by the Leibniz rule and

$$
\{x, y\}=\{f, g\}=0, \quad\{f, x\}=f(x) \quad\left(x, y \in \mathfrak{l}, f, g \in \mathfrak{l}^{*}\right) .
$$

Remark. In (2) the differential of the Chevalley-Eilenberg dga is indeed a derivation with respect to the Lie bracket due to the momentum map equation (1.3).

Using the explicit form of the classical BRST charge (Lemma 2.2.5), we can check:

Lemma. Let $\mathfrak{l}, R, \mu$ be as in Definition 2.2.6 (2). Then we have an isomorphism of dg Poisson algebras

$$
\operatorname{BRST}_{\mathrm{cl}}^{\prime}(\mathfrak{l}, R, \mu) \simeq \operatorname{BRST}_{\mathrm{cl}}(\mathfrak{l}, R, \mu) .
$$

Now we explain the work of Safronov [S17, §2.3]. Let $(\mathfrak{l}, R, \mu)$ be as above. Then we have the Poisson reduction $\mathrm{CE}(\mathfrak{l}, \mathbb{k}) \otimes_{\mathrm{CE}(\mathfrak{l}, \operatorname{Sym} \mathfrak{l})}^{\mathbb{L}} \mathrm{CE}(\mathfrak{l}, R)$. (Definition 2.1.12).

Fact 2.2.7 ([S17, Corollary 2.7]). The derived Hamiltonian reduction $R / /{ }_{\mu}^{\mathbb{L}} \operatorname{Sym}(\mathfrak{l})=\mathrm{CE}(\mathfrak{l}, \mathbb{k}) \otimes_{\mathrm{CE}(\mathfrak{l}, \operatorname{Sym} \mathfrak{l})}^{\mathbb{L}}$ $\mathrm{CE}(\mathfrak{l}, R)$ (Definition 2.1.12) is quasi-isomorphic to the classical BRST complex as cdgas:

$$
R / /{ }_{\mu}^{\mathbb{L}} \operatorname{Sym}(\mathfrak{l}) \underset{\text { qis }}{\simeq} \operatorname{BRST}_{\mathrm{cl}}(\mathfrak{l}, R, \mu)
$$

For later use, we copy the proof in loc. cit.

Proof. We will construct the following quasi-isomorphisms of cdgas:

$$
\operatorname{BRST}_{\mathrm{cl}}(\mathfrak{l}, R, \mu) \underset{\text { qis }}{\stackrel{\sim}{\longrightarrow}} \mathrm{CE}\left(\mathfrak{l}, \mathbb{k} \otimes_{\operatorname{Sym}(\mathfrak{l})}^{\mathbb{L}} R\right) \underset{\text { qis }}{\stackrel{\sim}{\longrightarrow}} R / /{ }_{\mu}^{\mathbb{L}} \operatorname{Sym}(\mathfrak{l}) .
$$

Let us build the first quasi-isomorphism. We have the following quasi-isomorphism of $\mathfrak{l}$-modules:

$$
\operatorname{Sym}(\mathfrak{l}[1]) \otimes R \underset{\text { qis }}{\stackrel{\sim}{\longrightarrow}} \mathbb{k} \otimes_{\text {Sym } \mathfrak{l}}^{\mathbb{L}} R, \quad x_{1} \wedge \cdots \wedge x_{p} \otimes r \longmapsto \sum_{\sigma \in \Sigma_{p}}(-1)^{\varepsilon}\left[x_{\sigma(1)}|\cdots| x_{\sigma(p)} \mid r\right] .
$$

Here we used the notation for the two-sided bar complex in Definition 2.1.8, and the signature $\varepsilon$ comes from the braiding isomorphisms for the permutation $\sigma$ of $x_{i}$ 's. By the functoriality of $\mathrm{CE}(\mathfrak{l},-)$, we have the desired quasi-isomorphism of cdgas:

$$
\operatorname{BRST}_{\mathrm{cl}}(\mathfrak{l}, R, \mu) \underset{\text { qis }}{\stackrel{\sim}{\longrightarrow}} \mathrm{CE}\left(\mathfrak{l}, \mathbb{k} \otimes_{\text {Sym }(\mathfrak{l})}^{\mathbb{L}} R\right) .
$$

Next we consider the second quasi-isomorphism. As we argued in Definition 2.2.6 (2), for a l-module $M$ we have an isomorphism

$$
\mathrm{CE}(\mathfrak{l}, M)=\underline{\operatorname{Hom}}(\operatorname{Sym}(\mathfrak{r}[1]), M) \simeq \operatorname{Sym}\left(\mathfrak{l}^{*}[-1]\right) \otimes M
$$

of cdgas since $\mathfrak{l}$ is finite dimensional. Then we have

$$
R / /_{\mu}^{\mathbb{L}} \operatorname{Sym}(\mathfrak{l})=\mathrm{CE}(\mathfrak{l}, \mathbb{k}) \otimes_{\mathrm{CE}(\mathfrak{l}, \operatorname{Sym}(\mathfrak{l}))}^{\mathbb{L}} \mathrm{CE}(\mathfrak{l}, R) \simeq V \otimes W
$$

as cdgas, where we set

$$
V:=\mathbb{k} \otimes_{\operatorname{Sym}(\mathfrak{l})}^{\mathbb{L}} R, \quad W:=\operatorname{Sym}\left(\mathfrak{l}^{*}[-1]\right) \otimes_{\operatorname{Sym}\left(\mathfrak{l}^{*}[-1]\right)}^{\mathbb{L}} \operatorname{Sym}\left(\mathfrak{l}^{*}[-1]\right) .
$$

Recall that the derived tensor product is presented by the two-sided bar complex (Definition 2.1.8): $A \otimes_{B}^{\mathbb{L}} C=$ $A \otimes T(B) \otimes C$, where $T(-)$ denotes the tensor algebra of a dg algebra (Example 1.2.2 (1)). Let us denote $W^{\prime}:=\operatorname{Sym}\left(\mathfrak{l}^{*}[-1]\right)$. Then the multiplication map gives $W \simeq W^{\prime} \otimes T\left(W^{\prime}\right) \otimes W^{\prime} \rightarrow W^{\prime}$. It has a splitting $W^{\prime} \rightarrow W^{\prime} \otimes T\left(W^{\prime}\right) \otimes W^{\prime}, x \mapsto x \otimes 1 \otimes 1$. Thus we have a quasi-isomorphism $V \otimes W^{\prime} \underset{\text { qis }}{\stackrel{\sim}{\longrightarrow}} V \otimes W$ of cdgas, which yields the desired quasi-isomorphism of cdgas:

$$
R / /{ }_{\mu}^{\mathbb{L}} \operatorname{Sym}(\mathfrak{l}) \underset{\text { qis }}{\sim} V \otimes W^{\prime} \simeq \mathrm{CE}\left(\mathfrak{l}, \mathbb{k} \otimes_{\operatorname{Sym}(\mathfrak{l})}^{\mathbb{L}} R\right) .
$$


Recall that $R / / \mathbb{L}_{\mu}^{\mathbb{L}} \operatorname{Sym}(\mathfrak{l})$ is a homotopy Poisson algebra, and $\operatorname{BRST}_{\mathrm{cl}}(\mathfrak{l}, R, \mu)$ is a dg Poisson algebra. The following statement is a slight generalization of [S17, Remark 2.8]:

Proposition 2.2.8. Let $\mathfrak{l}$ be a finite-dimensional Lie algebra, and $R$ be a $\mathrm{dg}$ Poisson algebra with Hamiltonian $\mathfrak{g}$-action. Then the quasi-isomorphism $R / / \mathbb{L}_{\mu}^{\mathbb{L}} \operatorname{Sym}(\mathfrak{g}) \underset{\text { qis }}{\simeq} \operatorname{BRST}_{\mathrm{cl}}(\mathfrak{g}, R, \mu)$ in Fact 2.2 .7 gives an equivalence as homotopy Poisson algebras.

Proof. It is enough to check that the composition (2.3) respects the Poisson bracket $\{\cdot, \cdot \cdot\}_{\text {BRST }}$ on the classical BRST complex $\operatorname{BRST}_{\mathrm{cl}}(\mathfrak{g}, R, \mu)$ and the map $l_{2}$ of the $L_{\infty}$-structure on the derived Hamiltonian reduction $R / / \mathbb{L}_{\mu}^{\mathbb{L}} \operatorname{Sym}(\mathfrak{g})$.

Recall that we have

$$
\operatorname{BRST}_{\mathrm{cl}}(\mathfrak{g}, R, \mu)=\overline{\mathrm{Cl}}(\mathfrak{g}) \otimes R
$$

as graded Poisson algebras, where $\overline{\mathrm{Cl}}(\mathfrak{g})$ denotes the classical Clifford algebra, and the Poisson bracket can be written as $\{\cdot, \cdot\}_{\mathrm{BRST}}=\{\cdot, \cdot\}_{\overline{\mathrm{Cl}}} \cdot M+\cdot \overline{\mathrm{Cl}}\{\cdot, \cdot\}_{\mathrm{BRST}}$.

On the $L_{\infty}$-structure of $R / / \mathbb{L}_{\mu}^{\mathbb{L}} \operatorname{Sym}(\mathfrak{g})$, let us unwind the proof of Fact 2.1.7 explained in $§ 2.1 .2$. Denoting

$$
A:=\mathrm{CE}(\mathfrak{g}, \operatorname{Sym}(\mathfrak{g})), \widetilde{A}:=T(A[1]), L:=\mathrm{CE}(\mathfrak{g}, \mathbb{k}), \widetilde{L}:=L \otimes \widetilde{A}, M:=\mathrm{CE}(\mathfrak{g}, R), \widetilde{M}:=\widetilde{A} \otimes M,
$$

we have

$$
R / /{ }_{\mu}^{\mathbb{L}} \operatorname{Sym}(\mathfrak{g}) \simeq \operatorname{Eq}(\widetilde{L} \otimes \widetilde{M} \rightrightarrows \widetilde{L} \otimes \widetilde{A} \otimes \widetilde{M}),
$$

and the $L_{\infty}$-structure comes from that on $\widetilde{L} \otimes \widetilde{M}$. It is enough to compute $l_{2}$ on $\widetilde{L}$ and $\widetilde{M}$. We only argue that on $\widetilde{M}=\widetilde{A} \otimes M$, which is given by $(2.2)$.

The operation coming from $M$ is $\{\cdot, \cdot\}_{M}$, which is obviously compatible with the part of $\{\cdot, \cdot\}_{\text {BRST }}$. Unwinding the definitions, the operation coming from $\widetilde{A}$ is given by the first line of $(2.2)$ with $p=1, f_{1}$ : $A \rightarrow \mathfrak{g}^{*}[-1] \oplus \mathbb{T}_{R}[-1] \oplus \mathfrak{g}$, which is the first component of the lifting $A \rightarrow Z(M)$ of the coisotropic morphism $\mathrm{CE}(\mathfrak{g}, \mu): A \rightarrow M$. Under the identification $A=\mathrm{CE}(\mathfrak{g}, \operatorname{Sym}(\mathfrak{g})) \simeq S^{*} \otimes \operatorname{Sym}(\mathfrak{g})$ with $S^{*}:=\operatorname{Sym}\left(\mathfrak{g}^{*}[-1]\right)$, the map $f_{1}$ is determined by the image of $S^{*}$ and $\mathfrak{g}$. Recall the description of $f_{1}$ from Fact 2.1.11, we have $f_{1}(a \otimes 1)=\operatorname{pr}_{1}(a)$ for $a \in S^{*}, \operatorname{pr}_{1}: S^{*} \rightarrow \mathfrak{g}^{*}[-1]$, and $f_{1}(1 \otimes x)=\mu(x)-x$ for $x \in \mathfrak{g}$. Using this description, we can check that under the composition (2.3) the two Poisson structures are compatible.

For later use, we also give a infinite-dimensional modification of Proposition 2.2.8.

Definition 2.2.9. Let $V$ be a linear space equipped with a decomposition $V=\bigoplus_{\gamma \in \Gamma} V_{\gamma}$ by an abelian group $\Gamma$ such that each component $V_{\gamma}$ is finite-dimensional.

(1) We call such a decomposition a finite-dimensional $\Gamma$-decomposition.

(2) We set $V^{\vee}:=\bigoplus_{\gamma \in \Gamma} V_{\gamma}^{*}$ and call it the restricted dual of $V$.

(3) For another linear space $W$, we denote $\operatorname{Hom}^{\mathrm{rst}}(V, W):=\bigoplus_{\gamma \in \Gamma} \operatorname{Hom}\left(V_{\gamma}, W\right) \simeq V^{\vee} \otimes W$.

Proposition 2.2.10. Let $\mathfrak{g}$ be a Lie algebra, and $R$ be a Poisson algebra with a Hamiltonian $\mathfrak{g}$-action. Assume that $\mathfrak{g}$ has a finite-dimensional $\Gamma$-decomposition $\mathfrak{g}=\bigoplus_{\gamma \in \Gamma} \mathfrak{g}_{\gamma}$ and the $\mathfrak{g}$-action on $M$ is locally finite. Then replacing Hom by Hom rst in the definition of the Chevalley-Eilenberg complexes, we have an quasi-isomorphism

$$
R / /{ }_{\mu}^{\mathbb{L}} \operatorname{Sym}(\mathfrak{g}) \underset{\text { qis }}{\simeq} \operatorname{BRST}_{\mathrm{cl}}(\mathfrak{g}, R, \mu)
$$

of homotopy Poisson algebras.

Proof. In the proof of Fact 2.2.7 (and that of Proposition 2.2.8), we used the finite-dimensional assumption only at the isomorphism $\operatorname{CE}(\mathfrak{g},-)=\underline{\operatorname{Hom}}(\operatorname{Sym}(\mathfrak{g}[1]),-) \simeq \operatorname{Sym}^{*}(\mathfrak{g}[-1]) \otimes-$ for the Chevalley-Eilenberg complex. Thus under the assumption and the replacement we can apply the same argument, and have the consequence from Proposition 2.2.8.

Remark 2.2.11. Let us give a connection to the argument in $[A, \S 3$, p. 8]. For simplicity we set $\mathbb{k}:=\mathbb{C}$. We make the following assumptions:

(i) $\mathfrak{g}$ is the Lie algebra of a linear algebraic group $G$.

(ii) $R$ is a Poisson algebra equipped with Hamiltonian $\mathfrak{g}$-action. We denote $X:=\operatorname{Spec}(R)$ and consider it as an affine Poisson scheme with Hamiltonian $G$-action.

(iii) The momentum map $\mu_{X}: X \rightarrow \mathfrak{g}^{*}$ corresponding to $\mu_{R}$ is flat as a morphism of schemes. 
Then we have the non-derived Hamiltonian reduction $X / / G$, which is isomorphic to $\mu^{-1}(0) / G$ as a scheme. On the other hand, from Proposition 2.2.8 we have an isomorphism

$$
\operatorname{BRST}_{\mathrm{cl}}(\mathfrak{g}, R, \mu) \simeq R / /{ }_{\mu} \operatorname{Sym}(\mathfrak{g})=\mathbb{C}\left[\mu^{-1}(0) / G\right]
$$

of graded Poisson algebras. Here the symbol $/ / \mu$ in the middle is the non-derived Hamiltonian reduction of Poisson algebra. Thus, taking cohomology, we have

$$
H^{\bullet} \operatorname{BRST}_{\mathrm{cl}}(\mathfrak{g}, R, \mu) \simeq H^{\bullet} \mathbb{C}\left[\mu^{-1}(0) / G\right] \simeq \mathbb{C}\left[\mu^{-1}(0)\right] \otimes H^{\bullet}(G, \mathbb{C})
$$

and recover the computation of the classical BRST cohomology in [Ku15].

2.3. Moore-Tachikawa varieties in derived setting. The main purpose of this subsection is to recall the proposal of Calaque [C14] which gives a derived geometric approach to Moore-Tachikawa varieties. We make a slight modification and treat affine derived schemes only.

We work over $\mathbb{C}$ in this subsection. As in the latter half of $\S 1.3 .3$, we consider simply connected semisimple algebraic groups over $\mathbb{C}$. We identify such a group $G$ and its Lie algebra $\mathfrak{g}:=\operatorname{Lie}(G)$. Recall Definition 1.3.11 of Hamiltonian $\mathfrak{g}$-action and that we denote by $R^{\mathrm{op}}$ the opposite algebra of a $\widehat{\mathbb{P}}_{n}$-algebra $R$ (Definition 2.1.5 (2)).

Definition 2.3.1 (c.f. [C14, Example 3.5]). We define the category MT of derived Moore-Tachikawa varieties by the following description.

- An object is a simply connected semi-simple algebraic group $G$ over $\mathbb{C}$. We identify it with the associated Lie algebra $\mathfrak{g}$.

- A morphisms from $\mathfrak{g}_{1}$ to $\mathfrak{g}_{2}$ is an equivalence classes of $\widehat{\mathbb{P}}_{1}$-algebras $R$ in dgVec together with Hamiltonian $\left(\mathfrak{g}_{1} \oplus \mathfrak{g}_{2}\right)$-action. The momentum map is denoted by $\mu_{R}=\mu_{R}^{1}+\mu_{R}^{2}: \mathfrak{g}_{1} \oplus \mathfrak{g}_{2} \rightarrow R$.

- The composition of $R \in \operatorname{Hom}_{\mathrm{MT}}\left(\mathfrak{g}_{1}, \mathfrak{g}_{2}\right)$ and $R^{\prime} \in \operatorname{Hom} \operatorname{MT}\left(\mathfrak{g}_{2}, \mathfrak{g}_{3}\right)$ is given by

$$
R^{\prime} \widetilde{\circ} R:=\left(R^{\mathrm{op}} \otimes R^{\prime}\right) /{ }_{\mu}^{\mathbb{L}} \operatorname{Sym}\left(\mathfrak{g}_{2}\right)
$$

where the momentum map $\mu: \mathfrak{g}_{2} \rightarrow R^{\mathrm{op}} \otimes R^{\prime}$ is given by $\mu:=-\mu_{R}^{2} \otimes 1+1 \otimes \mu_{R^{\prime}}^{1}$. We call $R^{\prime} \widetilde{\circ} R$ the (derived) gluing.

We denote by $\mathrm{MT}^{\text {nd }} \subset \mathrm{MT}$ the full subcategory spanned by non-degenerate $\widehat{\mathbb{P}}_{1}$-algebras $R$ (Definition 1.4.6).

Remark 2.3.2. (1) The gluing $R^{\prime} \widetilde{\circ} R$ has a Hamiltonian $\left(\mathfrak{g}_{1} \oplus \mathfrak{g}_{3}\right)$-action with the momentum map $-\mu_{R}^{1} \otimes 1+1 \otimes \mu_{R^{\prime}}^{2}$. Thus $\widetilde{o}$ is well-defined. The associativity of composition holds since $/ / \mathbb{L}_{\mu}$ is realized as a pushforward in the $\infty$-category dguCom. Note also that if $R$ and $R^{\prime}$ is non-degenerate, then $R^{\prime} \widetilde{\circ} R$ is also non-degenerate by Lemma 2.1.13 (2). Thus $\mathrm{MT}^{\text {nd }}$ is surely a full subcategory.

(2) As we recalled in $§ 0.1 .1$, the original proposal of Moore and Tachikawa in [MT12] is given in terms of (non-derived) holomorphic symplectic varieties with Hamiltonian actions and an additional $\mathbb{C}^{*}$ action.

(3) We explain the definition of the category by Calaque [C14, Example 3.5]. Objects are the same, and a morphism from $G_{1}$ to $G_{2}$ is a derived 0 -symplectic scheme $\left(X, \omega_{X}\right)$ equipped with Hamiltonian $\left(G_{1} \times G_{2}\right)$-action. We denote by $\mu_{X}: X \rightarrow \mathfrak{g}^{*}$ the corresponding momentum map. The composition of $X \in \operatorname{Hom}\left(G_{1}, G_{2}\right)$ and $Y \in \operatorname{Hom}\left(G_{2}, G_{3}\right)$ is given by

$$
\left[\left(\left(X^{\mathrm{op}} \times Y\right) / /^{\mathbb{L}} \Delta\left(G_{2}\right)\right) /\left(G_{1} \times G_{3}\right)\right] \simeq\left[X^{\mathrm{op}} /\left(G_{1} \times G_{2}\right)\right] \times_{\left[\mathfrak{g}_{2}^{*} / G_{2}\right]}^{\mathbb{L}}\left[Y /\left(G_{2} \times G_{3}\right)\right]
$$

with the following notations:

- $X^{\text {op }}$ denotes the derived scheme $X$ with the opposite 0 -symplectic structure $-\omega_{X}$.

- For a derived scheme $Z$ with $G$-action, the symbol $[Z / G]$ denotes the quotient derived stack.

- In the left hand side $\left(X^{\mathrm{op}} \times Y\right) / /^{\mathbb{L}} \Delta\left(G_{2}\right)$ denotes the derived symplectic reduction [C14, Example $3.4]$ with respect to the diagonal $G_{2}$-action and the momentum map $-\mu_{X}+\mu_{Y}: X^{\mathrm{op}} \times Y \rightarrow \mathfrak{g}_{2}^{*}$.

- The right hand side denotes the derived Lagrangian intersection [C14, Example 3.2].

We can partially recover Calaque's definition from $\mathrm{MT}^{\text {nd }}$ in an easy manner. By Definition 1.4.6 (3) of shifted symplectic structure, we can restate that a morphism in MT is a cdga equipped with a 0-symplectic structure and a Hamiltonian action. Adding the condition that all the cdgas are concentrated in non-positive cohomological degree, we recover the description in (3) by translating the construction on cdgas to affine derived schemes. 
2.4. The case of $G$-equivariant Poisson algebras. In this subsection we translate the arguments on classical BRST reduction discussed in the beginning of [A, §3] into our language. We work over $\mathbb{C}$ here.

Let $G$ be a simply connected semisimple algebraic group. We denote by $\mathfrak{g}:=\operatorname{Lie}(G)$ the Lie algebra of $G$. We regard the linear dual $\mathfrak{g}^{*}$ as an affine scheme with the coordinate ring $\mathbb{C}\left[\mathfrak{g}^{*}\right]=\operatorname{Sym}(\mathfrak{g})$, so that the Lie-Poisson algebra structure makes $\mathfrak{g}^{*}$ an affine Poisson scheme.

For the Lie-Poisson algebra $\operatorname{Sym}(\mathfrak{g})$, a Poisson $\operatorname{Sym}(\mathfrak{g})$-module $M$ in $\operatorname{Vec}$ (Definition 1.3.3) is nothing but a $\operatorname{Sym}(\mathfrak{g})$-module together with a morphism ad $: \mathfrak{g} \rightarrow \operatorname{End}(M)$ of Lie algebras such that ad $(x)(s . m)=$ $\{x, s\} \cdot m+s \cdot \operatorname{ad}(x)(m)$ for any $x \in \mathfrak{g}, s \in \operatorname{Sym}(\mathfrak{g})$ and $m \in M$. We can recast such a Poisson module in terms of sheaves over the affine Poisson scheme $\mathfrak{g}^{*}$.

Notation 2.4.1. Let us consider a scheme $X$ over $\mathbb{C}$.

(1) If $X$ is a $G$-scheme, then we denote by $\mathrm{QCoh}^{G}(X)$ the category of $G$-equivariant quasi-coherent sheaves of $\mathcal{O}_{X}$-modules.

(2) If $X$ is a Poisson scheme, then we denote by PoisQCoh $(X)$ the category of sheaves on $X$ which are both quasi-coherent sheaves of $\mathcal{O}_{X}$-modules and sheaves of Lie algebra $\mathcal{O}_{X}$-modules whose local sections satisfy the relation in Definition 1.3.3 (iii).

Regarding $\mathfrak{g}^{*}$ as a $G$-scheme by the coadjoint action, we find that an object of $\mathrm{QCoh}{ }^{G}\left(\mathfrak{g}^{*}\right)$ has a structure of a sheaf of Lie algebra $\mathcal{O}_{\mathfrak{g}^{*}}$-modules induced by the coadjoint action of $G$. Thus we have an embedding

$$
\mathrm{QCoh}{ }^{G}\left(\mathfrak{g}^{*}\right) \longleftrightarrow \text { PoisQCoh }\left(\mathfrak{g}^{*}\right) \text {. }
$$

On the other hand, since $\mathfrak{g}^{*}=\operatorname{Spec}\left(\mathbb{C}\left[\mathfrak{g}^{*}\right]\right)=\operatorname{Spec}(\operatorname{Sym}(\mathfrak{g}))$ is an affine Poisson scheme, we have an equivalence of categories

$$
\text { PoisQCoh }\left(\mathfrak{g}^{*}\right) \stackrel{\sim}{\longrightarrow} \operatorname{Sym}(\mathfrak{g}) \text {-PMod. }
$$

For a given $\mathcal{M} \in \mathrm{Q} \operatorname{Coh}^{G}\left(\mathfrak{g}^{*}\right)$, let us denote by $M$ the corresponding Poisson $\operatorname{Sym}(\mathfrak{g})$-module under the composition $Q \operatorname{Coh}^{G}\left(\mathfrak{g}^{*}\right) \hookrightarrow \operatorname{Pois} Q \operatorname{Coh}\left(\mathfrak{g}^{*}\right) \simeq \operatorname{Sym}(\mathfrak{g})$-PMod. Then we can see that the Lie algebra morphism ad $: \mathfrak{g} \rightarrow \operatorname{End}(M)$ corresponds to the Lie algebra action of $\mathcal{O}_{\mathfrak{g}^{*}}$ on $\mathcal{M}$ induced by the $G$-action. In total, we have:

Lemma 2.4.2. We have an equivalence of categories

$$
\mathrm{QCoh}{ }^{G}\left(\mathfrak{g}^{*}\right) \simeq \operatorname{Sym}(\mathfrak{g})-\mathrm{PMod}^{\mathrm{lf}},
$$

where $\operatorname{Sym}(\mathfrak{g})-$ PMod $^{\text {lf }}$ denotes the full subcategory of $\operatorname{Sym}(\mathfrak{g})$-PMod spanned by those objects on which the adjoint action of $\mathfrak{g}$ is locally finite.

The category $\mathrm{QCoh}{ }^{G}\left(\mathfrak{g}^{*}\right)$ is a symmetric monoidal category by the tensor product $\otimes_{\mathcal{O}_{\mathfrak{g}}^{*}}$, so that we have the notion of Poisson algebra objects therein. Using the equivalence in Lemma 2.4.2 and unwinding the definition, we recover the claim in $[\mathrm{A}, \S 3$, p. 8$]$ :

Lemma 2.4.3. A Poisson algebra object in the symmetric monoidal category $Q \operatorname{Coh}^{G}\left(\mathfrak{g}^{*}\right)$ is a Poisson algebra $R$ equipped with a morphism $\mu_{R}: \operatorname{Sym}(\mathfrak{g}) \rightarrow R$ of Poisson algebras under which the adjoint action of $\mathfrak{g}$ is locally finite.

In particular, a Poisson algebra object $R$ is equipped with Hamiltonian $\mathfrak{g}$-action, and the morphism $\mu_{R}$ is the corresponding momentum map. Thus we can consider Hamiltonian reduction of Poisson algebra objects.

We have an obvious dg analogue of the above arguments. Recall Notation 1.4.1 (7): We denote by $\mathrm{L}_{\mathrm{QCoh}}\left(\mathfrak{g}^{*}\right)$ the $\infty$-category of quasi-coherent sheaves of $\mathcal{O}_{\mathfrak{g}^{*}}$-modules, whose homotopy category is equivalent to the derived category $\mathrm{D}_{\mathrm{Q} C o h}\left(\mathfrak{g}^{*}\right)$. It is a symmetric monoidal category under the derived tensor product $\otimes_{\mathcal{O}_{\mathfrak{g}^{*}}}^{\mathbb{L}}$. We also have the $\infty$-category $\mathrm{L}_{Q}^{G}$ Coh $\left(\mathfrak{g}^{*}\right)$ of $G$-equivariant sheaves, which is also equivalent to $\mathrm{L}_{Q \operatorname{Coh}}\left(\left[\mathfrak{g}^{*} / G\right]\right)$ of sheaves over the quotient stack $\left[\mathfrak{g}^{*} / G\right]$. The $\infty$-category $\mathrm{L}_{Q \operatorname{Coh}}^{G}\left(\mathfrak{g}^{*}\right)$ is also symmetric monoidal, and we can consider Poisson algebra objects therein.

As in the arguments for Lemma 2.4.3, a Poisson algebra object in $\mathrm{L}_{Q}^{G} \mathrm{Coh}\left(\mathfrak{g}^{*}\right)$ is nothing but a $\mathrm{dg}$ Poisson algebra $R$ equipped with a morphism $\mu_{R}: \operatorname{Sym}(\mathfrak{g}) \rightarrow R$ of dg Poisson algebras under which the adjoint action of $\mathfrak{g}$ is locally finite.

Now let us consider Hamiltonian reduction of Poisson algebra objects. We are interested in the derived gluing in the category MT, or the composition of morphisms therein (Definition 2.3.1). Thus, instead of considering the reduction of an object $R$, let us consider the reduction of tensor product $R^{\mathrm{op}} \otimes R^{\prime}$ where $R^{\text {op }}$ denotes the Poisson algebra $\left(R,-\{\cdot, \cdot\}_{R}\right)$ equipped with the momentum map $-\mu_{R}$. Then the derived Hamiltonian reduction corresponding to the composition of morphisms is described in the following way. 
Proposition 2.4.4. Let $R$ and $R^{\prime}$ be Poisson algebra objects in $\mathrm{L}_{Q \text { Coh }}^{G}\left(\mathfrak{g}^{*}\right)$. We denote by $\mu_{R}$ and $\mu_{R^{\prime}}$ the corresponding momentum maps respectively, and define $\mu:=-\mu_{R} \otimes 1+1 \otimes \mu_{R^{\prime}}: \operatorname{Sym}(\mathfrak{g}) \rightarrow R^{\mathrm{op}} \otimes R^{\prime}$. Then we have an quasi-isomorphism of homotopy Poisson algebras

$$
\operatorname{BRST}_{\mathrm{cl}}\left(\mathfrak{g}, R^{\mathrm{op}} \otimes R^{\prime}, \mu\right) \underset{\text { qis }}{\simeq} R^{\prime} \widetilde{o} R=\left(R^{\mathrm{op}} \otimes R^{\prime}\right) / /_{\mu}^{\mathbb{L}} \operatorname{Sym}(\mathfrak{g}) .
$$

Proof. This is a direct consequence of Proposition 2.2.8.

Remark 2.4.5. Let us continue Remark 2.2 .11 and give a connection to the argument in [A, §3, pp. 8-9]. We make the following assumptions on $R$ :

(i) $R$ is a Poisson algebra object in $Q \operatorname{Coh}^{G}\left(\mathfrak{g}^{*}\right)$. We denote $X:=\operatorname{Spec}(R)$, and consider $X$ as a affine Poisson scheme with Hamiltonian $G$-action.

(ii) There is a closed subscheme $S \subset X$ such that the action map gives an isomorphism $G \times S \stackrel{\sim}{\rightarrow} X$.

(iii) The momentum map $\mu_{X}: X \rightarrow \mathfrak{g}^{*}$ corresponding to $\mu_{R}$ is flat as a morphism of schemes.

Let $R^{\prime}$ be another Poisson object in $Q \operatorname{Coh}^{G}\left(\mathfrak{g}^{*}\right)$. Denoting $X^{\prime}:=\operatorname{Spec}\left(R^{\prime}\right)$ the corresponding affine Poisson scheme with the momentum map $\mu_{X^{\prime}}: X^{\prime} \rightarrow \mathfrak{g}^{*}$ and $X^{\mathrm{op}}:=\operatorname{Spec}\left(R^{\mathrm{op}}\right)$, we have the affine Poisson scheme $X^{\mathrm{op}} \times X$ with the flat momentum map $\mu\left(x, x^{\prime}\right):=-\mu_{X}(x)+\mu_{X^{\prime}}\left(x^{\prime}\right)$. Thus we have the non-derived Hamiltonian reduction $\left(X^{\mathrm{op}} \times X^{\prime}\right) / / \Delta(G)$. We also have

$$
\mu^{-1}(0) \simeq X \times_{\mu_{X}, \mathfrak{g}^{*}, \mu_{X^{\prime}}} X^{\prime} \simeq G \times\left(S \times_{\mu_{S}, \mathfrak{g}^{*}, \mu_{X^{\prime}}} X^{\prime}\right),
$$

where $\mu_{S}:=\left.\mu_{X}\right|_{S}: S \rightarrow \mathfrak{g}^{*}$ is the restriction. Thus we have

$$
\left(X^{\mathrm{op}} \times X^{\prime}\right) / / \Delta(G) \simeq \mu^{-1}(0) / G \simeq S \times_{\mathfrak{g}^{*}} X^{\prime}
$$

as schemes. On the other hand, from Proposition 2.4.4 we have an isomorphism

$$
\mathrm{BRST}_{\mathrm{cl}}\left(\mathfrak{g}, R^{\mathrm{op}} \otimes R^{\prime}, \mu\right) \simeq\left(R^{\mathrm{op}} \otimes R^{\prime}\right) / /{ }_{\mu} \operatorname{Sym}(\mathfrak{g}) \simeq \mathbb{k}\left[\left(X^{\mathrm{op}} \times X^{\prime}\right) / / \Delta(G)\right]
$$

of graded Poisson algebras. Here the symbol // $\mu$ in the middle denotes the non-derived Hamiltonian reduction of Poisson algebra. Combining these isomorphisms, we have

$$
H^{\bullet} \operatorname{BRST}_{\mathrm{cl}}\left(\mathfrak{g}, R^{\mathrm{op}} \otimes R^{\prime}, \mu\right) \simeq H^{\bullet} \mathbb{k}\left[\mu^{-1}(0) / G\right] \simeq \mathbb{k}\left[S \times_{\mathfrak{g}^{*}} X^{\prime}\right] \otimes H^{\bullet}(G, \mathbb{C}) .
$$

Thus we recover the formula $[\mathrm{A},(13)]$.

\section{Jet AND ARC SPACES IN DERIVED SETTING}

3.1. Jet and arc spaces for ordinary schemes. Let us recall the definition of jet spaces in the ordinary setting. We refer [BLR, §7.6] and [EM09] for the detail.

Let $\mathbb{k}$ be a field, and Sch be the category of schemes over $\mathbb{k}$.

Fact 3.1.1 ([BLR, $\S 7.6$, Theorem 4]). For $n \in \mathbb{N}$, there exists a functor $J_{n}:$ Sch $\rightarrow$ Sch such that such that for any $S \in$ Sch we have a functorial bijection

$$
\operatorname{Hom}_{\text {Sch }}\left(S, J_{n}(X)\right) \simeq \operatorname{Hom}_{\text {sch }}\left(S \times_{\operatorname{Spec}(\mathbb{k})} \operatorname{Spec}\left(\mathbb{k}[t] /\left(t^{n+1}\right)\right), X\right) .
$$

We call $J_{n}(X)$ the $n$-th jet space of $X$.

Corollary 3.1.2. By the defining condition as a functor of points, we have

(1) $J_{n}(X)$ is unique up to a canonical isomorphism.

(2) $J_{0}(X) \simeq X$.

(3) For $m, n \in \mathbb{N}$ with $m \geq n$, there is a morphism $\pi_{m, n}: J_{m} \rightarrow J_{n}$ of functors induced by the truncation $\mathbb{k}[t] /\left(t^{m+1}\right) \rightarrow \mathbb{k}[t] /\left(t^{n+1}\right)$. These morphisms satisfy $\pi_{n, p} \circ \pi_{m, n}=\pi_{m, p}$ for $m \geq n \geq p$, so that we have an inverse system $\left\{\pi_{m, n} \mid m, n \in \mathbb{N}, m \geq n\right\}$ on the direct set $\{\mathbb{N}, \leq\}$. In particular, for $n \in \mathbb{N}$, we have the following commutative diagram in Sch.

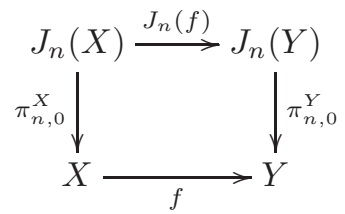

We denote by $\mathrm{Sch}^{\mathrm{ft}}$ the full subcategory of schemes of finite type over $\mathbb{k}$. We then have

Fact 3.1.3. Let $n \in \mathbb{N}$ be arbitrary.

(1) If a morphism $f$ in Sch is smooth, then so is $J_{n}(f)$ [BLR, §7.6, Proposition 5]. 
(2) $J_{n}$ gives a functor $J_{n}: \mathrm{Sch}^{\mathrm{ft}} \rightarrow \mathrm{Sch}^{\mathrm{ft}}$. In other words, for a scheme $X$ of finite type over $\mathbb{k}$, the $n$-th jet space $J_{n}(X)$ is also of finite type over $\mathbb{k}$ [BLR, $\S 7.6$, Proposition 5].

(3) For any $X \in \mathrm{Sch}^{\mathrm{ft}}$, the morphism $\pi_{n, 0}: J_{n}(X) \rightarrow J_{n, 0}(X)=X$ is affine [EM09, §2].

By [EM09, Lemma 2.9], if $f: X \rightarrow Y$ is an étale morphism in $\operatorname{Sch}^{\mathrm{ft}}$, then for every $n \in \mathbb{N}$ the commutative diagram in Corollary 3.1.2 (3) is cartesian. Using this fact, we can prove

Fact ([EM09, Lemma 2.9, Remark 2.10]). Let $f: X \rightarrow Y$ be a morphism in $\operatorname{Sch}^{\mathrm{ft}}$, and let $n \in \mathbb{N}$ be arbitrary. If $f$ is étale, then $J_{n}(f)$ is also étale.

Remark 3.1.4. Following [EM09, §3] and [AM, §3], we give an explicit description of $J_{n}(X)$ for an affine $X$. We assume the characteristics of $\mathbb{k}$ is 0 .

(1) First we assume $X=\mathbb{A}^{N}=\operatorname{Spec}\left(\mathbb{k}\left[x^{1}, \ldots, x^{N}\right]\right)$. Then we have

$$
J_{n}\left(\mathbb{A}^{N}\right)=\operatorname{Spec}\left(\mathbb{k}\left[x_{(-j-1)}^{i} \mid i=1, \ldots, N, j=0, \ldots, n\right]\right) .
$$

Indeed, for an affine scheme $S=\operatorname{Spec}(A)$, a morphism $a: \operatorname{Spec}\left(A[t] /\left(t^{n+1}\right)\right) \rightarrow \mathbb{A}^{N}$ corresponds to a morphism $a^{*}: k\left[x^{i}\right] \rightarrow A[t] /\left(t^{n+1}\right)$. We set $a^{*}\left(x^{i}\right)=\sum_{j=0}^{n} a_{(-j-1)}^{i} / j$. Then we have the morphism $\alpha^{*}: \mathbb{k}\left[x_{(-j-1)}^{i}\right] \rightarrow A,\left(x_{(-j-1)}^{i}\right) \mapsto\left(a_{(-j-1)}^{i}\right)$ corresponding to an $A$-valued point $\alpha: \operatorname{Spec}(A) \rightarrow J_{N}\left(\mathbb{A}^{N}\right)$. The correspondence $a \mapsto \alpha$ gives the desired functorial bijection

$$
\operatorname{Hom}_{\operatorname{sch}}\left(\operatorname{Spec}(A) \times_{\operatorname{Spec}(\mathbb{k})} \operatorname{Spec}\left(\mathbb{k}[t] /\left(t^{n+1}\right)\right), \mathbb{A}^{N}\right) \simeq \operatorname{Hom} \operatorname{Sch}\left(\operatorname{Spec}(A), J_{n}\left(\mathbb{A}^{N}\right)\right) .
$$

(2) Next we consider the case $X=\operatorname{Spec}(R), R=\mathbb{k}\left[x^{1}, \ldots, x^{N}\right] / I$ with $I=\left(f_{1}, \ldots, f_{M}\right)$. Define a 0 -derivation $T$ on $\mathbb{k}\left[x_{(-j-1)}^{i}\right]$ (Definition 1.2.10(3)) by $T x_{(-j)}^{i}=j x_{(-j-1)}^{i}$ for $j \in \mathbb{Z}_{>0}$. Then we have

$$
\begin{aligned}
& J_{n}(\operatorname{Spec}(R))=\operatorname{Spec}\left(J_{n}(R)\right), \\
& J_{n}(R):=\mathbb{k}\left[x_{(-j-1)}^{i} \mid i=1, \ldots, N, j=0, \ldots, n\right] /\left(T^{k} f_{l} \mid k=0, \ldots, n, l=1, \ldots, M\right) .
\end{aligned}
$$

Now we turn to the arc space. By Corollary 3.1.2 (3) and Fact 3.1.3, we have an inverse system $\left\{\pi_{m, n}\right.$ : $\left.J_{m}(X) \rightarrow J_{n}(X) \mid m, n \in \mathbb{N}, m \geq n\right\}$ of affine morphisms in $\mathrm{Sch}^{\mathrm{ft}}$. Thus the limit exists in Sch. In the affine case $X=\operatorname{Spec}(R)$, the limit is also affine.

Definition 3.1.5. (1) Let $X$ be a scheme over $\mathbb{k}$. We denote the limit of $\left\{\pi_{m, n}: J_{m}(X) \rightarrow J_{n}(X)\right\}$ in Sch by $J_{\infty}(X)$, and call it the arc space or the $\infty$-jet space of $X$. We also denote by $\psi_{n}: J_{\infty}(X) \rightarrow$ $J_{n}(X)$ the projection.

(2) For a commutative ring $R$, we denote by $J_{\infty}(R)$ the commutative ring whose spectrum gives the arc space: $\operatorname{Spec}\left(J_{\infty}(R)\right)=J_{\infty}(\operatorname{Spec}(R))$.

The properties of the $n$-jet space $J_{n}(X)$ are inherited by $J_{\infty}(X)$. For example, we have:

Lemma. (1) For $X \in \mathrm{Sch}^{\mathrm{ft}}$ and a commutative algebra $A$ over $\mathbb{k}$, there is a bijection

$$
\operatorname{Hom}_{\mathrm{Sch}}\left(\operatorname{Spec}(A), J_{\infty}(X)\right) \simeq \operatorname{Hom}_{\mathrm{Sch}}(\operatorname{Spec}(A[[t]]), X) .
$$

(2) The correspondence $X \mapsto J_{\infty}(X)$ gives a functor $\mathrm{Sch}^{\mathrm{ft}} \rightarrow$ Sch.

(3) If $f: X \rightarrow Y$ is an étale morphism, then there is a cartesian diagram

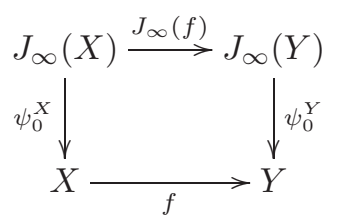

Let us consider the case when $X$ is an affine scheme of finite type over $\mathbb{k}$ of characteristics 0 . Expressing $X=\operatorname{Spec}(R)$ with $R=\mathbb{k}\left[x^{1}, \ldots, x^{N}\right] /\left(f_{1}, \ldots, f_{M}\right)$, we have $J_{\infty}(\operatorname{Spec}(R))=\operatorname{Spec}\left(J_{\infty}(R)\right)$ with

$$
J_{\infty}(R):=\mathbb{k}\left[x_{(-j-1)}^{i} \mid i=1, \ldots, N, j \in \mathbb{N}\right] /\left(T^{k} f_{l} \mid k \in \mathbb{N}, l=1, \ldots, M\right) .
$$

Note that the $\mathbb{k}$-algebra $J_{\infty}(R)$ inherits the 0 -derivation $T$ on $J_{n}\left(\mathbb{k}\left[x^{1}, \ldots, x^{N}\right]\right)$. We denote the induced 0 -derivation on $J_{\infty}(R)$ by the same symbol $T$. Then the description above yields: 
Fact 3.1.6 ([EM09, Remark 3.1]). For a commutative algebra $R$ of finite type, we have a morphism $j$ : $R \rightarrow J_{\infty}(R)$ of algebras such that given a commutative algebra $R^{\prime}$ with a 0 -derivation $T^{\prime}$ and a morphism $j^{\prime}: R \rightarrow R^{\prime}$ of algebras, there is a unique morphism $h: J_{\infty}(R) \rightarrow R^{\prime}$ of algebras making the diagram

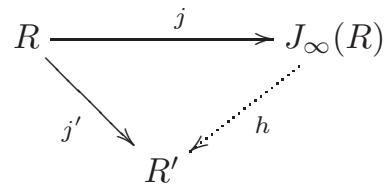

commute and satisfying $T^{\prime} h=h T$, i.e., giving a morphism $h:\left(J_{\infty}(R), T\right) \rightarrow\left(R^{\prime}, T^{\prime}\right)$ of differential algebras.

Here we used:

Definition 3.1.7. A commutative algebra equipped with a 0-derivation (Definition 1.2.10 (3)) is called a differential algebra.

Using this Fact, we can show:

Lemma 3.1.8. For a linear algebraic group $G$ over $\mathbb{k}$, the arc space is given by the proalgebraic group $J_{\infty}(G)=G[[t]]$.

3.2. Jet and arc spaces for derived schemes. Let us give a derived analogue of the previous $\S 3.1$. In this subsection we work over a field $\mathbb{k}$ containing $\mathbb{Q}$.

3.2.1. Recollection on derived algebraic geometry. In this part we recall the terminology on derived schemes.

We start with the terminology on affine derived schemes, some of which are already recalled in Notations 1.4.1 and 1.4.2.

(1) We denote by dAff the $\infty$-category of affine derived schemes over $\mathbb{k}$. It is the opposite of the $\infty$ category $\mathbf{d g u C o m}^{\leq 0}$ of cdgas over $\mathbb{k}$ concentrated in the non-positive cohomological degrees . For a cdga $R \in \mathbf{d g u C o m} \leq 0$, we denote by $\operatorname{Spec}(R) \in \mathbf{d A f f}$ the corresponding affine derived scheme.

(2) For $R=\left(R, d_{R}\right) \in \mathbf{d g u C o m}^{\leq 0}$ and $n \in \mathbb{N}$, we denote by

$$
\pi_{n}(R):=H^{-n}\left(R, d_{R}\right)
$$

its $(-n)$-th cohomology (Definition 1.1.7). Actually it coincides with the $n$-th homotopy group of the differential graded nerve of $\left(R, d_{R}\right)$. See $[L u 2, \S 1.3 .1]$ for the detail. In particular, we have the functor

$$
\pi_{0}: \mathbf{d g u C o m}^{\leq 0} \longrightarrow \text { Comu }
$$

of $\infty$-categories. where Comu denotes the $(\infty$-) category of commutative $\mathbb{k}$-algebras. Recall here that a functor of $\infty$-category means a morphism of simplicial sets [Lu1, §1.2.7], The functor $\pi_{0}$ is called the truncation (functor).

(3) We also have the inclusion functor $\iota:$ Comu $\rightarrow \mathbf{d g u C o m}^{\leq 0}$ whose definition is an obvious one. These two functors form an adjunction

$$
\pi_{0}: \operatorname{dguCom}^{\leq 0} \rightleftarrows \text { Comu : } \iota
$$

of functors of $\infty$-categories. See [Lu1, §5.2] for the detail of adjunctions of functors between $\infty$ categories. In particular, for $A \in \mathbf{d g u C o m}^{\leq 0}$ and $B \in$ Comu, we have an isomorphism

$$
\operatorname{Map}_{\text {Comu }}\left(\pi_{0}(A), B\right) \stackrel{\sim}{\longrightarrow} \operatorname{Map}_{\text {dguCom }} \leq 0(A, \iota(B))
$$

in the homotopy category $\mathcal{H}$ of spaces $(\S 0.3)$. Taking $\pi_{0}$ of the mapping spaces, we recover the ordinary adjunction property $\operatorname{Hom}_{\mathrm{Comu}}\left(\pi_{0}(A), B\right) \stackrel{\sim}{\rightarrow} \operatorname{Hom}_{\mathbf{d g u C o m} \leq 0}(A, \iota(B))$.

(4) We will use Zariski open immersions, étale, smooth, flat and locally finitely presented morphisms in $\mathbf{d g u C o m}^{\leq 0}$ or dAff. See [TVe, $\left.§ 1.2 .6\right]$ for the precise definitions.

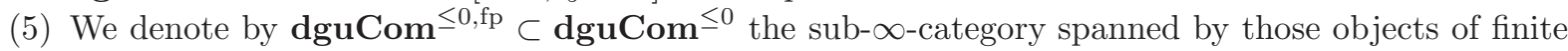
presentation over $\mathbb{k}$ in the sense of [TVe, Definition 1.2.3.1]. As noted in [TVe, §2.2.1], the truncation functor $\pi_{0}$ gives

$$
\operatorname{dguCom}^{\leq 0, \mathrm{fp}} \longrightarrow \text { Comu }^{\mathrm{ft}},
$$

where Comu $^{\mathrm{ft}} \subset$ Comu denotes the subcategory of finite type objects. We also denote by $\mathbf{d A f f}{ }^{\mathrm{fp}}:=$ $\left(\mathbf{d g u} \mathbf{C o m}^{\leq 0, f \mathrm{p}}\right)^{\text {op }}$ and call its object an affine derived scheme of finite presentation over $\mathbb{k}$.

(6) The fiber product in dAff is denoted by $X \times_{Y}^{\mathbb{L}} Z$. It corresponds to the derived tensor product $A \otimes_{B}^{\mathbb{L}} C$ in $\mathbf{d g u C o m}^{\leq 0}$, and is represented by the two-sided bar complex (Definition 2.1.8). 
Next we turn to the terminology on derived stacks and derived schemes. Recall that we denote by $\mathcal{S}$ the $\infty$-category of spaces $(\S 0.3)$.

(6) A $D^{-}$-stack over $\mathbb{k}$ in the sense of [TVe, Chap. 2.2] is called a derived stack. Thus, it is a functor

$$
F: \mathbf{d A f f}^{\text {op }} \longrightarrow \mathcal{S}
$$

of $\infty$-categories satisfying the sheaf condition for the étale $\infty$-topos ét on dAff. We can construct an $\infty$-category of derived stacks by [TVe, $\S 1.3 .2$ ], and we denote it by dSt.

(7) An affine derived scheme $\operatorname{Spec}(A)$ with $A \in \mathbf{d A f f}$ defines a derived stack by the Yoneda embedding

$$
\underline{\operatorname{Map}}_{\mathbf{d A f f}}(-, \operatorname{Spec}(A)): \mathbf{d A f f}{ }^{\text {op }} \longrightarrow \mathcal{S} .
$$

Here, for an $\infty$-category $\mathcal{C}$, we denoted by $\underline{\operatorname{Map}}_{\mathcal{C}}(-,-)$ the Kan simplicial set such that its homotopy type is $\operatorname{Map}_{\mathcal{C}}(-,-)$. The existence of such a simplicial set is shown in [Lu1, §1.2.2], where it is called the space of right morphisms. A derived stack which is equivalent to the one of the form $\underline{\operatorname{Map}}_{\mathrm{dAff}}(-, \operatorname{Spec}(A))$ is called a representable derived stack [TVe, §1.3.2]. We identify an affine derived scheme and a representable derived stack.

(8) A geometric $D^{-}$-stack over $\mathbb{k}$ in the sense of [TVe, $\left.\$ 2.2 .3\right]$ will be called a geometric derived stack. For $m \in \mathbb{Z}_{\geq-1}$, one defines an $n$-geometric derived stack inductively on $m$. An (-1)-geometric derived stack is defined to be a representable derived stack, and the inductive step defines an $m$-geometric derived stack to be a derived stack having an atlas of $(m-1)$-geometric derived stacks with respect to the smooth morphism in dAff.

(9) For $n \in \mathbb{N}$, a derived stack $F$ is $n$-truncated if $\pi_{i}(F(T), s)=0$ for any $i \in \mathbb{Z}_{>n}$, any $T \in \mathbf{d A f f}$ and any $s \in \pi_{0}(F(T)$ ). A derived scheme (over $\mathbb{k}$ ) is defined to be an $n$-truncated $m$-geometric derived stack $X$ with some $m$ and $n$ such that there is an $n$-atlas $\sqcup_{i} U_{i} \rightarrow X$ of affine derived schemes $U_{i}$ 's and each $U_{i} \rightarrow X$ is a monomorphism of stacks [TVe, Definition 2.1.1.4]. By [TVe, Remark 2.1.1.5 (1)], a derived scheme is automatically 1-geometric. Equivalently, a derived stack is a pair $\left(X, \mathcal{O}_{X}\right)$ of a topological space $X$ and a sheaf $\mathcal{O}_{X}$ valued in $\mathbf{d g u C o m}^{\leq 0}$ such that the truncation $\left(X, \pi_{0}\left(\mathcal{O}_{X}\right)\right)$ is an ordinary scheme and the sheaf $\pi_{i}\left(\mathcal{O}_{X}\right)$ is a quasi-coherent sheaf of $\pi_{0}\left(\mathcal{O}_{X}\right)$-modules. We denote by $\mathbf{d} \mathbf{S c h} \subset \mathbf{d} \mathbf{S t}$ the sub- $\infty$-category spanned by derived schemes.

(10) We denote by $\mathbf{d S t}^{\mathrm{fp}} \subset \mathbf{d} \mathbf{S t}$ and $\mathbf{d} \mathbf{S} \mathbf{c h}{ }^{\mathrm{fp}} \subset \mathbf{d} \mathbf{S c h}$ the sub- $\infty$-categories spanned by those objects of categorically locally finite presentation over $\mathbb{k}$ in the sense of [TVe, Definition 1.3.6.4].

3.2.2. Jet and arc spaces for derived schemes. We continue to use the notations given in the lase §3.2.1. In particular, we denote by $\mathbf{d S c h}$ the $\infty$-category of derived schemes over $\mathbb{k}$.

We have the following derived analogue of the functor $J_{n}$ in Fact 3.1.1.

Proposition 3.2.1. For any $n \in \mathbb{N}$, there is a functor $J_{n}: \mathbf{d} \mathbf{S c h} \rightarrow \mathbf{d S c h}$ of $\infty$-categories such that we have an functorial isomorphism

$$
\operatorname{Map}_{\mathbf{d S c h}}\left(S, J_{n}(X)\right) \simeq \operatorname{Map}_{\mathbf{d S c h}}\left(S \times{ }_{\operatorname{Spec}(\mathbb{k})}^{\mathbb{L}} \operatorname{Spec}\left(\mathbb{k}[t] /\left(t^{n+1}\right)\right), X\right)
$$

in the homotopy category $\mathcal{H}$ of spaces. We call $J_{n}(X)$ the $n$-th jet space of $X$.

Our proof basically follows the non-derived case of [BLR, $\S 7.6$, Theorem 4], but we add some modification to work correctly in derived algebraic geometry.

For $X \in \mathbf{d S c h}$, we define a functor $\underline{J_{n}}(X): \mathbf{d S c h}{ }^{\text {op }} \rightarrow \mathcal{S}$ of $\infty$-categories by

$$
\underline{J_{n}}(X)(S):=\underline{\operatorname{Map}}_{\mathrm{dSch}}\left(S \times{ }_{\operatorname{Spec}(\mathbb{k})}^{\mathbb{L}} \operatorname{Spec}\left(\mathbb{k}[t] /\left(t^{n+1}\right)\right), X\right) .
$$

We first show:

Lemma 3.2.2. The functor $\underline{J_{n}}(X)$ is a derived stack.

Proof. For any derived stacks $G, H \in \mathbf{d S t}$, we have the internal derived stack $\mathbb{R}_{\text {ét }} \underline{\operatorname{Hom}}_{\mathrm{dSt}}(G, H) \in \mathbf{d S t}$ satisfying the adjunction isomorphisms $\operatorname{Map}_{\mathbf{d S t}}\left(F, \mathbb{R}_{\text {ét }} \underline{\operatorname{Hom}}(G, H)\right) \simeq \operatorname{Map}_{\mathbf{d S t}}\left(F \times_{\operatorname{Spec}(\mathbb{k})}^{\mathbb{L}} G, H\right)$ for any $F \in$ dSt. See [TVe, $\S 1.4 .1]$ for the detail. Taking $G:=\operatorname{Spec}\left(\mathbb{k}[t] /\left(t^{n+1}\right)\right)$ and $H=X$, we have the consequence.

Thus we want to show that this derived stack is (represented by) a derived scheme $J_{n}(X)$. The case $n=0$ is trivial: $J_{0}(X)=X$.

Note that for a morphism $u: X \rightarrow Y$ in $\mathbf{d S c h}$, we have the induced morphism of functors:

$$
\underline{J_{n}}(u): \underline{J_{n}}(X) \longrightarrow \underline{J_{n}}(Y) .
$$


In particular, for $m, n \in \mathbb{N}$ with $m \geq n$, the truncation morphism $\mathbb{k}[t] /\left(t^{m+1}\right) \rightarrow \mathbb{k}[t] /\left(t^{n+1}\right)$ induces a morphism $\pi_{m, n}: \underline{J_{m}}(X) \rightarrow \underline{J_{n}}(X)$ of functors. Then we can check the following statement by a set-theoretic argument (see [EM09, Lemma 2.3] for the detail).

Lemma 3.2.3. Let $u: U \hookrightarrow X$ be a monomorphism in dSch from an affine derived scheme $U$ to a derived scheme $X$. If a derived scheme $J_{n}(X)$ representing the functor $J_{n}(X)$ exists, then the representing derived scheme $J_{n}(U)$ exists and we have $J_{n}(U) \simeq \pi_{n, 0}^{-1}(U)$, where $\pi_{n, 0}: J_{n}(X) \rightarrow J_{0}(X)=X$ is the induced morphism from the truncation morphism $\mathbb{k}[t] /\left(t^{n+1}\right) \rightarrow \mathbb{k}$.

Next we show the existence of the representing object in the affine case. Recall that we denote by $\mathbf{d} \mathbf{A} \mathrm{ff}^{\mathrm{fp}}$ the $\infty$-category of affine derived schemes of finite presentations over $\mathbb{k}(\S 3.2 .1(5))$.

Lemma 3.2.4. Let $n \in \mathbb{N}$. For any $R \in \mathbf{d g u C o m}^{\leq 0}$, the functor

$$
\underline{J_{n}}(R): \operatorname{dguCom} \leq 0 \longrightarrow \mathcal{S}, \quad \underline{J}_{n}(R)(A):=\underline{\operatorname{Map}}_{\operatorname{dguCom} \leq 0}\left(R, A \otimes_{\mathbb{k}}^{\mathbb{L}} \mathbb{k}[t] /\left(t^{n+1}\right)\right)
$$

of $\infty$-categories is represented by $J_{n}(R) \in \mathbf{d g u C o m}^{\leq 0}$. Moreover the correspondence $R \mapsto J_{n}(R)$ determines a functor $J_{n}: \operatorname{dguCom}^{\leq 0} \rightarrow \mathbf{d g u C o m}^{\leq 0}$ of $\infty$-categories.

Proof. We may replace an $R \in \mathbf{d g u} \mathbf{C o m}^{\leq 0}$ by a free resolution, i.e., a cdga $\widetilde{R} \in \mathbf{d g u C o m}{ }^{\leq 0}$ such that

- the underlying graded algebra is a free algebra over $k$, and

- it is a K-flat complex quasi-isomorphic to $R$.

We cite from [SP, 0BZ6, 0BZ67 Lemma] an explicit construction of such a resolution:

(i) Take a set of homogeneous elements $r_{s} \in \operatorname{Ker}\left(d_{R}: R \rightarrow R\right)\left(s \in S_{0}\right)$ such that the classes $\overline{r_{s}} \in$ $H\left(R, d_{R}\right)$ generate the linear space $H\left(R, d_{R}\right)$. We define a cdga

$$
R_{0}=\mathbb{k}\left[x^{0, s} \mid s \in S_{0}\right]
$$

to be the free polynomial graded algebra generated by the letters $\left\{x^{0, s} \mid s \in S_{0}\right\}$ equipped with the grading $\left|x^{0, s}\right|:=\left|r_{0, s}\right|$ and trivial differential. We have a morphism $f_{0}: R_{0} \rightarrow R$ of cdgas given by $x^{0, s} \mapsto r_{0, s}$. It is obvious that $R_{0}$ is a free commutative algebra over $\mathbb{k}$ and a $\mathrm{K}$-flat complex, and that the induced morphism $H\left(f_{0}\right): H\left(R_{0}\right) \rightarrow H(R)$ is a surjection.

(ii) Assume that we have constructed a sequence

$$
R_{0} \rightarrow R_{1} \rightarrow \cdots \rightarrow R_{m-1} \stackrel{i_{m-1}}{\longrightarrow} R_{m} \stackrel{f_{m}}{\longrightarrow} R
$$

of cdgas. Take a set of homogeneous elements $r_{m, s} \in R_{m}\left(s \in S_{m}\right)$ such that the classes $\overline{r_{m, s}} \in$ $H\left(R_{m}, d_{R_{m}}\right)$ span the linear space $\operatorname{Ker}\left(H\left(f_{m}\right): H\left(R_{m}, d_{R_{m}}\right) \rightarrow H\left(R, d_{R}\right)\right)$. We define a cdga

$$
R_{m+1}=R_{m}\left[x^{m, s} \mid s \in S_{m}\right]
$$

to be the free polynomial graded algebra generated by the letters $\left\{x^{m, s} \mid s \in S_{m}\right\}$ over $R_{m}$ equipped with the grading $\left|x^{m, s}\right|:=\left|r_{m, s}\right|-1$ and the differential $d_{R_{m+1}}\left(x^{m, s}\right):=r_{m, s}$ and $\left.d_{R_{m+1}}\right|_{R_{m}}:=d_{R_{m}}$. We have a natural embedding $i_{m}: R_{m} \hookrightarrow R_{m+1}$ of cdgas, and also have a morphism $f_{m+1}: R_{m+1} \rightarrow$ $R$ of cdgas given by $f_{m+1}\left(x^{m, s}\right):=f_{m}\left(r_{m, s}\right)$ and $\left.f_{m+1}\right|_{R_{m}}:=f_{m}$. By induction, we obviously have that $R_{m+1}$ is a free commutative algebra over $\mathbb{k}$ and the induced morphism $H\left(f_{m+1}\right): H\left(R_{m+1}\right) \rightarrow$ $H(R)$ is a surjection. We can also check that $R_{m+1}$ is K-flat.

(iii) Finally we define

$$
\widetilde{R}:=\operatorname{colim}_{m} R_{m},
$$

where the colimit is taken in the $\infty$-category $\operatorname{dguCom}^{\leq 0}$. We also have a morphism $f: \widetilde{R} \rightarrow R$ of cdgas. We can check that $\widetilde{R}$ is a free graded algebra over $\mathbb{k}$ and a K-flat complex, and that the induced morphism $H(f)$ is an isomorphism.

Note that this construction respects quasi-isomorphisms: If we are given a quasi-isomorphism $A \rightarrow B$ of cdgas, then the construction induces a quasi-isomorphism $\widetilde{A} \rightarrow \widetilde{B}$.

Now we will define $J_{n}(R)$ by constructing $J_{n}\left(R_{m}\right)$ 's inductively on $m$ and setting

$$
J_{n}(R)=J_{n}(\widetilde{R}):=\operatorname{colim}_{m} J_{n}\left(R_{m}\right) .
$$

In the case $m=0$, we have $R_{0}=\mathbb{k}\left[x^{0, s} \mid s \in S_{0}\right]$. Then, similarly as in Remark 3.1.4 (1), the desired cdga is given by the polynomial graded algebra

$$
J_{n}\left(R_{0}\right)=\mathbb{k}\left[x_{(-j-1)}^{0, s} \mid s \in S_{0}, j=0, \ldots, n\right]
$$


with $\left|x_{(-j-1)}^{0, s}\right|:=\left|x^{0, s}\right|$ and trivial differential. Recalling Fact 3.1.6, we equip with $J_{n}\left(R_{0}\right)$ with the 0derivation $T_{0}$ given by $T_{0}\left(x_{(-j)}^{0, s}\right)=j x_{(-j-1)}^{0, s}$. The pair $\left(J_{n}\left(R_{0}\right), T_{0}\right)$ represents the functor $\underline{J_{0}}\left(R_{0}\right)$.

Assume that we have constructed a pair $\left(J_{n}\left(R_{d}\right), T_{d}\right)$ representing the functor $\underline{J_{d}}\left(R_{d}\right)$ for $d \leq m$. Note that as a commutative graded algebra $R_{m+1}$ is isomorphic to $R_{m}\left[x^{m, s}, y^{m, s} \mid s \in S_{m}\right] /\left(y^{m, s}-r_{m, s} \mid s \in S_{m}\right)$, with the grading $\left|y^{m, s}\right|=\left|r_{m, s}\right|$. Then, similarly as in Remark 3.1.4 (2), we define the cdga $J_{n}\left(R_{m}\right)$ by

$$
J_{n}\left(R_{m+1}\right):=J_{n}\left(R_{m}\right)\left[x_{(-j-1)}^{m, s}, y_{(-j-1)}^{m, s} \mid s \in S_{m}, j=0, \ldots, n\right] /\left(T_{m+1}^{k} g_{m, s} \mid k=0, \ldots, n, s \in S_{m}\right),
$$

where $g_{m, s}:=y_{(-1)}^{m, s}-r_{m, s}\left(x_{(-1)}^{m, t}\right) \in R_{m}\left[x_{(-1)}^{m, t}, y_{(-1)}^{m, s} \mid t \in S\right]$ with $r_{m, s}=r_{m, s}\left(x^{m, t}\right)$ regarded as a polynomial of $x^{m, t}$ s, and $T_{m+1}$ is a 0 -derivation defined by

$$
T_{m+1} x_{(-j)}^{m, s}:=j x_{(-j-1)}^{m, s}, \quad T_{m+1} y_{(-j)}^{m, s}:=j y_{(-j-1)}^{m, s},\left.\quad T_{m+1}\right|_{J_{n}\left(R_{m}\right)}:=T_{m} .
$$

The cohomological grading is given by $\left|x_{(-j)}^{m, s}\right|:=\left|x^{m, s}\right|=\left|r^{m, s}\right|-1$ and $\left|y_{(-j)}^{m, s}\right|:=\left|y^{m, s}\right|=\left|r^{m, s}\right|$, and the differential $d_{m+1}$ is given by $d_{m+1} T_{m+1}=T_{m+1} d_{m+1}, d_{m+1}\left(x_{(-1)}^{m, s}\right):=y_{(-1)}^{m, s}, d_{m+1}\left(y_{(-1)}^{m, s}\right):=r_{m, s}\left(x_{(-1)}^{m, t}\right)$. Then the pair $\left(J_{n}\left(R_{m+1}\right), T_{m+1}\right)$ represents the functor $J_{n}\left(R_{m+1}\right)$.

The injection $i_{m}: R_{m} \hookrightarrow R_{m+1}$ induces a cofibration $\overline{J_{n}}\left(i_{m}\right): J_{n}\left(R_{m}\right) \rightarrow J_{n}\left(R_{m+1}\right)$ in $\mathbf{d g u C o m} \leq 0$ which (homotopically) commutes with the 0 -derivations $T_{m}$ and $T_{m+1}$, and we can take the colimit $\left(J_{n}(\widetilde{R}), T\right):=$ $\operatorname{colim}_{m}\left(J_{n}\left(R_{m}\right), T_{m}\right)$. The pair $\left(J_{n}(\widetilde{R}), T\right)$ represents the functor $\underline{J_{n}}(R)$ by construction.

By the construction, we immediately have

Corollary. In Lemma 3.2.4, if $R \in \mathbf{d g u C o m}^{\leq 0, \text { fp }}$, then we have $J_{n}(R) \in \mathbf{d g} \mathbf{u} \mathbf{C o m} \leq 0$,fp . We also have a functor $J_{n}: \mathbf{d g u C o m} \leq 0, \mathrm{fp} \rightarrow \mathbf{d g u C o m}^{\leq 0, \mathrm{fp}}$ of $\infty$-categories.

Let us give the proof of Proposition 3.2.1.

Proof of Propoisition 3.2.1. By the definition of a derived scheme $X(\S 3.2 .1,(9))$, we have an atlas $\sqcup_{i} U_{i} \rightarrow X$ of $X$ consisting of affine derived schemes $U_{i}$. By Lemma 3.2.4, we have the representing derived scheme $J_{n}\left(U_{i}\right)$. These satisfy the gluing condition by Lemma 3.2.3. Thus we have a derived stack representing the functor $J_{n}(X)$. the consequence.

Remark. Let us comment a more natural point of view in the context of derived algebraic geometry. In Lemma 3.2.2 we recalled the internal derived stack $\mathbb{R}_{\text {ét }} \underline{\operatorname{Hom}}_{\mathbf{d S t}}(G, H) \in \mathbf{d S t}$ for derived stacks $G$ and $H$. If $G$ is a scheme and $H$ is an $m$-geometric derived stack for some $m \in \mathbb{Z}_{\geq-1}$, then it is denoted by $\operatorname{Map}(G, H)$ and called the mapping derived stack. By [TVe, Theorem 2.2.6.11], the mapping derived $\operatorname{stack} \operatorname{Map}(G, H)$ is also $m$-geometric under some conditions, which can be checked for our case $G=D_{n}:=\operatorname{Spec}\left(k[t] /\left(t^{n+1}\right)\right)$ and $H=X \in \mathbf{d S c h}$ with $m=1$. Thus the $n$-th jet scheme is nothing but the mapping derived stack from the $n$-fattened infinitesimal disk $D_{n}$ :

$$
J_{n}(X) \simeq \operatorname{Map}\left(D_{n}, X\right) .
$$

By the construction, the $n$-th jet spaces of derived schemes enjoy similar properties as those of non-derived schemes in Fact 3.1.3.

Lemma. Let $X$ be a derived scheme over $\mathbb{k}$ and $n \in \mathbb{N}$.

(1) We have $J_{0}(X) \simeq X$.

(2) For $m \in \mathbb{N}$ with $m \geq n$, the truncation morphism $\mathbb{k}[t] /\left(t^{m+1}\right) \rightarrow \mathbb{k}[t] /\left(t^{n+1}\right)$ induces a morphism $\pi_{m, n}: J_{m}(X) \rightarrow J_{n}(X)$ in dSch. The morphisms $\left\{\pi_{m, n}: J_{m}(X) \rightarrow J_{n}(X) \mid m, n \in \mathbb{N}, m \geq n\right\}$ form an inverse system over the direct set $(\mathbb{N}, \geq)$.

(3) For $R \in \mathbf{d g u C o m}^{\leq 0 \text {,fp }}$, we have $J_{n}(R) \in \mathbf{d g u C o m}^{\leq 0 \text {,fp }}$.

(4) The (homotopy) fiber of the morphism $\pi_{n, 0}: J_{n}(X) \rightarrow J_{0}(X)=X$ is equivalent to an affine derived scheme.

The last property guarantee the existence of the arc space of derived schemes.

Proposition. For $X \in \mathbf{d S c h}$, the limit of the inverse system $\left\{\pi_{m, n}: J_{m}(X) \rightarrow J_{n}(X) \mid m, n \in \mathbb{N}, m \geq n\right\}$ exists in $\mathbf{d S c h}$. We denote it by $J_{\infty}(X)$, and call it the arc space or the $\infty$-jet space of $X$.

In the sections below, we only deal with cdgas $R$ (but not necessarily concentrated in non-positive degrees). For later reference, we set:

Definition 3.2.5. For a cdga $R$, we call the cdga $J_{\infty}(R)$ with 0 -derivation the arc space of $R$. 


\section{Li filtration AND VERTEX POISSON ALGEBRAS IN DERIVED SETting}

Recall that given an affine Poisson scheme, the coordinate ring of its jet scheme has a structure of vertex Poisson algebra [A15]. In this section we give a dg analogue of this statement. We will work over a field $\mathbb{k}$ of characteristics 0 .

4.1. Dg vertex algebras. In this subsection we recall basics of vertex algebras and define dg vertex algebras. In order to make the text consistent with the literature of vertex algebras, we begin with the preliminary on super objects.

4.1.1. Super convention. In this part we collect the convention on super objects. We express $\mathbb{Z} / 2 \mathbb{Z}=\{\overline{0}, \overline{1}\}$.

Definition 4.1.1. (1) A linear superspace is a $\mathbb{Z} / 2 \mathbb{Z}$-graded linear space.

(2) The $\mathbb{Z} / 2 \mathbb{Z}$-grading of a linear superspace $V$ is called the parity, and the grade decomposition of $V$ is denoted by $V=V_{\overline{0}} \bigoplus V_{\overline{1}}$. A homogeneous element $v$ of $V$ is also called an element of pure parity, and we denote by $p(v)$ its parity. An element of $V_{\overline{0}}$ is called even, and an element of $V_{\overline{1}}$ is called odd.

(3) Let $V$ and $W$ be linear superspaces. A linear map $f: V \rightarrow W$ is called even if $f\left(V_{\bar{i}}\right) \subset W_{\bar{i}}$ for $i=0,1$, and called odd if $f\left(V_{\bar{i}}\right) \subset W_{\overline{i+1}}$ for $i=0,1$. We denote by

$$
\operatorname{Hom}_{\mathbb{k}}(V, W)=\operatorname{Hom}_{\mathbb{k}}(V, W)_{\overline{0}} \oplus \operatorname{Hom}_{\mathbb{k}}(V, W)_{\overline{1}}
$$

the corresponding linear superspace structure on $\operatorname{Hom}_{\mathbb{k}}(V, W)$.

(4) The category of linear superspaces and even linear maps will be denoted by sVec. Thus we have $\operatorname{Hom}_{\mathbf{s} \text { ec }}(V, W)=\operatorname{Hom}_{\mathbb{k}}(V, W)_{\overline{0}}$.

Definition 4.1.2. Let $V$ and $W$ be linear superspaces.

(1) The tensor product $V \otimes W \in \mathrm{sVec}$ of $V$ and $W$ is defined to be the linear superspace whose underlying linear space is $V \otimes_{\mathbb{k}} W \in \mathrm{Vec}$ and the parity decomposition is given by

$$
(V \otimes W)_{\overline{0}}:=V_{\overline{0}} \otimes_{\mathbb{k}} W_{\overline{0}} \oplus V_{\overline{1}} \otimes_{\mathbb{k}} W_{\overline{1}}, \quad(V \otimes W)_{\overline{1}}:=V_{\overline{0}} \otimes_{\mathbb{k}} W_{\overline{1}} \oplus V_{\overline{1}} \otimes_{\mathbb{k}} W_{\overline{0}} .
$$

(2) The braiding (or the commutativity) isomorphism on the tensor product $V \otimes W$ is an isomorphism in $\mathrm{s} V e c$ given by

$$
V \otimes W \stackrel{\sim}{\longrightarrow} W \otimes V, \quad v \otimes w \longmapsto(-1)^{p(v) p(w)} w \otimes v
$$

for elements $v \in V, w \in W$ of pure parity.

These define a symmetric monoidal structure on $\mathrm{sVec}$, which is denoted by $\mathrm{sVec}^{\otimes}$.

The tensor product and the braiding isomorphism gives sVec a structure of symmetric monoidal category. Thus we have notions of associative algebras, commutative algebras and Lie superalgebras. In particular, for a linear superspace $V=V_{\overline{0}} \oplus V_{\overline{1}}$, we have the endomorphism superalgebra $\operatorname{End}_{\mathbb{k}}(V)$ and the commutator

$$
[f, g]:=f g-(-1)^{p(f) p(g)} g f
$$

for $f, g \in \operatorname{End}_{\mathbb{k}}(V)$ of pure parity $p(f)$ and $p(g)$ respectively. We will repeatedly use this commutator.

Recall the notion of a differential algebra (Definition 3.1.7). For later use, let us give a super analogue.

Definition 4.1.3. Let $R$ be a superalgebra.

(1) For $\varepsilon \in \mathbb{Z} / 2 \mathbb{Z}$, a derivation $d$ of parity $\varepsilon$ on $R$ is a linear endomorphism on $R$ of parity $\varepsilon$ such that for any $r \in R$ we have $[d, r]=d r$ in the endomorphism superalgebra $\operatorname{End}(R)$, where in the left hand side we regard $r \in \operatorname{End}(R)$ as a multiplication operator.

(2) We denote by $\operatorname{Der}(R)_{\varepsilon}$ the linear space of derivations of parity $\varepsilon$ on $R$.

(3) A superalgebra equipped with a derivation is called a differential superalgebra.

Finally we remark a construction of super objects from graded objects. See [BD, 1.1.16] for a more systematic "dg super" conventions.

Notation 4.1.4. (1) For a complex $V=\left(V^{\bullet}, d\right)$, we have a linear superspace whose even part is $V^{\text {even }}:=\bigoplus_{n \in \mathbb{Z}} V^{2 n}$ and whose odd part is $V^{\text {odd }}:=\bigoplus_{n \in \mathbb{Z}} V^{2 n+1}$. We denote it by $V^{\text {even }} \oplus V^{\text {odd }}$.

(2) The correspondence $V \mapsto V^{\text {even }} \oplus V^{\text {odd }}$ defines a monoidal functor $\operatorname{dgVec}^{\otimes} \rightarrow \mathrm{sVec}^{\otimes}$, and it induces similar functors for algebraic structures. We call those objects lying in the essential image the associated super objects of given dg objects.

For example, for a graded Lie algebra $L=L^{\bullet}$, we have the associated Lie superalgebra $L^{\text {even }} \oplus L^{\text {odd }}$. 
4.1.2. Vertex superalgebras. For the definiteness, we begin with the recollection of vertex superalgebras. See [Ka] and [FBZ] for the detail.

Notation. (1) We denote by $\mathbb{k}[[z]]$ the linear space of formal series, and by $\mathbb{k}((z))$ the linear space of formal Laurent series. Thus we have a decomposition $\mathbb{k}((z))=\mathbb{k}[[z]] \oplus z^{-1} \mathbb{k}\left[z^{-1}\right]$.

(2) For a linear space $V$, we denote $V[[z]]:=V \otimes_{\mathbb{k}} \mathbb{k}[[z]] . V((z)):=V \otimes_{\mathbb{k}} \mathbb{k}((z)), z V[z]:=V \otimes_{\mathbb{k}} z \mathbb{k}[z]$ and so on.

Definition 4.1.5. A vertex superalgebra (vsa for short) is a data $(V,|0\rangle, T, Y)$ consisting of

- a linear superspace $V$,

- an even element $|0\rangle \in V_{\overline{0}}$ called the vacuum,

- an even endomorphism $T \in \operatorname{End}(V)_{\overline{0}}$ called the translation, and

- a linear map

$$
V \otimes V \longrightarrow V((z)), \quad a \otimes b \longmapsto Y(a, z) b=\sum_{n}\left(a_{(n)} b\right) z^{-n-1}
$$

such that we have $a_{(n)} \in \operatorname{End}(V)_{\varepsilon}$ for $a \in V_{\varepsilon}$ of pure parity and for any $n \in \mathbb{Z}$. The operation $Y$ is called the state-field correspondence.

These should satisfy the following standard axioms.

(i) (Vacuum axiom) $Y(|0\rangle, z)=\operatorname{id}_{V}$ and $Y(a, z)|0\rangle \in a+z V[[z]]$ for any $a \in V$.

(ii) (Translation axiom) $T|0\rangle=0$ and $[T, Y(a, z)]=\partial_{z} Y(a, z)$ for any $a \in V$.

(iii) (Locality axiom) $Y(a, z)$ 's are mutually local, i.e., for any homogeneous $a, b \in V$, there exists $N \in \mathbb{N}$ such that the following equation holds in $(\operatorname{End}(V))\left[\left[z^{ \pm 1}, w^{ \pm 1}\right]\right]$.

$$
(z-w)^{N} Y(a, z) Y(b, w)=(-1)^{p(a) p(b)}(z-w)^{N} Y(b, w) Y(a, z) .
$$

We often denote it simply by $V$.

An even vertex superalgebra, i.e., $V=V_{\overline{0}}$, is called a vertex algebra (va for short).

Several remarks are in order.

Remark 4.1.6. (1) The locality axiom implies the following relations.

$$
\begin{aligned}
& {\left[a_{(m)}, b_{(n)}\right]=\sum_{l \in \mathbb{N}}\left(\begin{array}{c}
m \\
l
\end{array}\right)\left(a_{(l)} b\right)_{(m+n-l)},} \\
& \left(a_{(m)} b\right)_{(n)} c=\sum_{l \in \mathbb{N}}(-1)^{l}\left(\begin{array}{c}
m \\
l
\end{array}\right)\left(_{(m-l)}\left(b_{(n+l)} c\right)-(-1)^{m+p(a) p(b)} b_{(m+n-l)}\left(a_{(l)} c\right)\right),
\end{aligned}
$$

where $a, b, c \in V$ and $a, b$ are of pure parity. We also have the skew-symmetry

$$
Y(a, z) b=(-1)^{p(a) p(b)} e^{z T} Y(b,-z) a
$$

for homogeneous $a, b \in V$. See [FBZ, Chap. 3] and [Ka, $\S 44.2,4.6,4.8]$ for the detail.

(2) The translation $T$ is completely determined by the operation $Y$ as $T a=a_{(-2)}|0\rangle$.

(3) The correspondence $a \mapsto a_{(-1)}$ is injective [FBZ, 1.3.2, Remarks 4].

Let us also recall:

Definition. Let $V$ and $W$ be vertex superalgebras.

(1) A morphism $V \rightarrow W$ of vsas is an even linear map $\varphi: V \rightarrow W$ such that $\varphi\left(a_{(n)} b\right)=\varphi(a)_{(n)} \varphi(b)$ for any $a, b \in V$ and $n \in \mathbb{Z}$.

(2) The tensor product $V \otimes W$ of vsas is given by $\left(V \otimes W,|0\rangle_{V} \otimes|0\rangle_{W}, T_{V} \otimes \mathrm{id}_{W}+\mathrm{id}_{V} \otimes T_{W}, Y_{V \otimes W}\right)$, where the first item denotes the tensor product as linear superspaces (Definition $4.1 .2(1)$ ) and $Y_{V \otimes W}(a \otimes b, z):=Y_{V}(a, z) \otimes Y_{W}(b, z)$. Thus we have $(a \otimes b)_{(n)}=\sum_{m \in \mathbb{Z}} a_{(m)} \otimes b_{(n-m-1)}$ using the tensor product $(f \otimes g)(c \otimes d)=(-1)^{p(g) p(c)} f(c) \otimes g(d)$ of linear operators $f$ and $g$ coming from the braiding isomorphism (Definition 4.1.2 (2)).

Note that the tensor product $\otimes$ gives a unital symmetric monoidal structure on the category of vsas, where the unit is the trivial vertex algebra $\mathbb{k}$.

We also have the standard notion of vertex super subalgebras and those of ideals and quotients of vsas. See $[\mathrm{Ka}, \S 4.3]$ for the detail.

Finally we give some terminology for modules over vsas. There are several different definitions, and we cite a version from $[\mathrm{A} 12, \S 2.1]$. 
Definition. Let $V$ be a vertex superalgebra. A $V$-module is a linear superspace $M$ equipped with a linear map

$$
V \otimes M \longrightarrow M((z)), \quad a \otimes m \longmapsto Y^{M}(a, z) m=\sum_{k \in \mathbb{Z}} a_{(k)}^{M} m z^{-k-1}
$$

where for $a \in V_{\varepsilon}$ we have $a_{(k)}^{M} \in \operatorname{End}(M)_{\varepsilon}$, which should satisfy the following conditions.

(i) $Y^{M}(|0\rangle, z)=\operatorname{id}_{M}$,

(ii) For elements $a, b \in V$ of pure parity and any $j, k, l \in \mathbb{Z}$, we have

$$
\sum_{n \in \mathbb{N}}\left(\begin{array}{l}
k \\
n
\end{array}\right)\left(a_{(l+n)} b\right)_{k+j-n}^{M}=\sum_{n \in \mathbb{N}}(-1)^{n}\left(\begin{array}{l}
l \\
n
\end{array}\right)\left(a_{(k+l-n)}^{M} b_{(j+n)}^{M}-(-1)^{l+p(a) p(b)} b_{(l+j-n)}^{M} a_{(k+n)}^{M}\right) .
$$

A morphism of $V$-modules is naturally defined. We denote by $V$-VMod the category of $V$-modules.

Example 4.1.7. Let $\varphi: V \rightarrow W$ be a morphism of vsas. Then $W$ is naturally a $V$-module. In fact, denoting the state-field correspondence of $W$ as a vsa by $\alpha \otimes \beta \mapsto Y_{W}(\alpha, z) \beta$, we have the $V$-module structure $V \otimes W \rightarrow W((z)), Y^{W}(a, z) \beta:=Y_{W}(\varphi(a), z) \beta$ for $a \otimes \beta \in V \otimes W$.

Let us recall an equivalent description of modules over vertex superalgebras.:

Fact 4.1.8 $([\mathrm{FBZ}, 5.1 .6$. Theorem $])$. Let $\widetilde{U}(V)$ be the associative algebra attached to a vertex algebra $V$ [FBZ, 4.3.1. Definition]. We have an equivalence of the category of $V$-modules and the category of smooth modules over $\widetilde{U}(V)$.

See [FBZ, $\S \S 4.1-4.3,5.1]$ for the detail. We will give a vertex Poisson analogue of this fact in $\S 4.3 .3$.

4.1.3. Graded vertex superalgebras. Let us cite from [FBZ, 1.3.1] the notion of graded vertex superalgebras.

Definition 4.1.9. A vertex superalgebra $V$ is graded if there is a decomposition $V=\bigoplus_{\Delta \in \mathbb{k}} V_{\Delta}$ as a linear superspace such that the following conditions hold.

(i) $|0\rangle \in V_{0}$.

(ii) $T\left(V_{\Delta}\right) \subset V_{\Delta+1}$ for any $\Delta \in \mathbb{k}$.

(iii) $a_{(n)} b \in V_{-\left(\Delta+\Delta^{\prime}-n-1\right)}$ holds for any $a \in V_{-\Delta}, b \in V_{-\Delta^{\prime}}$ and $n \in \mathbb{Z}$.

Setting $I:=\left\{\Delta \in \mathbb{k} \mid V_{-\Delta} \neq 0\right\}$, we also call $V$ an $I$-graded vertex superalgebra.

Remark 4.1.10. There is an equivalent notion of a vertex superalgebra $V$ with Hamiltonian $H$ [Ka, §4.9]. Such $V$ is equipped with an even linear endomorphism $H$ such that the following conditions hold.

(1) $H$ acts semisimply on $V$.

(2) For any $a, b \in V$ the equation $[H, Y(a, z)] b=\left(Y(H a, z)+z \partial_{z} Y(a, z)\right) b$ in $V((z))$ holds.

Given such $(V, H)$, we have the $H$-eigen decomposition $V=\bigoplus_{\Delta \in \mathbb{k}} V_{\Delta}, V_{\Delta}:=\{a \in V \mid H a=-\Delta a\}$. Setting $I:=\left\{\Delta \in \mathbb{k} \mid V_{\Delta} \neq 0\right\}$, we recover Definition 4.1.9.

Conversely, given an $I$-graded vertex superalgebra $V$, defining $H a:=\Delta a$ for $a \in V_{-\Delta}$ we have a Hamiltonian $H$ on $V$.

We also have the notion of graded modules of graded vsas.

Definition 4.1.11. Let $V$ be a graded vertex superalgebra. A $V$-module $M$ is graded if there is a linear decomposition $M=\oplus_{\Delta \in \mathbb{k}} M_{\Delta}$ such that $a_{(n)}^{M} b \in M_{-\left(\Delta+\Delta^{\prime}-n-1\right)}$ holds for any $a \in V_{-\Delta}, b \in V_{-\Delta^{\prime}}$ and $n \in \mathbb{Z}$. 4.1.4. Universal affine vertex algebras. In this part we recall the universal affine vertex algebra. For simplicity, we work over $\mathbb{C}$. See $[\mathrm{FBZ}, \S 2.4]$ for the detail. We first fix the notation on Lie algebras.

Notation 4.1.12. (1) Let $G$ be a simply connected semisimple algebraic group over $\mathbb{C}$, and $\mathfrak{g}:=$ Lie $G$ be its Lie algebra.

(2) We denote by $\kappa_{\mathfrak{g}}$ the Killing form and by $h^{\vee}$ the dual Coxeter number of $\mathfrak{g}$.

(3) We denote by $\mathfrak{g}((t)):=\mathfrak{g} \otimes_{\mathbb{C}} \mathbb{C}((t))$ the tensor product with the linear space $\mathbb{C}((t))$ of formal Laurent series, and denote its element by $x f:=x \otimes f \in \mathfrak{g}((t))$ with $x \in \mathfrak{g}$ and $f=f(t) \in \mathbb{C}((t))$. Similarly we will denote $\mathfrak{g}[t]:=\mathfrak{g} \otimes_{\mathbb{C}} \mathbb{C}[t], \mathfrak{g}[[t]]:=\mathfrak{g} \otimes_{\mathbb{C}} \mathbb{C}[[t]]$ and so on.

(4) We denote by $\widetilde{\mathfrak{g}}$ the non-twisted affine Kac-Moody Lie algebra associated with $\mathfrak{g}$. It is a Lie algebra of which

- the underlying linear space is $\mathfrak{g}((t)) \oplus \mathbb{C} K \oplus \mathbb{C} D$, and

- the commutation relations are

$$
\left[x t^{m}, y t^{n}\right]=[x, y] t^{m+n}+m \delta_{m+n, 0} \frac{1}{2 h^{\vee}} \kappa_{\mathfrak{g}}(x, y) K, \quad\left[D, x t^{m}\right]=m x t^{m}, \quad[K, \widehat{\mathfrak{g}}]=0
$$

for $x, y \in \mathfrak{g}$. 
(5) We denote by $\widehat{\mathfrak{g}}$ the derived algebra of $\widetilde{\mathfrak{g}}$. Thus we have $\widehat{\mathfrak{g}}=\mathfrak{g}((t)) \oplus \mathbb{C} K$ as a linear space.

Here is the definition of the universal affine vertex algebra.

Definition 4.1.13. For $k \in \mathbb{C}$, we define a $\widetilde{\mathfrak{g}}$-module $V_{k}(\mathfrak{g})$ by

$$
V_{k}(\mathfrak{g}):=U(\widetilde{\mathfrak{g}}) \otimes_{U(\mathfrak{g}[t] \oplus \mathbb{C} K \oplus \mathbb{C} D)} \mathbb{C}_{k},
$$

where $\mathbb{C}_{k}$ is the one-dimensional representation of the Lie subalgebra $\mathfrak{g}[t] \oplus \mathbb{C} K \oplus \mathbb{C} D \subset \widetilde{\mathfrak{g}}$ on which $\mathfrak{g}[t] \oplus \mathbb{C} D$ acts trivially and $K$ acts as multiplication by $k$, Then $V_{k}(\mathfrak{g})$ is a vertex algebra of which

- the vacuum vector is $|0\rangle=1 \otimes 1$,

- the translation is $T=-\partial_{t}$, and

- $Y\left(x t^{-1}|0\rangle, z\right)=x(z):=\sum_{n \in \mathbb{Z}}\left(x t^{n}\right) z^{-n-1}$ for $x \in \mathfrak{g}$.

These conditions determines the vertex algebra structure uniquely. It is $\mathbb{N}$-graded by the Hamiltonian $-D$ (Remark 4.1.10). We call the obtained vertex algebra $V_{k}(\mathfrak{g})$ the universal affine vertex algebra associated with $\mathfrak{g}$ at level $k$.

Remark. We used the normalization of invariant bilinear form of [FBZ]. Comparing it with the notation $V^{\kappa}(\mathfrak{g})$ in $[\mathrm{A}]$ where $\kappa$ denotes a bilinear invariant form on $\mathfrak{g}$, we have $V_{1}(\mathfrak{g})=V^{\kappa_{\mathfrak{g}}}(\mathfrak{g})$ and $V_{-h^{\vee}}(\mathfrak{g})=V^{\kappa_{c}}(\mathfrak{g})$ with $\kappa_{c}:=-\frac{1}{2} \kappa_{\mathfrak{g}}$. By the latter relation, $\kappa_{c}$ is called the critical level in [A].

For later use, we recall:

Definition 4.1.14. Let $R$ be a associative superalgebra. Let also $a(z)=\sum_{n \in \mathbb{Z}} a_{n} z^{n}$ and $b(z)=\sum_{n \in \mathbb{Z}} b_{n} z^{n}$ be formal power series with coefficients $a_{n}$ and $b_{n}$ in $R$ such that the parity of the coefficients are constant for each series. We denote $p(a):=p\left(a_{n}\right)$ and $p(b):=p\left(b_{n}\right)$. Then the normal ordering $: a(z) b(z):$ of $a(z)$ and $b(z)$ is defined to be the formal power series

$$
: a(z) b(z)::=a(z)_{+} b(z)+(-1)^{p(a) p(b)} b(z) a(z)_{-},
$$

where we set $a(z)_{+}:=\sum_{n \geq 0} a_{(n)} z^{n}$ and $a(z)_{-}:=\sum_{n \leq-1} a_{(n)} z^{n}$.

Fact 4.1.15 $([\mathrm{FBZ}, \S 2.4])$. Let $\left\{x_{i} \mid i=1, \ldots, \operatorname{dim} \mathfrak{g}\right\}$ be a linear basis of $\mathfrak{g}$. Then

(1) $V_{k}(\mathfrak{g})$ has a linear basis of monomials of the form

$$
v=\left(x_{i_{1}} t^{n_{1}}\right) \cdots\left(x_{i_{l}} t^{n_{l}}\right)|0\rangle,
$$

where $n_{i} \in \mathbb{Z}, n_{1} \leq \cdots \leq n_{l}<0$ and if $n_{j}=n_{j+1}$ then $i_{j} \leq i_{j+1}$.

(2) For the element $v \in V_{k}(\mathfrak{g})$ in the previous item, we have

$$
Y(v, z)=\frac{1}{\left(-n_{1}+1\right) !} \cdots \frac{1}{\left(-n_{l}+1\right) !}: \partial_{z}^{-n_{1}+1} x_{i_{1}}(z) \cdots \partial_{z}^{-n_{l}+1} x_{i_{l}}(z): .
$$

Next we consider the category $V_{k}(\mathfrak{g})$-VMod of modules over the universal affine vertex algebra $V_{k}(\mathfrak{g})$. By Fact 4.1 .8 , it is equivalent to the smooth modules over the associative algebra $\widetilde{U}\left(V_{k}(\mathfrak{g})\right)$ attached to $V_{k}(\mathfrak{g})$. Denoting by $\widetilde{U}_{k}(\widehat{\mathfrak{g}})$ the $t$-adic completion of $U_{k}(\widehat{\mathfrak{g}}):=U(\widehat{\mathfrak{g}}) /(K-k)$, we have $\widetilde{U}\left(V_{k}(\mathfrak{g})\right) \simeq \widetilde{U}_{k}(\widehat{\mathfrak{g}})$ by $[\mathrm{FBZ}$, 4.3.2. Lemma]. Then a smooth $\widetilde{U}\left(V_{k}(\mathfrak{g})\right)$-module is nothing but a smooth $\mathfrak{g}_{k}$-module. In other words, we have:

Fact 4.1.16 ([FBZ, 5.1.8]). A $V_{k}(\mathfrak{g})$-module is equivalent to a smooth $\widehat{\mathfrak{g}}$-module of level $k$, i.e., a representation $M$ of the Lie algebra $\widehat{\mathfrak{g}}$ such that $K$ acts by multiplication $k$ and $\left(x t^{n}\right) \cdot m=0$ for any $x \in \mathfrak{g}, m \in M$ and $n \gg 0$.

Recall that there is a monoidal structure $\otimes$ on the category of representations of a Lie algebra $L$, which is induced by the comultiplication $\Delta(x)=x \otimes 1+1 \otimes x$ for each element $x \in L$. We call this action on the tensor the diagonal action of $L$. Together with Fact 4.1.16, we have the following tensor structure on $V_{k}(\mathfrak{g})$-modules with different $k$ 's:

Lemma 4.1.17. Let $k, k^{\prime} \in \mathbb{C}, M \in V_{k}(\mathfrak{g})$-VMod and $N \in V_{k^{\prime}}(\mathfrak{g})$-VMod. Then the tensor product $M \otimes N$ in $\mathrm{Vec}$ is an object of $V_{k+k^{\prime}}(\mathfrak{g})$-VMod by the diagonal action of $\widehat{\mathfrak{g}}$.

4.1.5. Dg vertex algebras. Now we introduce a $\mathrm{dg}$ analogue of a vertex algebra. There are in fact several choices of definition, and we give one suitable for our purpose. Let us first recall

Definition 4.1.18 $([\mathrm{Ka}, \S 4.3])$. Let $V$ be a vertex superalgebra, and let $\varepsilon \in \mathbb{Z} / 2 \mathbb{Z}$. A derivation $d$ of parity $\varepsilon$ on $V$ is a linear endomorphism of parity $\varepsilon$, i.e., $d \in \operatorname{End}_{\mathbb{k}}(V)_{\varepsilon}$, such that for any $a \in V$ we have

$$
[d, Y(a, z)]=Y(d a, z) .
$$


Remark 4.1.19. Let $d$ be a derivation of a vertex superalgebra $(V,|0\rangle, T, Y)$.

(1) We have $[d, T]=0$ by Remark 4.1.6 (2).

(2) We have $d|0\rangle=0$ by Remark 4.1.6 (3).

(3) The condition on $d$ and $Y$ is equivalent to $d\left(a_{(n)} b\right)=(d a)_{(n)} b+(-1)^{p(a) \varepsilon} a_{(n)}(d b)$ for any $a, b \in V$ with $a$ of pure parity.

Here is our definition of a dg vertex algebra:

Definition 4.1.20. (1) A dg vertex algebra (dgva for short) is a complex $\left(V^{\bullet}, d\right)$ equipped with a vertex superalgebra structure $(|0\rangle, T, Y)$ on the associated linear superspace $V^{\text {even }} \oplus V^{\text {odd }}$ (Notation 4.1.4) such that the following conditions hold.

(i) $|0\rangle \in V^{0}$ and $T \in \underline{\operatorname{End}}(V)^{0}=\operatorname{Hom}_{\mathrm{dgVec}}(V, V)$.

(ii) $d$ is an odd derivation (Definition 4.1.18) of the vertex superalgebra ( $\left.V^{\text {even }} \oplus V^{\text {odd }},|0\rangle, T, Y\right)$.

(iii) The state-field correspondence $Y$ is homogeneous. In other words, we have $a_{(n)} V^{j} \subset V^{i+j}$ for any $a \in V^{i}$ and $n \in \mathbb{Z}$.

We denote a dg vertex superalgebra as $V=\left(V^{\bullet}, d,|0\rangle, T, Y\right)$.

(2) We also have the notions of morphisms and tensor products of dg vertex algebras. We denote by dgVA the category of dg vertex algebras.

For the later use, let us also introduce the corresponding notion of dg modules.

Definition 4.1.21. Let $V$ be a dg vertex algebra.

(1) A $d g V$-module $M=\left(M^{\bullet}, d_{M}, Y^{M}\right)$ consists of

- a complex $\left(M^{\bullet}, d_{M}\right)$ and

- a $\left(V^{\text {even }} \oplus V^{\text {odd }}\right)$-module structure on $M^{\text {even }} \oplus M^{\text {odd }}, Y^{M}(a, z) m=\sum_{n \in \mathbb{Z}} a_{(n)}^{M} m z^{-n-1}$, in the sense of Definition 4.1.11, where $V^{\text {even }} \oplus V^{\text {odd }}$ is regarded as a vertex superalgebra, such that

$$
a_{(n)}^{M} M^{j} \subset M^{|a|+j}, \quad d_{M}\left(a_{(n)}^{M} m\right)=\left(d_{V} a\right)_{(n)}^{M} m+(-1)^{|a|} a_{(n)}^{M}\left(d_{M} m\right)
$$

for any homogeneous $a \in V$, any $m \in M$ and any $j, n \in \mathbb{Z}$.

(2) We denote by $V$-dgVMod the category of dg $V$-modules. For a $\operatorname{dg} V$-module $M$, the $\mathbb{Z}$-grading of the underlying complex $\left(M^{\bullet}, d_{M}\right)$ is called the cohomological degree.

As for the cohomology of a dg vertex algebra, we have:

Lemma 4.1.22. For a dg vertex algebra $V=\left(V^{\bullet}, d,|0\rangle, T, Y\right)$, consider the cohomology $H^{\bullet}=H\left(V^{\bullet}, d\right)$ of the underlying complex (Definition 1.1.7). Then the associated linear superspace $H^{\text {even }} \oplus H^{\text {odd }}$ (Notation 4.1.4) has a structure of vertex superalgebra.

Proof. The argument in [FBZ, §5.7.3] works. For the completeness, let us write down it. By Remark 4.1.19 (1), the translation $T$ preserves $\operatorname{Ker} d$ and $\operatorname{Im} d$. The derivation property $[d, Y(a, z)]=Y(d a, z)$ yields that Ker $d$ is a vertex sub-superalgebra and $\operatorname{Im} d$ is a vsa ideal of $V$. Thus we have a quotient vsa $H\left(V^{\bullet}, d\right)$.

The cohomology has a $\mathbb{Z}$-grading $H^{\bullet}\left(V^{\bullet}, d\right)$ induced by the cohomological degree $V^{\bullet}$. But it is not necessarily a graded vertex superalgebra in the sense of Definition 4.1.9. In other words, the $\mathbb{Z}$-grading may not be given by the eigenvalue of any Hamiltonian. In order to avoid this conflict, we introduce

Definition 4.1.23. A graded dg vertex algebra is a $d g$ vertex algebra $V$ equipped with an additional linear decomposition $V=\oplus_{\Delta \in \mathbb{k}} V_{\Delta}$ such that the following conditions hold.

(i) The underlying vsa $\left(V^{\text {even }} \oplus V^{\text {odd }},|0\rangle, T, Y\right)$ is graded in the sense of Definition 4.1.9.

(ii) $d$ preserves the additional gradings.

A motivational example is weak BRST complex of vertex algebras in [A07, 3.15]. We will give explicit examples in $\S 4.1 .6$.

Similarly as Definition 4.1.11, we also have the notion of graded $d g V$-modules of a graded dg vertex algebra $V$. We then have an obvious analogue of Example 4.1.7:

Lemma 4.1.24. Let $V$ and $W$ be graded dg vertex algebras and $\varphi: V \rightarrow W$ be a morphism of dg vertex algebras which preserves the additional gradings. Then $W$ is naturally a graded $\operatorname{dg} V$-module. 
4.1.6. The free fermionic vertex algebra. Let us recall the $d g$ vertex algebra of free fermions, a standard example of dg vertex algebra. See [FBZ, 4.3.1] for more information. See also [BD, 3.8.6], where the coordinate free version is introduced under the name chiral Clifford algebra.

Let $U$ be a bounded complex, and $U^{*}:=\underline{\operatorname{Hom}}(U, \mathbb{k})$ be its dual. We have the canonical pairing $\langle\cdot, \cdot\rangle$ : $U^{*} \otimes U \rightarrow \mathbb{k}$. We denote by $\mathbb{k}((t)) d t$ the linear space of one-forms on formal Laurent series. We have the residue pairing $(f(t), g(t) d t) \mapsto \operatorname{Res}_{t}(f(t) g(t) d t)$ on $\mathbb{k}((t)) \otimes \mathbb{k}((t)) d t$. Thus, denoting $U((t)):=U \otimes \mathbb{k}((t))$ and $U^{*}((t)) d t:=U \otimes \mathbb{k}((t)) d t$, we have a skew-symmetric pairing $(\cdot, \cdot)$ on $U((t))[1] \oplus U^{*}((t)) d t[-1]$ and itself induced by $\langle\cdot, \cdot\rangle$ and $\operatorname{Res}_{t}$.

For the pair $U((t))[1] \oplus U^{*}((t)) d t[-1]$ and $(\cdot, \cdot)$, we denote the associated Clifford algebra by

$$
\mathrm{Cl}=\mathrm{Cl}\left(U((t))[1] \oplus U^{*}((t))[-1],(\cdot, \cdot)\right) .
$$

It is a complete topological unital dg algebra. Explicitly, taking a homogeneous linear basis $\left\{u_{i} \mid i \in I\right\}$ of $U$ and the dual basis $\left\{u_{i}^{*} \mid i \in I\right\}$ of $U^{*}$, we have that $\mathrm{Cl}$ is generated by

$$
\psi_{i, n}:=\left(u_{i} \otimes t^{n}\right)[1] \in U((t))[1], \quad \psi_{i, n}^{*}:=\left(u_{i}^{*} \otimes t^{n-1} d t\right)[-1] \in U^{*}((t)) d t[-1] \quad(i \in I, n \in \mathbb{Z})
$$

and they satisfy

$$
\left[\psi_{i, m}, \psi_{j} t^{n}\right]=\left[\psi_{i}^{*} t^{m-1} d t, \psi_{j}^{*} t^{n-1} d t\right]=0, \quad\left[\psi_{i} t^{m}, \psi_{j}^{*} t^{n-1} d t\right]=\delta_{i, j} \delta_{m,-n}
$$

Let us denote by $\bigwedge^{\frac{\infty}{2}}(U)$ the fermionic Fock module of $\mathrm{Cl}$. It is a left $\mathrm{dg}$ Cl-module generated by an element $|0\rangle$ such that

$$
\psi_{i} t^{m}|0\rangle=0(m \geq 0), \quad \psi_{i}^{*} t^{n-1} d t|0\rangle=0 \quad(n \geq 1) .
$$

It has a homogeneous basis consisting of the elements of the form

$$
v=\left(\psi_{i_{1}} t^{m_{1}}\right) \cdots\left(\psi_{i_{k}} t^{m_{k}}\right)\left(\psi_{j_{1}}^{*} t^{n_{1}-1} d t\right) \cdots\left(\psi_{j_{l}}^{*} t^{n_{l}-1} d t\right)|0\rangle \quad\left(m_{1}<\cdots<m_{k}<0, n_{1}<\cdots<n_{l} \leq 0\right) .
$$

We have an additional $\mathbb{Z}$-grading to the dg structure, called the charge grading, under which the vector (4.2) is attached with charge $=-k+l$. We denote $\bigwedge^{\frac{\infty}{2}+\bullet}(U)$ to emphasize the charge grading.

If $U$ is concentrated in degree 0 , then the charge is equal to the minus of the cohomological degree, and the parity is given by the charge modulo 2 . In this case $\bigwedge^{\frac{\infty}{2}}(U)$ is also called the space of semi-infinite wedges.

We now recall:

Definition 4.1.25. The fermionic Fock module $\bigwedge^{\frac{\infty}{2}}(U)$ has a structure of dg vertex algebra with Hamiltonian, where

- the vacuum is given by $|0\rangle$,

- the translation is given by $T=-\partial_{t}$, and

- the state-field correspondence is given by

$$
\begin{aligned}
& Y\left(\psi_{i} t^{-1}|0\rangle, z\right)=\psi_{i}(z):=\sum_{n \in \mathbb{Z}}\left(\psi_{i} t^{n}\right) z^{-n-1}, \\
& Y\left(\psi_{i}^{*} t^{-1} d t|0\rangle, z\right)=\psi_{i}^{*}(z):=\sum_{n \in \mathbb{Z}}\left(\psi_{i}^{*} t^{n-1} d t\right) z^{-n},
\end{aligned}
$$

and for the element $v=\left(\psi_{i_{1}} t^{m_{1}}\right) \cdots\left(\psi_{i_{k}} t^{m_{k}}\right)\left(\psi_{j_{1}}^{*} t^{n_{1}-1} d t\right) \cdots\left(\psi_{j_{l}}^{*} t^{n_{l}-1} d t\right)|0\rangle$ in (4.2), it is

$$
Y(v, z)=\prod_{a=1}^{k} \frac{1}{\left(-m_{a}-1\right) !} \prod_{b=1}^{l} \frac{1}{\left(-n_{b}-1\right) !}: \partial_{z}^{-m_{1}-1} \psi_{i_{1}}(z) \cdots \partial_{z}^{-m_{k}-1} \psi_{i_{k}}(z) \partial_{z}^{-n_{1}} \psi_{j_{1}}^{*}(z) \cdots \partial_{z}^{-n_{l}} \psi_{j_{l}}^{*}(z):
$$

where we used the normal ordering (Definition 4.1.14).

- The additional $\mathbb{Z}$-grading is given by the charge grading.

We call it the free fermionic vertex algebra, or the chiral Clifford algebra and denote by

$$
\bigwedge^{\frac{\infty}{2}}(U)=\mathrm{Cl}_{\mathrm{ch}}\left(U((t)), U^{*}((t)) d t,(\cdot, \cdot)\right) .
$$

For the second notation, see Remark 6.1.1

4.2. $\mathbf{D g}$ vertex $\mathbf{L i}$ algebras. In this subsection we recall the notion vertex Lie algebras. It is designed to encode the "polar part" of vertex algebra. We work over a field $\mathbb{k}$ of characteristic 0. 


\subsubsection{Definition.}

Definition 4.2.1. (1) A dg vertex Lie algebra is a data $\left(L, d, T, Y_{-}\right)$consists of

- a complex $(L, d)$,

- an endomorphism $T \in \underline{\operatorname{End}}(L)^{0}=\operatorname{Hom}_{\mathrm{dgVec}}(L, L)$, and

- a morphism $L \otimes L \rightarrow z^{-1} L\left[z^{-1}\right]$ of complexes, $a \otimes b \mapsto Y_{-}(a, z) b=\sum_{n \in \mathbb{N}}\left(a_{(n)} b\right) z^{-n-1}$ satisfying the following conditions.

(i) $Y_{-}(T a, z)=\partial_{z} Y_{-}(a, z)$.

(ii) For any homogeneous $a, b \in L$, we have

$$
Y_{-}(a, z) b=\left((-1)^{|a||b|} e^{z T} Y_{-}(b,-z) a\right)_{-} .
$$

Here for a formal Laurent series $f(z)=\sum_{i} f_{i} z^{i} \in L((z))$ we set $f(z)_{-}:=\sum_{i<0} f_{i} z^{i} \in$ $z^{-1} L\left[z^{-1}\right]$.

(iii) For $a, b \in L$ we have $\left[a_{(m)}, b_{(n)}\right]=\sum_{i \in \mathbb{N}}\left(\begin{array}{c}m \\ i\end{array}\right)\left(a_{(i)} b\right)_{(m+n-i)}$ in $\underline{\operatorname{End}}(L)=\underline{\operatorname{Hom}}_{\mathrm{dgVec}}(L, L)$.

(iv) For any $a, b \in L$, we have $\left[d, Y_{-}(a, z)\right] b=Y_{-}(d a, z) b$.

(2) We have obvious notions of morphism and tensor product of dg vertex Lie algebras. We denote by $\mathrm{dg} V \mathrm{~L}$ the category of dg vertex Lie algebras.

Similarly we have the notions of vertex Lie superalgebra and vertex Lie algebra. We refer [FBZ, §16.1] for the detail of the latter notion.

Remark 4.2.2. (1) The condition (ii) is equivalent to

$$
a_{(n)} b=\sum_{i \in \mathbb{N}}(-1)^{|a||b|+n+i+1} \frac{1}{i !} T^{i}\left(b_{(n+i)} a\right) \quad(n \in \mathbb{N}) .
$$

(2) We can check that the quotient space $L / \operatorname{Im}(T)$ is a dg Lie algebra with the Lie bracket

$$
[\bar{a}, \bar{b}]:=\overline{a_{(0)} b}
$$

for $a, b \in L$. Indeed, by the condition (i) we have $(T a)_{(0)} b=0$ so that the Lie bracket is well-defined, by the condition (ii) and the previous item (1) we have $a_{(0)} b=-(-1)^{|a||b|} b_{(0)} a \bmod \operatorname{Im}(T)$ so that the anti-commutativity holds, and by (iii) we have $\left[a_{(0)}, b_{(0)}\right]=\left(a_{(0)} b\right)_{(0)}$ and can check the Jacobi relation by direct computation.

(3) By the theory of coisson algebras [BD, §2.6], a vertex Lie algebra satisfying some conditions can be regarded as a Lie algebra object in a certain symmetric monoidal category. See Remark 5.1.1 for more information.

By the definition we immediately have the following construction of vertex Lie algebras from vertex algebras. See [FBZ, 16.1.2-3] for the detail.

Lemma 4.2.3. For a dg vertex algebra $V=(V, d,|0\rangle, T, Y)$, we have a $\mathrm{dg}$ vertex Lie algebra

$$
V_{\text {Lie }}:=\left(V, d, T, Y_{-}\right)
$$

by setting $Y_{-}(a, z):=Y(a, z)=\sum_{n \in \mathbb{N}} a_{(n)} z^{-n-1}$. This construction gives rise to a functor

$$
\mathrm{dgVA} \longrightarrow \mathrm{dgVL}, \quad V \longmapsto V_{\text {Lie }},
$$

which we call the polar part construction.

See $§ 4.2 .2$ for the adjoint of the polar part construction. For later citation, we give an example.

Example 4.2.4. We use the notations in $\S 4.1 .4$ here. Consider the universal affine vertex algebra $V_{k}(\mathfrak{g})$ over $\mathbb{C}$. Following $[\mathrm{FBZ}, 16.1 .5]$, we denote by

$$
v_{k}(\mathfrak{g}) \subset V_{k}(\mathfrak{g})
$$

the linear subspace spanned by the vacuum vector $|0\rangle$ and the vectors $x t^{m}|0\rangle\left(x \in \mathfrak{g}, m \in \mathbb{Z}_{<0}\right)$. Thus we have $v_{k}(\mathfrak{g}) \simeq \mathfrak{g}\left[t^{-1}\right] \oplus \mathbb{C}$ as linear spaces. The subspace $v_{k}(\mathfrak{g})$ is invariant under the action of $x_{-}(z):=$ $Y\left(x t^{-1}|0\rangle, z\right)_{-}=\sum_{n \in \mathbb{N}}\left(x t^{n}\right) z^{-n-1}$, and we can see that $\left(v_{k}(\mathfrak{g}), T, Y_{-}\right)$with $Y_{-}\left(x t^{-1}|0\rangle, z\right):=x_{-}(z)$ is a vertex Lie algebra.

Here is another construction of dg vertex Lie algebras:

Lemma 4.2.5. Let $\mathfrak{l}$ be dg Lie algebra, and consider $\mathfrak{l}[[t]]=\mathfrak{l} \otimes \mathbb{k}[[t]]$. It is a dg Lie algebra with the Lie bracket $[x \otimes f, y \otimes g]:=[x, y] \mathfrak{r} \otimes(f g)$ for $x, y \in \mathfrak{l}$ and $f, g \in \mathbb{k}[[t]]$. Then $\mathfrak{l}[[t]]$ has the following dg vertex Lie algebra structure:

- The translation $T$ is given by the multiplication by $t$. Thus $T\left(x \otimes t^{i}\right)=x \otimes t^{i+1}$. 
- The operation $Y_{-}$is determined by

$$
\begin{aligned}
& Y_{-}(x \otimes 1, z)=x_{-}(z):=\sum_{n \in \mathbb{N}} z^{-n-1} \partial_{t}^{n}([x,-]), \\
& Y_{-}\left(x \otimes t^{i}, z\right)\left(y \otimes t^{j}\right):=\left((-1)^{|x||y|} e^{z t}\left(-\partial_{z}\right)^{j} y_{-}(-z)\left(x \otimes t^{i}\right)\right)_{-} .
\end{aligned}
$$

Explicitly, we have

$$
\left(x \otimes t^{i}\right)_{(n)}\left(y \otimes t^{j}\right)=(-1)^{i} \frac{n !}{(n-i) !} \frac{j !}{(j-n+i) !}[x, y]_{\mathfrak{l}} \otimes t^{i+j-n}
$$

for $i \leq n \leq i+j$ and 0 otherwise.

We denote the obtained dg vertex Lie algebra by

$$
J_{\infty}(\mathfrak{l}):=\left(\mathfrak{l}[[t]], d_{\mathfrak{l}}, T, Y_{-}\right),
$$

and call it the level 0 dg vertex Lie algebra.

Proof. It is enough to check the conditions (i)-(iv) in Definition 4.2.1. The condition (i) follows from (4.4). The condition (ii) follows from the definition (4.3). The condition (iii) can be checked with the help of Jacobi relation of $\mathfrak{l}$. The condition (iv) follows from (4.4) and the dg Lie algebra structure on $\mathfrak{l}$.

For the naming "level 0", see Example 4.3.10.

4.2.2. Enveloping vertex algebra. We explain the universal construction of dg vertex algebra from a dg vertex Lie algebra, following the non-dg version in [FBZ, §16.1]. See also [BD, §3.7] for the chiral algebra version.

Let $L=\left(L, d, T, Y_{-}\right)$be a dg vertex Lie algebra, and consider the operator $\partial:=T \otimes \mathrm{id}+\mathrm{id} \otimes \partial_{t}$ on $L((s))=L \otimes \mathbb{k}((s))$. We define

$$
\operatorname{Lie}(L):=L((s)) / \operatorname{Im}(\partial),
$$

which inherits the dg structure of $L$. We also denote $x_{[n]}:=\overline{x \otimes s^{n}} \in \operatorname{Lie}(L)$ for $x \in L$ and $n \in \mathbb{Z}$, and define

$$
\operatorname{Lie}(L)+\subset \operatorname{Lie}(L)
$$

to be the subspace which is the completion of the linear span of $x_{[n]}$ with $n \in \mathbb{N}$. It is in fact a subcomplex of $\operatorname{Lie}(L)$.

Lemma $([\mathrm{FBZ}, 16.1 .7$. Lemma]). (1) $\operatorname{Lie}(L)$ is a dg Lie algebra with the Lie bracket

$$
\left[x_{[m]}, y_{[n]}\right]:=\sum_{l \in \mathbb{N}}\left(\begin{array}{c}
m \\
l
\end{array}\right)\left(x_{(l)} y\right)_{[m+n-l]},
$$

where we denoted by $Y_{-}(x, z)=\sum_{n \in \mathbb{N}} x_{(n)} z^{-n-1}$ the vertex Lie structure of $L$.

(2) $\operatorname{Lie}(L)_{+}$is a dg Lie subalgebra of $\operatorname{Lie}(L)$.

(3) The correspondence $\operatorname{Lie}(L)_{+} \rightarrow \underline{\operatorname{End}}(L), x_{[n]} \rightarrow x_{(n)}$ gives a morphism of dg Lie algebras.

Consider the universal enveloping algebra $U(\operatorname{Lie}(L))$ and $U\left(\operatorname{Lie}(L)_{+}\right)$of the dg Lie algebras in the above lemma. We define a left $\operatorname{dg} U(\operatorname{Lie}(L))$-module

$$
U(L):=U(\operatorname{Lie}(L)) \otimes_{U\left(\operatorname{Lie}(L)_{+}\right)} \mathbb{k},
$$

where $\mathbb{k}$ denotes the trivial one-dimensional representation of $U\left(\operatorname{Lie}(L)_{+}\right)$.

Proposition ([FBZ, 16.1.12. Propsition]). There is a unique dg vertex algebra structure on $U(L)$ such that

- the vacuum is $|0\rangle:=1 \otimes 1$,

- the translation $T$ is defined by $T|0\rangle=0$ and $\left[T, x_{[n]}\right]=-n x_{[n-1]}$, and

- $Y\left(x_{[-1]}|0\rangle, z\right)=\sum_{n \in \mathbb{Z}} x_{[n]} z^{-n-1}$ for $x \in L$.

Moreover, the correspondence $L \mapsto U(L)$ gives a functor that is a left adjoint of the polar part construction $V \mapsto V_{\text {Lie }}$ in Lemma 4.2.3:

$$
(-)_{\text {Lie }}: \operatorname{dgVA} \rightleftarrows \mathrm{dgVL}: U(-) .
$$

We call $U(L)$ the enveloping vertex algebra of $L$.

Finally let us explain a twisted version of $U(L)$. See [BD, 3.7.20] for the original definition. We note that the category $d g V L$ of dg vertex algebras is an abelian category.

Definition 4.2.6. Let $L$ be a dg vertex Lie algebra. 
(1) A one-dimensional central extension of $L$ is a dg vertex Lie algebra $L^{b}$ which sits in an exact sequence

$$
0 \longrightarrow \mathbb{k}_{\text {Lie }} \longrightarrow L^{b} \longrightarrow L \longrightarrow 0
$$

in the abelian category dgVL. Here $\mathbb{k}_{\text {Lie }}$ denotes the one-dimensional linear space with trivial dg vertex Lie algebra structure. We denote by $1^{b} \in L^{b}$ the image of $1_{\mathbb{k}} \in \mathbb{k}_{\text {Lie }}$.

(2) Assume that we are given a one-dimensional central extension $L^{b}$ of $L$. We define

$$
U(L)^{b}:=U\left(L^{b}\right) /\left(1_{U(L)}-1^{b}\right),
$$

where $1^{b}$ denotes the image of $1^{b} \in L^{b}$ in the enveloping vertex algebra $U\left(L^{b}\right)$. This quotient inherits the dg vertex algebra structure of $U\left(L^{b}\right)$. We call the resulting dg vertex algebra $U\left(L^{b}\right)$ the twisted enveloping vertex algebra of $L$.

(3) $U(L)$ and $U(L)^{b}$ inherit the PBW filtration of $U(\operatorname{Lie}(L))$ and $U\left(\operatorname{Lie}\left(L^{b}\right)\right)$ respectively. We call the resulting filtration the $P B W$ filtration of the (twisted) enveloping vertex algebra, and denote it by $U(L)_{\bullet}, U(L)_{\bullet}^{b}$.

Example 4.2.7 ([FBZ, 16.1.9]). Consider the vertex Lie algebra $v_{k}(\mathfrak{g})$ over $\mathbb{C}$ in Example 4.2.4. Recalling that $v_{k}(\mathfrak{g})=\mathfrak{g}\left[t^{-1}\right]|0\rangle \oplus \mathbb{C}|0\rangle$ as linear spaces, we can check that $\operatorname{Lie}\left(v_{k}(\mathfrak{g})\right)$ is spanned by $\left(x t^{-1}|0\rangle\right)_{[n]}$ $(n \in \mathbb{Z})$ and $|0\rangle_{[-1]}$, and the commutation relation is $\left[\left(x t^{-1}|0\rangle\right)_{[m]},\left(y t^{-1}|0\rangle\right)_{[n]}\right]=\left([x, y]_{\mathfrak{g}} t^{-1}|0\rangle\right)_{[m+n]}+$ $\frac{k}{2 h^{\vee}} m \delta_{m+n, 0} \kappa_{\mathfrak{g}}(x, y)|0\rangle_{[-1]}$. Thus we have

$$
\operatorname{Lie}\left(v_{k}(\mathfrak{g})\right) \simeq \widehat{\mathfrak{g}}_{k}, \quad \operatorname{Lie}\left(v_{k}(\mathfrak{g})\right)_{+} \simeq \mathfrak{g}[[t]]
$$

where $\widehat{\mathfrak{g}}_{k}=\mathfrak{g}((t)) \oplus \mathbb{C} \mathbf{1}$ denotes the affine Lie algebra obtained by replacing $K$ in $\widehat{\mathfrak{g}}$ with $k \mathbf{1}$. Thus $U\left(v_{k}(\mathfrak{g})\right) \simeq$ $U(\widehat{\mathfrak{g}}) \otimes_{U(\mathfrak{g}[[t]])} \mathbb{C}$.

The linear space $L:=\mathfrak{g}\left[t^{-1}\right]$ has a structure of vertex Lie algebra induced by the Lie algebra $\mathfrak{g}((t))$. Explicitly, $L$ is regarded as a subspace of the $\mathfrak{g}((t))$-module $U(\mathfrak{g}((t))) \otimes_{U(\mathfrak{g}[[t]])} \mathbb{C}$, and the vertex Lie structure is given by $T=-\partial_{t}$ and $Y_{-}\left(x t^{-1}, z\right)=\sum_{n \in \mathbb{N}}\left(x t^{n}\right) z^{-n-1}$. We have $\operatorname{Lie}(L) \simeq \mathfrak{g}((t))$ and $\operatorname{Lie}(L)_{+} \simeq \mathfrak{g}[[t]]$.

Then $v_{k}(\mathfrak{g})$ is a one-dimensional central extension of $L$ :

$$
0 \longrightarrow \mathbb{C}_{\text {Lie }} \longrightarrow L^{b}=v_{k}(\mathfrak{g}) \longrightarrow L=\mathfrak{g}\left[t^{-1}\right] \longrightarrow 0 .
$$

We can identify $1^{b}=\mathbf{1}$, so that we have $U(L)^{b}=U\left(v_{k}(\mathfrak{g})\right) /\left(1-1^{b}\right) \simeq V_{k}(\widehat{\mathfrak{g}})$.

4.3. Dg vertex Poisson algebras. Recall that a vertex Poisson algebra is an analogue of Poisson algebra in the category of vertex algebras. As Poisson algebra is defined to be a combined structure commutative algebra and Lie algebra, the definition of vertex Poisson algebra is a combination of commutative vertex algebra and vertex Lie algebra. A vertex Poisson algebra is also regarded as a "classical limit" of a vertex algebra, as from a Poisson algebra can be obtained from the PBW filtration of the universal enveloping algebra of a Lie algebra. We refer [FBZ, Chap. 16] for the detail of vertex Poisson algebras.

In this subsection we introduce a $d g$ analogue of vertex Poisson algebras. It is in fact a special case of coisson algebras discussed in [BD, §2.6]. See also Remark 5.1.1 for more information on this point.

4.3.1. Commutative $d g$ vertex algebras. First we introduce a dg version of commutative vertex algebras.

Definition. A dg vertex algebra $V$ is commutative if the integer $N$ in the locality axiom (Definition 4.1.5) can be taken to be 0 for any homogeneous $a, b \in V$. In other words, we have $\left[a_{(m)}, b_{(n)}\right]=0$ for any $m, n \in \mathbb{Z}$.

We can see that for a commutative dg vertex algebra $V$, the state-field correspondence satisfies $a_{(n)}=0$ for any $n \in \mathbb{N}$ and $a \in V$. Hereafter we denote a commutative dg vertex algebra by

$$
\left(V, d,|0\rangle, T, Y_{+}\right)
$$

in order to emphasize $Y_{+}(a, z)=\sum_{n \in \mathbb{Z}_{<0}} a_{(n)} z^{-n-1} \in \underline{\operatorname{End}}(V)[[z]]$.

Now recall the notion of derivations on a dg algebra (Definition 1.2.10). The non-dg version of the next statement is due to Borcherds:

Lemma 4.3.1. A commutative dg vertex algebra $\left(V, d,|0\rangle, T, Y_{+}\right)$is equivalent to a unital commutative dg algebra $(V, d, \cdot,|0\rangle)$ equipped with an extra 0-derivation $T$ commuting with the differential $d$.

Proof. As in the non-dg case (see [FBZ, §1.4]), we attach to a commutative vertex algebra structure $Y_{+}$ the multiplication $a \cdot b:=a_{(-1)} b$, and attach to the differential algebra structure $(\cdot, T)$ the vertex algebra structure $Y_{+}(a, z) b:=\left(e^{z T} a\right)$.

By this lemma, we can denote the commutative dgva by (4.5) or by

$$
(V, d,|0\rangle, T, \cdot) \text {. }
$$


4.3.2. $D g$ vertex Poisson algebras. We turn to the definition of a dg vertex Poisson algebra. Recall that we denote by $\operatorname{Der}(R)^{n}$ the linear space of $n$-derivations for a dg algebra $R$ (Definition 1.2.10). For a commutative dgva $(P, d,|0\rangle, T, \cdot)$, we denote by $\operatorname{Der}(P, \cdot)^{n}$ the corresponding space for the $\operatorname{cdga}(P, \cdot)$. Using this notation, we have:

Definition 4.3.2. (1) A $d g$ vertex Poisson algebra ( $d g$ vpa for short) is a complex $(P, d)$ equipped with

- a structure $\left(P, d,|0\rangle, T, Y_{+}\right)$of commutative dgva, or equivalently, a structure $(P, d,|0\rangle, T, \cdot)$ of unital cdga, and

- a structure $\left(P, d, T, Y_{-}\right)$of dg vertex Lie algebra

such that for any homogeneous $a \in P$ we have

$$
Y_{-}(a, z) \in\left(\operatorname{Der}(P, \cdot)^{|a|}\right)\left[\left[z^{-1}\right]\right] z^{-1} .
$$

We denote it by $\left(P, d,|0\rangle, T, \cdot Y_{-}\right)$or by $\left(P, d,|0\rangle, T, Y_{+}, Y_{-}\right)$, or just by $P$.

(2) Let $V$ be a dg vpa and $a \in V$. We define $a_{(n)}$ for $n \in \mathbb{Z}$ in the following way.

- For $n \geq 0$, we denote by $Y_{-}(a, z)=\sum_{n>0} a_{(n)} z^{-n-1}$ the vertex Lie algebra structure.

- For $n<0$, we denote by $Y_{+}(a, z)=\sum_{n<0} a_{(n)} z^{-n-1}$ the commutative vertex algebra structure. Explicitly we have $a_{(n)}=\frac{1}{(-n-1) !} T^{-n-1} a$.

(3) We have an obvious notion of morphisms of dg vertex Poisson algebras. We denote by dgVP the category of dg vertex Poisson algebras.

Similarly we have the notions of vertex Poisson superalgebra and vertex Poisson algebra (vpa for short).

Note that the translation $T$ of the commutative vertex algebra structure and that of the vertex Lie algebra structure are common.

We have notions of dg subalgebras, dg ideals, and tensor products of dg vpas. Details are omitted.

Since the structure maps $Y$ and $Y_{-}$of a dg vertex Poisson algebras are homogeneous with respect to the cohomological grading and the differential $d$ has the derivation property both for them, the proof of Lemma 4.1.22 works in the Poisson case, and we have:

Lemma. For a $\operatorname{dg}$ vpa $V$, the cohomology $H\left(V^{\bullet}, d\right)$ of the underlying complex has a structure of dg vertex Poisson algebra with trivial differential, called the cohomology vertex Poisson algebra.

We continue the observation in Remark 4.2.2 (2). For a $\operatorname{dg}$ vertex Poisson algebra $P=\left(P, d,|0\rangle, T, \cdot, Y_{-}\right)$, the quotient space $P / \operatorname{Im}(T)$ has a structure of dg Poisson algebra, of which the commutative multiplication and the Poisson bracket are given by

$$
\bar{a} \cdot \bar{b}:=\overline{a \cdot b}, \quad\{\bar{a}, \bar{b}\}:=\overline{a_{(0)} b}
$$

for $a, b \in P$. The Leibniz rule follows from (4.6). Since we have $[d, T]=0$, the quotient $P / \operatorname{Im}(T)$ inherits the dg structure $(P, d)$.

Definition 4.3.3. (1) We call the obtained $\operatorname{dg}$ Poisson algebra $P / \operatorname{Im}(T)$ the associated dg Poisson algebra of the dg vertex Poisson algebra $P$. We denote it by

$$
R_{P}^{\mathrm{co}}:=P / \operatorname{Im}(T) \text {. }
$$

(2) We denote the functor induced by the construction $P \mapsto R_{P}^{\text {co }}$ by

$$
R_{(-)}^{\mathrm{co}}: \mathrm{dgVP} \longrightarrow \mathrm{dgPA},
$$

where dgPA denotes the category of dg Poisson algebras.

We can immediately check that the functor $R_{(-)}^{\mathrm{co}}$ is a monoidal functor between the corresponding symmetric monoidal categories.

Remark. We refer Remark 4.4.6 (3) for the relation to Zhu's $C_{2}$-algebra $R_{V}$ of a vertex algebra $V$.

4.3.3. Vertex Poisson modules. We introduce the notion of modules over dg vertex Poisson algebras, following the non-dg version given in $[\mathrm{A} 12, \S 2.2]$ and $[\mathrm{A} 15, \S 2.2]$.

Definition 4.3.4. Let $P=\left(P, d,|0\rangle, T, \cdot, Y_{-}\right)$be a dg vertex Poisson algebra.

(1) A $d g$ vertex Poisson $P$-module is a complex $M=\left(M^{\bullet}, d_{M}\right)$ equipped with

- a structure $P \otimes M \rightarrow M, a \otimes m \mapsto a . m$ of dg module over the cdga $(P, \cdot,|0\rangle)$, and

- a morphism of complexes $P \otimes M \rightarrow M\left[z^{-1}\right] z^{-1}, a \otimes m \mapsto Y_{-}^{M}(a, z) m=\sum_{n \in \mathbb{N}}\left(a_{(n)}^{M} m\right) z^{-n-1}$ satisfying the following conditions for homogeneous $a, b \in P, m \in M$ and $l, n \in \mathbb{N}$.

(i) $Y_{-}^{M}(|0\rangle, z)=\mathrm{id}_{M}$. 
(ii) $(T a)_{(n)}^{M}=-n a_{(n-1)}^{M}$, where for $n=0$ we read the right hand side as 0 .

(iii) $\left[a_{(l)}^{M}, b_{(n)}^{M}\right]=\sum_{i \in \mathbb{N}}\left(\begin{array}{l}l \\ i\end{array}\right)\left(a_{(i)} b\right)_{(l+n-i)}^{M}$.

(iv) $a_{(n)}^{M}(b \cdot m)=\left(a_{(n)} b\right) \cdot m+(-1)^{|a||b|} b \cdot\left(a_{(n)}^{M} m\right)$.

(v) $(a \cdot b)_{(n)}^{M}=\sum_{l \in \mathbb{N}}\left(a_{(-l-1)} b_{(n+l)}^{M}+(-1)^{|a||b|} b_{(-l-1)} a_{(n+l)}^{M}\right)$.

(vi) $\left[d_{M}, Y_{-}^{M}(a, z)\right] m=Y_{-}^{M}(d a, z) m$.

(2) A morphism of dg vertex Poisson $P$-modules is naturally defined. We denote by $P$-dgVPMod the category of dg vertex Poisson $P$-modules, and by $P$-dgVPMod the corresponding $\infty$-category.

(3) A vertex Poisson $P$-module is a $\mathrm{dg}$ vertex Poisson $P$-module concentrating on the cohomological degree 0 . We denote by $P$-VPMod the category of vertex Poisson $P$-modules.

Now we give a vertex Poisson analogue of Fact 4.1 .8 on the equivalence of module categories. Let $P=$ $\left(P, d,|0\rangle, T, \cdot, Y_{-}\right)$be a dg vertex Poisson algebra. Recall the notation $a_{(n)}$ for an element $a \in P$ (Definition 4.3.2 (2)). We have the following line of constructions.

(1) We define the complex $U^{\prime}(P)$ to be the quotient of $P\left[t^{ \pm 1}\right]=P \otimes \mathbb{k}\left[t^{ \pm 1}\right]$ by the relation $(T a) \otimes t^{n}+$ $n a \otimes t^{n}=0$. We denote the image of $a \otimes t^{n}$ by $a_{[n]}$. We have $a_{[-n-1]}=\frac{1}{n !}\left(T^{n} a\right)_{[-1]}$ for $n \in \mathbb{N}$.

(2) We can define the binary operation $\cdot: U^{\prime}(P)^{\otimes 2} \rightarrow U^{\prime}(P)$ by

$$
a_{[-m-1]} \cdot b_{[-n-1]}:= \begin{cases}\left(\left(\frac{1}{m !} T^{m} a\right) \cdot\left(\frac{1}{n !} T^{n} b\right)\right)_{[-1]} & (m, n \in \mathbb{N}), \\ 0 & \text { (otherwise) } .\end{cases}
$$

Then $\left(U^{\prime}(P), \cdot\right)$ is a cdga with unit $|0\rangle_{[-1]}$.

(3) We can define the binary operation $\{-,-\}: U^{\prime}(P)^{\otimes 2} \rightarrow U^{\prime}(P)$ by

$$
\left\{a_{[m]}, b_{[n]}\right\}:= \begin{cases}\sum_{i \in \mathbb{N}}\left(\begin{array}{c}
m \\
i
\end{array}\right)\left(a_{(i)} b\right)_{[m+n-i]} & (m, n \in \mathbb{N}), \\
0 & \text { (otherwise). }\end{cases}
$$

Then $\left(U^{\prime}(P),\{-,-\}\right)$ is a dg Lie algebra by the proof of [FBZ, 4.1.2. Theorem].

(4) We can check that $\left(U^{\prime}(P), \cdot,\{-,-\}\right)$ is a dg Poisson algebra with unit $|0\rangle_{[-1]}$.

(5) We define $U^{\prime \prime}(P)$ to be the completion of $U^{\prime}(P)$ with respect to the $t$-adic topology. Thus we can regard it as a quotient of $P((t))=P \otimes \mathbb{k}((t))$. The completion $U^{\prime \prime}(P)$ inherits the $\mathrm{dg}$ Poisson algebra structure of $U^{\prime}(P)$. We further define $U(P):=\lim _{N} U^{\prime \prime}(P) / I_{N}$, where $I_{N}$ denotes the left Poisson ideal generated by $a_{[n]}(a \in P, n \in \mathbb{Z}, n>N)$. It also inherits the dg Poisson algebra structure.

(6) For $a \in P$, we denote $Y[a, z]:=\sum_{n \in \mathbb{Z}} a_{[n]} z^{-n-1}$. We define $\widetilde{U}(P)$ to be the quotient of $U(P)$ with respect to the relation $Y\left[a_{(-1)} b, z\right]=: Y[a, z] Y[b, z]:$, where the left hand side denotes the normal ordering (Definition 4.1.14).

(7) A dg Poisson $\widetilde{U}(P)$-module $M$ is called smooth if for any $m \in M$ and $a \in P$ we have $a_{[n]} \cdot m=0$ for $n \gg 0$.

Now we can apply the same argument in the proof of [FBZ, 5.1.6. Theorem], and we have;

Proposition 4.3.5. For a dg vertex Poisson algebra $P$, there is an equivalence of the category $P$-dgPMod of dg vertex Poisson $P$-modules and the category of smooth $\operatorname{dg}$ Poisson $\widetilde{U}(P)$-modules.

In the following, we will explain three constructions of dg vertex Poisson algebras.

(1) Symmetric algebra of a dg vertex Lie algebra (§4.3.4).

(2) Quasi-classical limit of a dg vertex algebra ( $\$ 4.3 .5)$.

(3) Level 0 vertex Poisson structure on the arc space of a dg Poisson algebra (§4.3.6).

(4) Associated graded space of Li filtration of a vertex algebra (§4.4).

4.3.4. Symmetric vertex Poisson algebra of vertex Li algebra. We first explain the symmetric algebra construction. See also [FBZ, 16.2.2] and [BD, 1.4.18].

Let $L=\left(L^{\bullet}, d, T_{L}, Y_{-}^{L}\right)$ be a dg vertex Lie algebra. Regarding $L$ as a complex, we denote by

$$
P:=\operatorname{Sym}(L)
$$

the symmetric algebra, which is a commutative dg algebra. Then $P$ has a structure $\left(|0\rangle, T, Y_{-}\right)$of dg vertex Poisson algebra where

- the vacuum $|0\rangle$ is the unit element of $P$,

- the translation $T$ is the extension of $T_{L}$ to $P$ by the Leibniz rule and the condition $T|0\rangle=0$. and

- the operation $Y_{-}$is uniquely determined by the condition that the injection $L \hookrightarrow P$ is a morphism of dg vertex Lie algebras and by the derivation condition. 
The following statement can be regarded as a vertex analogue of Remark 1.3.12 (3) on the momentum map. We omit the proof.

Lemma. Let $L$ be a dg vertex Lie algebra, $P$ be a dg vertex Poisson algebra, and $\mu: L \rightarrow P$ be a morphism of dg vertex Lie algebras. Then we have a unique morphism $\operatorname{Sym}(L) \rightarrow P$ of dg vertex Poisson algebras such that the restriction to $L$ coincides with $\mu$.

We have a twisted version which is similar as $\S 2.2(1)$. Let $L$ be a dg vertex Lie algebra, and assume that we are given a one-dimensional central extension $L^{b}$. In other words, we have a short exact sequence $0 \rightarrow \mathbb{k} \rightarrow L^{b} \rightarrow L \rightarrow 0$ of dg vertex Lie algebras, where $\mathbb{k}$ is the one-dimensional trivial vertex Lie algebra. Regarding $L^{b}$ as a complex, we define

$$
\operatorname{Sym}^{b}(L):=\operatorname{Sym}\left(L^{b}\right) / I
$$

to be the quotient of the symmetric algebra $\operatorname{Sym}\left(L^{b}\right)$ by the ideal $I$ generated by the difference of embeddings $\mathbb{k}=\operatorname{Sym}\left(L^{b}\right)^{0} \hookrightarrow \operatorname{Sym}\left(L^{b}\right)$ and $\mathbb{k} \hookrightarrow \mathfrak{l}^{\mathfrak{b}}=\operatorname{Sym}\left(L^{b}\right)^{1} \hookrightarrow \operatorname{Sym}\left(L^{b}\right)$. It inherits the dg vertex Poisson structure from $\operatorname{Sym}\left(L^{b}\right)$.

For later reference, we name the obtained dg vertex Lie algebras as:

Definition 4.3.6. Let $L$ be a dg vertex Lie algebra.

(1) W call the dg vertex Poisson algebra $\operatorname{Sym}(L)$ the symmetric $d g$ vpa.

(2) Assume that we are given a one-dimensional central extension $L^{\mathrm{b}}$ of $L$. We call the dg vertex Poisson algebra $\operatorname{Sym}^{b}(L)$ the twisted symmetric dg vpa.

4.3.5. Quasi-classical limit construction. Next we turn to the second construction by a quasi-classical limit of a vertex superalgebra. See [FBZ, 16.2.3-7] for the detail. Here we only explain a particular case.

Let $U$ be a complex, and consider the free fermionic vertex algebra $\bigwedge^{\frac{\infty}{2}}(U)$ in Definition 4.1.25. We use the linear basis $\left\{\psi_{i} \mid i \in I\right\}$ of $U$ and the dual basis $\left\{\psi_{j}^{*} \mid j \in I\right\}$ of $U^{*}$ therein. In particular, $\bigwedge^{\frac{\infty}{2}}(U)$ has a basis consisting of PBW monomials (4.2).

We define $\Lambda_{\hbar}$ to be the $\mathbb{k}\left[\hbar^{-1}\right]$-lattice spanned by the rescaled monomials

$$
\hbar^{-k}\left(\psi_{i_{1}} t^{m_{1}}\right) \cdots\left(\psi_{i_{k}} t^{m_{k}}\right)\left(\psi_{j_{1}}^{*} t^{n_{1}-1} d t\right) \cdots\left(\psi_{j_{l}}^{*} t^{n_{l}-1} d t\right)|0\rangle \quad\left(m_{1}<\cdots<m_{k}<0, n_{1}<\cdots<n_{l} \leq 0\right) .
$$

In other words, we shift $\psi_{i} \mapsto \hbar^{-1} \psi_{i}$ and preserve $\psi_{j}^{*}$. Then we set

$$
\bar{\bigwedge}^{\frac{\infty}{2}}(U):=\bigwedge_{\hbar} / \hbar^{-1} \bigwedge_{\hbar}
$$

The image of the generators are denoted by $\bar{\psi}_{i} t^{m}$ and $\bar{\psi}_{j}^{*} t^{n-1} d t$. It inherits the left dg Cl-module structure of $\bigwedge^{\frac{\infty}{2}}(U)$, and has an action of the dg subalgebra of $\mathrm{Cl}\left[\hbar^{-1}\right]$ generated by

$$
\bar{\psi}_{i} t^{m}:=\hbar \psi_{i} t^{m}, \quad \bar{\psi}_{j}^{*} t^{n-1} d t:=\psi_{j}^{*} t^{n-1} d t .
$$

The commutation relations of these generators are

$$
\left[\bar{\psi}_{i} t^{m}, \bar{\psi}_{j}^{*} t^{n-1} d t\right]=\delta_{i, j} \delta_{m+n, 0}, \quad\left[\bar{\psi}_{i} t^{m}, \bar{\psi}_{j} t^{n}\right]=0=\left[\bar{\psi}_{i}^{*} t^{m-1} d t, \bar{\psi}_{j}^{*} t^{n-1} d t\right] .
$$

Thus the complex $\bar{\Lambda}^{\frac{\infty}{2}}(U)$ has a linear basis consisting of the elements of the form

$$
\left(\bar{\psi}_{i_{1}} t^{m_{1}}\right) \cdots\left(\bar{\psi}_{i_{k}} t^{m_{k}}\right)\left(\bar{\psi}_{j_{1}}^{*} t^{n_{1}-1} d t\right) \cdots\left(\bar{\psi}_{j_{l}}^{*} t^{n_{l}-1} d t\right)|0\rangle \quad\left(m_{1}<\cdots<m_{k}<0, n_{1}<\cdots<n_{l} \leq 0\right) .
$$

The complex $\bar{\Lambda}^{\frac{\infty}{2}}(U)$ has a structure of dg vertex Poisson algebra induced by the dg vertex algebra structure on $\bigwedge^{\frac{\infty}{2}}(U)$. As a commutative dg algebra, it is isomorphic to $\operatorname{Sym}\left(U((t))[1] \oplus U^{*}((t))[-1] d t\right)$, and the differential is given by $\partial_{t}$. The vertex Lie algebra structure $Y_{-}$is determined by

$$
\begin{aligned}
& Y_{-}\left(\bar{\psi}_{i} t^{-1}|0\rangle, z\right)=\bar{\psi}_{i}(z):=\sum_{l \in \mathbb{N}}\left(\bar{\psi}_{i} t^{l}\right) z^{-l-1}, \quad Y_{-}\left(\bar{\psi}_{j}^{*} t^{-1} d t|0\rangle, z\right)=\bar{\psi}_{j}^{*}(z):=\sum_{l \in \mathbb{N}}\left(\bar{\psi}_{j}^{*} t^{l}\right) z^{-l-1}, \\
& Y_{-}\left(\bar{\psi}_{i} t^{m}|0\rangle, z\right)=\frac{1}{(-m-1) !} \partial_{z}^{-m-1} \bar{\psi}_{i}(z), \quad Y_{-}\left(\bar{\psi}_{j}^{*} t^{n-1} d t|0\rangle, z\right)=\frac{1}{(-n) !} \partial_{z}^{-n} \bar{\psi}_{i}^{*}(z) .
\end{aligned}
$$

It also inherits the charge grading $\bigwedge^{\frac{\infty}{2}+\bullet}(U)$ given by charge $\left(\overline{\psi_{i}} t^{m}\right)=-1$, charge $\left(\overline{\psi_{i}} t^{n-1} d t\right)=1$. We denote $\bar{\Lambda}^{\frac{\infty}{2}}(U)$ to emphasize this charge grading.

Definition 4.3.7. We call the obtained dg vertex Poisson algebra ${\bigwedge^{\frac{\infty}{2}}}^{(U)}$ the free fermionic vertex Poisson algebra. 
We can check that the associated dg Poisson algebra (Definition 4.3.3) is the classical Clifford algebra $\overline{\mathrm{Cl}}(U)$ (Definition 2.2.1).

4.3.6. Vertex Poisson structure of arc space. We cite from $[\mathrm{A} 12, \S 2.3]$ the third construction of a dg vertex Poisson algebra via the arc space of a dg Poisson algebra. Recall the arc space $J_{\infty}(R)$ of a commutative dg algebra $R$ (Definition 3.2.5). We denote by $T$ the 0 -derivation on $J_{\infty}(R)$.

Proposition 4.3.8. For a dg Poisson algebra $R$ which is of finite type as a commutative algebra, there is a unique dg vertex Poisson algebra structure on $J_{\infty}(R)$ such that

$$
u_{(n)}\left(T^{l} v\right)= \begin{cases}\frac{l !}{(l-n) !} T^{l-n}\{u, v\}_{R} & (l \geq n), \\ 0 & (l<n) .\end{cases}
$$

for $u, v \in R \subset J_{\infty}(R)$ and $l \in \mathbb{N}$. It is called the level $0 d g$ vertex Poisson algebra.

Proof. We follow the proof of the non-dg case given in [A12, Proposition 2.3.1]. By the formula (4.7), we have a well-defined morphism of complexes

$$
R \longrightarrow \operatorname{Der}\left(J_{\infty}(R)\right)\left[\left[z^{-1}\right]\right] z^{-1}, \quad u \longmapsto u_{-}(z):=\sum_{n \in \mathbb{N}} u_{(n)} z^{-n-1} .
$$

We obviously have $u_{-}(z) \in \operatorname{Der}\left(J_{\infty}(R)\right)^{|u|}\left[\left[z^{-1}\right]\right] z^{-1}$ for homogeneous $u \in R$, and can check the conditions (i)-(iv) in Definition 4.2.1 of dg vertex Lie algebra structure (see Proof of Lemma 4.2.5). This morphism is extended to $Y_{-}(-, z): J_{\infty}(R) \rightarrow \operatorname{Der}\left(J_{\infty}(R)\right)\left[\left[z^{-1}\right]\right] z^{-1}$ by

$$
Y_{-}(a, z)\left(T^{l} u\right):=\left(e^{z T}\left(-\partial_{z}\right)^{l} u_{-}(-z) a\right)_{-}
$$

for $a \in J_{\infty}(R), u \in R$ and $l \in \mathbb{N}$. By [A12, Proposition 2.3.1], we know that it defines a vertex Poisson superalgebra structure on the associated commutative superalgebra $J_{\infty}(R)^{\text {even }} \oplus J_{\infty}(R)^{\text {odd }}$. Thus it remains to check the condition (iv) in Definition 4.2 .1 and the condition $Y_{-}(a, z) \in \operatorname{Der}\left(J_{\infty}(R)\right)^{|a|}\left[\left[z^{-1}\right]\right] z^{-1}$ for homogeneous $a \in J_{\infty}(R)$, which are obvious.

Hereafter we regard $J_{\infty}(R)$ as the level 0 vertex Poisson algebra unless otherwise stated. Recall the associated dg Poisson algebra $R_{P}^{\text {co }}$ for a dg vertex Poisson algebra $P$ (Definition 4.3.3). By the construction, we have:

Lemma 4.3.9. For a commutative dg algebra $R$ of finite type, we have

$$
R_{J_{\infty}(R)}^{\text {co }} \simeq R \text {. }
$$

Example 4.3.10. Let $\mathfrak{l}$ be a dg Lie algebra and consider the Kostant-Kirillov dg Poisson algebra $R=\operatorname{Sym}(\mathfrak{l})$ (Notation 1.3.10). Then we have the level 0 vertex Poisson superalgebra $J_{\infty}(\operatorname{Sym}(\mathfrak{l}))$.

By Remark 3.1.4 of the description of $J_{\infty}(\operatorname{Sym}(\mathfrak{l}))$, we have

$$
J_{\infty}(\operatorname{Sym}(\mathfrak{l})) \simeq \operatorname{Sym}(\mathfrak{l}[[t]])
$$

as commutative $d g$ algebras, where $\mathfrak{l}[[t]]=\mathfrak{l} \otimes \mathbb{k}[[t]]$ is the tensor product in $\mathrm{dgVec}$. Let us denote an element of $\mathfrak{l}[[t]]$ by $x f:=x \otimes f$ with $x \in \mathfrak{l}$ and $f=f(t) \in \mathbb{k}[[t]]$. Then the level 0 dg vertex Poisson structure is given by

$$
(x f)_{(n)}(y g)=\delta_{n, 0}[x, y]_{\mathfrak{r}} \otimes(f g) .
$$

This is nothing but the standard Lie algebra structure on $\mathfrak{l}[[t]]$. We can also check that the vertex Lie algebra structure on the restriction $\mathfrak{l}[[t]]=\operatorname{Sym}(\mathfrak{l}[[t]])^{1} \hookrightarrow J_{\infty}(\operatorname{Sym}(\mathfrak{l}))$ coincides with the level 0 vertex Lie algebra $J_{\infty}(\mathfrak{l})$ in Lemma 4.2.5 (compare (4.4) and (4.7)). Thus we have an isomorphism of dg vertex Poisson algebras

$$
J_{\infty}(\operatorname{Sym}(\mathfrak{l})) \simeq \operatorname{Sym}\left(J_{\infty}(\mathfrak{l})\right)
$$

where the right hand side denotes the symmetric vertex Poisson algebra (Definition 4.3.6).

Recall the twisted symmetric vertex Poisson algebra $\operatorname{Sym}^{b}(L)$ for a dg vertex Poisson algebra (Definition 4.3.6). Using this construction, we can recover the free fermion vertex Poisson algebra $\bigwedge^{\frac{\infty}{2}}(U)$ in Definition 4.3.7. The present construction is in fact a coordinate-dependent version of the Clifford coisson algebra in [BD, 1.4.21].

Let $U$ be a complex. We denote by $U^{*}=\underline{\operatorname{Hom}}(U, \mathbb{k})$ the dual complex, and by $\langle\cdot, \cdot\rangle: U^{*} \otimes U \rightarrow \mathbb{k}$ the natural pairing. We also denote $J_{\infty}(U):=U[[t]]$ and consider it as a $\mathrm{dg}$ vertex Lie algebra attached to the trivial dg Lie algebra $U$. (Lemma 4.2.5). Similarly we have a dg vertex Lie algebra $J_{\infty}(U)^{*}=$ $U^{*}\left[\left[t^{-1}\right]\right]$, and the direct sum $J_{\infty}(U)[1] \oplus J_{\infty}(U)^{*}[-1]$. By the pairing $\langle\cdot, \cdot\rangle$, we have a one-dimensional 
central extension of the direct sum, and thus we have the twisted symmetric dg vertex Poisson algebra $\operatorname{Sym}^{b}\left(J_{\infty}(U)[1] \oplus J_{\infty}(U)^{*}[-1]\right)$.

Definition 4.3.11. For a complex $U$, we denote the above dg vertex Poisson algebra by

$$
\mathrm{Cl}_{\mathrm{co}}\left(J_{\infty}(U)\right):=\operatorname{Sym}^{\mathrm{b}}\left(J_{\infty}(U)[1] \oplus J_{\infty}(U)^{*}[-1]\right)
$$

and call it the Clifford vertex Poisson algebra.

By comparing the description in $\S 4.3 .5$, we have

$$
\mathrm{Cl}_{\mathrm{co}}\left(J_{\infty}(U)\right) \simeq \bar{\Lambda}^{\frac{\infty}{2}}(U)
$$

as dg vertex Poisson algebras. In particular, we have:

Corollary 4.3.12. The associated dg Poisson algebra (Definition 4.3.3) of $\mathrm{Cl}_{\mathrm{co}}\left(J_{\infty}(U)\right)$ coincides with the classical Clifford algebra $\overline{\mathrm{Cl}}(U)$ :

$$
R_{\mathrm{Cl}}^{\mathrm{co}\left(J_{\infty}(U)\right)} \simeq \overline{\mathrm{Cl}}(U)
$$

Using $\mathrm{Cl}_{\mathrm{co}}\left(J_{\infty}(U)\right)$ we will introduce coisson BRST reduction in the following $\S 5$.

Remark 4.3.13. In some literature such as [FBZ, §16.7], what we call coisson BRST reduction is called the classical BRST reduction. In that terminology $\mathrm{Cl}_{\mathrm{co}}\left(J_{\infty}(U)\right)$ should be called the classical Clifford algebra, conflicting our terminology (Definition 2.2.1).

4.4. Li filtration. In this subsection we introduce the Li filtration for a dg vertex algebra. Main references are [Li05] and [A15].

4.4.1. Definition. In the following we use:

Notation. For a linear space $V$ and a subset $S \subset V$, we denoted by $\langle S\rangle_{\operatorname{lin}}$ the linear subspace spanned by the elements in $S$. In the case $S=\left\{s_{i} \mid i \in I\right\}$, we also denote it by $\left\langle s_{i} \mid i \in I\right\rangle_{\operatorname{lin}}$.

For a dg vertex algebra $V, a \in V$, and a $\operatorname{dg} V$-module $M$, we denote $Y_{M}(a, z)=\sum_{n \in \mathbb{Z}} a_{(n)}^{M} z^{-n-1}$ as before. Then we define a linear subspace $F^{p} M \subset M$ by

$$
F^{p} M:=\left\langle\left(a_{1}^{M}\right)_{\left(-n_{1}-1\right)} \cdots\left(a_{r}^{M}\right)_{\left(-n_{r}-1\right)} m \mid m \in M, r \in \mathbb{Z}_{>0}, a_{i} \in V, n_{i} \in \mathbb{N}, \sum_{i=1}^{r} n_{i} \geq p\right\rangle_{\operatorname{lin}} .
$$

Then we have a decreasing filtration $M=F^{0} M \supset F^{1} M \supset F^{2} M \supset \cdots$ of linear spaces. By induction using $a_{(n)} M^{i} \subset M^{i+j}$ and $d_{M}\left(a_{(n)}^{M} m\right)=\left(d_{V} a\right)_{(n)} m+(-1)^{|a|} a_{(n)}\left(d_{M} m\right)$ in Definition 4.1.21 of $\mathrm{dg} V$-module, we can check that the differential $d_{M}$ preserves the Li filtration: $d_{M}\left(F^{p} M\right) \subset F^{p} M$ for any $p \in \mathbb{Z}$. Thus $F^{\bullet} M$ is in fact a filtration of complexes.

Definition 4.4.1 ([Li05, Definition 2.7, Lemma 2.8]). For a dg vertex algebra $V$ and a $\operatorname{dg} V$-module $M$, we call the decreasing filtration of complexes

$$
M=F^{0} M \supset F^{1} M \supset F^{2} M \supset \cdots
$$

the Li filtration of $M$. In particular, we can take $M:=V$ and have the Li filtration of $V$ :

$$
V=F^{0} V \supset F^{1} V \supset F^{2} V \supset \cdots .
$$

Below we set $F^{p} M:=M$ for $p \in \mathbb{Z}_{<0}$.

Lemma 4.4.2 ([Li05, Lemma 2.9, Proposition 2.11]). Let $V$ and $M$ be as in Definition 4.4.1.

(1) For any $p \in \mathbb{Z}_{\geq 1}$, we have

$$
F^{p} M=\left\langle a_{(-i-1)} m \mid a \in V, i \in \mathbb{Z}, 1 \leq i \leq p, m \in F^{p-i} M\right\rangle_{\operatorname{lin}} .
$$

In particular, taking $M:=V$, we have

$$
F^{1} V=C_{2}(V):=\left\langle a_{(-2)} b \mid a, b \in V\right\rangle_{\text {lin }} .
$$

(2) $a_{(n)} F^{q} M \subset F^{p+q-n-1} M$ for any $p, q \in \mathbb{Z}, a \in F^{p} V$ and $n \in \mathbb{Z}$.

(3) $a_{(n)} F^{q} M \subset F^{p+q-n} M$ for any $p, q \in \mathbb{Z}, a \in F^{p} V$ and $n \in \mathbb{N}$.

(4) $T\left(F^{p} V\right) \subset F^{p+1} V$ for any $p \in \mathbb{Z}$.

By these properties of the Li filtration, we have a canonical construction of vertex Poisson algebra from any vertex algebra. We follow [A15] for the notation.

Fact 4.4.3 ([Li05, Theorem 2.12]). Let $V=(V, d,|0\rangle, T, Y)$ be a dg vertex algebra. 
(1) The associated graded space

$$
\operatorname{gr}^{F} V:=\bigoplus_{p \in \mathbb{N}} F^{p} V / F^{p+1} V
$$

is a commutative dg algebra equipped with an additional 0-derivation $\delta$ given by

$$
\sigma_{p}(a) \cdot \sigma_{q}(b):=\sigma_{p+q}\left(a_{(-1)} b\right), \quad \delta \sigma_{p}(a):=\sigma_{p+1}\left(a_{(-2)}|0\rangle\right)=\sigma_{p+1}(T(a))
$$

Here $\sigma_{p}: F^{p} V \rightarrow F^{p} V / F^{p+1} V$ denotes the projection.

(2) $\operatorname{gr}^{F} V$ has a structure of dg vertex Poisson algebra whose commutative dg vertex algebra structure is given by (1) and Lemma 4.3.1, and whose dg vertex Lie algebra structure is given by

$$
\sigma_{p}(a)_{(n)} \sigma_{q}(b):=\sigma_{p+q-n}\left(a_{(n)} b\right) \quad(n \in \mathbb{N}) .
$$

Here we understand $\sigma_{r}(a)=0$ for $r<0$.

Precisely speaking, the statement in [Li05, Theorem 2.12] is for the non-dg case, but the same proof works in the dg case.

For a morphism $\phi: V \rightarrow W$ of dg vertex algebras, we have $\phi\left(F^{p} V\right) \subset F^{p} W$, which induces a morphism $\operatorname{gr}^{F} V \rightarrow \operatorname{gr}^{F} W$ of dg vertex Poisson algebras. Thus we obtain:

Lemma 4.4.4. Taking the associated graded space of the Li filtration, we have a functor

$$
\operatorname{gr}^{F}: \mathrm{dgVA} \longrightarrow \mathrm{dgVP} \text {. }
$$

It is a monoidal functor of the symmetric monoidal structures.

As a corollary of Fact 4.4.3, we have a dg Poisson algebra on the quotient $F^{0} V / F^{1} V$. Following the terminology in $[\mathrm{A} 12, \mathrm{~A}]$, we give:

Definition 4.4.5. Let $V$ be a dg vertex algebra.

(1) The complex

$$
R_{V}:=F^{0} V / F^{1} V=V / C_{2}(V) \subset \operatorname{gr}^{F} V
$$

has a structure of dg Poisson algebra whose multiplication $\cdot$ and Poisson bracket $\{-,-\}$ are given by

$$
\bar{a} \cdot \bar{b}:=\overline{a_{(-1)} b}, \quad\{\bar{a}, \bar{b}\}:=\overline{a_{(0)} b} .
$$

Here $\bar{a}:=\sigma_{0}(a)$ denotes the image of $a \in V$ in $R_{V}$. The resulting dg Poisson algebra $R_{V}$ is called Zhu's $C_{2}$-algebra of $V$.

(2) We denote the functor induced by the construction $V \mapsto R_{V}$ by

$$
R_{(-)}: \mathrm{dgVP} \longrightarrow \mathrm{dgPA},
$$

where dgPA denotes the category of dg Poisson algebras.

(3) If $R_{V}$ is concentrated in negative cohomological degrees, then we denote the corresponding affine derived Poisson scheme by

$$
X_{V}:=\operatorname{Spec}\left(R_{V}\right)
$$

and call it the associated derived scheme of $V$.

If $V$ is a plain vertex algebra, i.e., concentrated in cohomological degree 0 , then $R_{V}$ is a Poisson algebra, and we call the corresponding affine Poisson scheme $X_{V}$ the associated scheme, which recovers the terminology in $[\mathrm{A} 15, \mathrm{~A}]$.

Remark 4.4.6. (1) In the non-dg case, the Poisson algebra $R_{V}$ was introduced by Y. Zhu to give a nice finiteness condition on vertex operator algebras [Z96, §4.4].

(2) We can confirm the Poisson structure directly by using Remark 4.1.6 (1).

(3) We can easily find that Zhu's $C_{2}$-algebra $R_{V}$ coincides with the associated dg Poisson algebra of $\operatorname{gr}^{F} V$ (Definition 4.3.3):

$$
R_{V} \simeq R_{\mathrm{gr}^{F} V}^{\mathrm{co}}
$$

Example 4.4.7 ([A15, Lemma 4.5]). Let us consider the free fermionic vertex algebra $\bigwedge^{\frac{\infty}{2}}(U)$ (Definition 4.1.25) for a complex $U$. Using the commutation relation (4.1) and the linear basis (4.2), we can check

$$
\operatorname{gr}^{F} \bigwedge^{\frac{\infty}{2}}(U) \simeq \bar{\Lambda}^{\frac{\infty}{2}}(U)
$$


as dg vertex Poisson algebras, where the right hand side denotes the free fermionic vertex Poisson algebra (Definition 4.3.7). In particular, on Zhu's $C_{2}$-algebras we have

$$
R_{\wedge \infty / 2(U)} \simeq \overline{\mathrm{Cl}}(U)
$$

as dg Poisson algebras, where $\overline{\mathrm{Cl}}(U)$ denotes the classical Clifford algebra (Definition 2.2.1).

For later use, let us recall:

Definition 4.4.8. A vsa $V$ is separated if $\bigcap_{n \in \mathbb{N}} F^{n} V=0$.

By [Li05, Proposition 3.9], if a vertex algebra $V$ has a lower truncated $\mathbb{Z}$-gradation (i.e., there is some $n \in \mathbb{Z}$ such that $V$ is $\mathbb{Z}_{\geq n}$-graded), then it is separated.

4.4.2. The case of universal affine vertex algebra. Let us explain the notions introduced so far in the case of the universal affine vertex algebra (\$4.1.4).

Recall Notation 4.1.12 and Definition 4.1.13. In particular

- $\mathfrak{g}$ is the Lie algebra of the semi-simple algebraic group over $\mathbb{C}$, and

- $\widehat{\mathfrak{g}}=\mathfrak{g}((t)) \oplus \mathbb{C} K$ is the the derived algebra of the non-twisted affine Lie algebra associated to $\mathfrak{g}$.

- $V_{k}(\mathfrak{g})$ is the universal affine vertex algebra at level $k \in \mathbb{C}$.

Let $\left\{x_{i} \mid i=1, \ldots, \operatorname{dim} \mathfrak{g}\right\}$ be a linear basis of $\mathfrak{g}$. Then we have a PBW basis of $V_{k}(\mathfrak{g})($ Fact 4.1 .15$):$ It consists of monomials of the form $\left(x_{i_{1}} t^{n_{1}}\right) \cdots\left(x_{i_{l}} t^{n_{l}}\right)|0\rangle$, where $n_{1} \leq \cdots \leq n_{l}<0$ and if $n_{j}=n_{j+1}$ then $i_{j} \leq i_{j+1}$.

Recall Definition 4.4.1 of the Li filtration $F^{\bullet} V_{k}(\mathfrak{g})$. By the remark after Definition 4.4.8, we find that $F^{\bullet} V_{k}(\mathfrak{g})$ is separated. Also recall Zhu's $C_{2}$-algebra $R_{V_{k}(\mathfrak{g})}:=V_{k}(\mathfrak{g}) / F^{1} V_{k}(\mathfrak{g})$ (Definition 4.4.5). By the PBW basis above we find that the set $\left\{x_{i} t^{n} \mid i=1, \ldots, \operatorname{dim} \mathfrak{g}, n \in \mathbb{Z}_{<0}\right\}$ generates $R_{V_{k}(\mathfrak{g})}$ as a commutative algebra, and that we have $F^{1} V_{k}(\mathfrak{g})=\mathfrak{g}\left[t^{-1}\right] t^{-2} V_{k}(\mathfrak{g})$. Then we further find that there is an isomorphism

$$
\operatorname{Sym}(\mathfrak{g}) \stackrel{\sim}{\longrightarrow} R_{V_{k}(\mathfrak{g})}=V_{k}(\mathfrak{g}) / F^{1} V_{k}(\mathfrak{g}), \quad x \longmapsto \overline{\left(x t^{-1}\right)|0\rangle}
$$

of commutative algebras. By checking the Poisson brackets on both sides, we have (1) in the following fact.

Fact 4.4.9 ([A12, Proposition 2.7.1]). (1) The map (4.8) gives the following isomorphism of Poisson algebras.

$$
\operatorname{Sym}(\mathfrak{g}) \stackrel{\sim}{\longrightarrow} R_{V_{k}(\mathfrak{g})} .
$$

(2) The same map induces an isomorphism

$$
J_{\infty}(\operatorname{Sym}(\mathfrak{g})) \stackrel{\sim}{\longrightarrow} \operatorname{gr}^{F} V_{k}(\mathfrak{g})
$$

of vertex Poisson algebras, where the left hand side denotes the level 0 vertex algebra (Fact 4.3.8) associated to the Kirillov-Kostant Poisson algebra $\operatorname{Sym}(\mathfrak{g})$.

In particular, both $R_{V_{k}(\mathfrak{g})}$ and $\operatorname{gr}^{F} V_{k}(\mathfrak{g})$ are independent of the level $k$. See [A12, Proposition 2.7.1] for a proof of this fact.

Finally we consider the module category of $J_{\infty}(\operatorname{Sym}(\mathfrak{g}))$. By Proposition 4.3.5 a vertex Poisson module is equivalent to a smooth Poisson module of the associated Poisson algebra. By the construction in $\S 4.3 .3$, we can check:

Lemma. As Poisson algebras we have $\widetilde{U}\left(J_{\infty}(\operatorname{Sym}(\mathfrak{g}))\right) \simeq \operatorname{Sym}(\mathfrak{g}[[t]])$, where the latter denotes the KirillovKostant Poisson algebra for the Lie algebra $\mathfrak{g}[[t]]$.

Then by the definition of smooth Poisson modules $(\S 4.3 .3(7))$, we can restate Proposition 4.3.5 as:

Proposition 4.4.10. A vertex Poisson module over $J_{\infty}(\operatorname{Sym}(\mathfrak{g}))$ is equivalent to a smooth representation of the Lie algebra $\mathfrak{g}[[t]]$, i.e., a representation $M$ such that $\left(x t^{n}\right) \cdot m=0$ for any $x \in \mathfrak{g}, m \in M$ and $n \gg 0$.

\section{Coisson BRSt REduction And GLuing Procedure For ARC spaces}

In this section we introduce the BRST reduction for dg vertex Poisson algebras and a dg vertex Poisson analogue of the category MT. 
5.1. Coisson BRST complex. In this subsection we introduce the BRST complex for dg vertex Poisson algebras. The construction is a natural analogue of classical BRST complex in $\S 2.2$. We work over a field $\mathbb{k}$ of characteristic 0 in this subsection.

Remark 5.1.1. Our argument is in fact a coordinate-dependent version of the arguments in [BD, 1.4.21-26], where the BRST complex is introduced for coisson algebras. Let us give a brief comment on the theory of coisson algebras. See [BD, 0.15, 2.6, 3.8.6] and [FG12] for the detail, and also [FBZ, Chap. 19] for a related exposition.

Let $X$ be a smooth algebraic variety. A right D-module $\mathcal{M}$ on the Ran space $\operatorname{Ran}(X)$ of $X$ is a family of right D-modules $\mathcal{M}_{X^{I}} \in \operatorname{DMod}\left(X^{I}\right)$ for finite sets $I$ satisfying a compatibility condition for every surjection $I \rightarrow J$. We have a natural fully faithful embedding $\iota: \operatorname{DMod}(X) \rightarrow \operatorname{DMod}(\operatorname{Ran}(X))$ of the category of right D-modules on $X$ into that on $\operatorname{Ran}(X)$. On the category $\operatorname{DMod}(\operatorname{Ran}(X))$, we can also construct two structures of symmetric monoidal categories denoted by $\otimes^{!}$and $\otimes^{\star}$. The monoidal structure $\otimes^{!}$is equivalent to the standard one on left D-modules on $X$, and the other structure $\otimes^{*}$ is designed to reflect operator product expansion nicely. A Lie algebra object in the monoidal category $\operatorname{DMod}(\operatorname{Ran}(X))^{\otimes^{*}}$ which lies in the essential image of $\iota$ is called a $\star$-Lie algebra on $X$. Similarly, we have the notion of !-commutative algebra on $X$. Then a coisson algebra over $X$ is a "compound Poisson algebra object" in $\operatorname{DMod}(\operatorname{Ran}(X))$, i.e., a combination of !-commutative ring structure and $\star$-Lie algebra structure.

A vertex Lie algebra is equivalent to a $\star$-Lie algebra on $X=\mathbb{A}^{1}$ which is equivariant under the action of affine transformations of $\mathbb{A}^{1}$. Similarly, a vertex Poisson algebra is equivalent to an equivariant coisson algebra on $\mathbb{A}^{1}$. Below we introduce the BRST complex for vertex Poisson algebras by replacing "Lie algebra" in the argument on classical BRST complex with "ᄎ-Lie algebra", or "vertex Lie algebra".

Recall that for a dg Lie algebra $\mathfrak{l}$, we have a contractible dg Lie algebra $\mathfrak{l}_{\dagger}(\S 1.2 .4)$. By the same way, for a dg vertex Lie algebra $L$, we have a dg vertex Lie algebra structure on the contractible complex Cone $\left(\operatorname{id}_{L}\right)=L \oplus L[1]$, which will be denoted by $L_{\dagger}$.

Let $\mathfrak{l}$ be a dg Lie algebra, and $L:=J_{\infty}(\mathfrak{l})$ be the level 0 vertex Lie algebra (Lemma 4.2.5). As a complex we have $L \simeq \mathfrak{l}[[t]]$. Recall also the Clifford vertex Poisson algebra $\operatorname{Cl}_{\mathrm{co}}\left(J_{\infty}(\mathfrak{l})\right)=\operatorname{Sym}^{\mathfrak{b}}\left(L[1] \oplus L^{*}[-1]\right)$ in Definition 4.3.11,

Using these notations, we have the following vertex Poisson analogue of Lemma 2.2.2. We omit the proof.

Lemma. Let $\mathfrak{l}$ be a dg Lie algebra, $L:=J_{\infty}(\mathfrak{l}), P$ be a dg vertex Poisson algebra, and $\mu_{\mathrm{co}}: L \rightarrow P$ be a morphism of dg vertex Lie algebras.

(1) The adjoint action of $L:=J_{\infty}(\mathfrak{l})=\mathfrak{l}[[t]]$ on itself as a $\operatorname{dg}$ Lie algebra yields a morphism $\beta: L \rightarrow$ $\mathrm{Cl}_{\mathrm{co}}(L)$ of dg vertex Lie algebras as a composition

$$
\beta: L \longrightarrow L \otimes L^{*} \stackrel{\sim}{\longrightarrow} L[1] \otimes L^{*}[-1] \hookrightarrow \mathrm{Cl}_{\mathrm{co}}(L)^{0} .
$$

(2) Let

$$
\ell: L_{\dagger} \longrightarrow \mathrm{Cl}_{\mathrm{co}}(L) \otimes P
$$

be the morphism of complex given by

$$
\ell^{0}:=1 \otimes \mu_{\mathrm{co}}+\beta \otimes 1: L \longrightarrow \mathrm{Cl}_{\mathrm{co}}(L)^{0} \otimes P, \quad \ell^{-1}: L[1] \longrightarrow \mathrm{Cl}_{\mathrm{co}}(L)^{-1} \longrightarrow \mathrm{Cl}_{\mathrm{co}}(L) \otimes P .
$$

Then $\ell$ is a morphism of dg vertex Lie algebras.

(3) We define the following elements.

- $\widetilde{\mu}_{\mathrm{co}} \in L^{*} \otimes P \subset\left(\mathrm{Cl}_{\mathrm{co}}(L) \otimes P\right)^{1}$ is the element corresponding to $\mu_{\mathrm{co}}$.

- $\beta^{\prime} \in L^{*} \otimes \mathrm{Cl}_{\mathrm{co}}(L)^{0}$ is the element corresponding to $\beta$.

- $\beta^{\prime \prime} \in \mathrm{Cl}_{\mathrm{co}}(L)^{1}$ is the image of $\beta^{\prime}$ by the product map $L^{*}[-1] \otimes \mathrm{Cl}_{\mathrm{co}}(L) \rightarrow \mathrm{Cl}_{\mathrm{co}}(L)$.

- $\widetilde{\beta} \in\left(\mathrm{Cl}_{\mathrm{co}}(L) \otimes P\right)^{1}$ is the image of $\beta^{\prime \prime}$ by $\mathrm{Cl}_{\mathrm{co}}(L) \rightarrow \mathrm{Cl}_{\mathrm{co}}(L) \otimes P$.

Then the coisson BRST charge

$$
Q_{\mathrm{co}}:=\widetilde{\mu}_{\mathrm{co}}+\frac{1}{2} \widetilde{\beta} \in\left(\mathrm{Cl}_{\mathrm{co}}(L) \otimes P\right)^{1}
$$

satisfies $\left(Q_{\text {co }}\right)_{(0)}{ }^{2}=0$, where $Y_{-}\left(Q_{\text {co }}, z\right)=\sum_{n \in \mathbb{N}}\left(Q_{\text {co }}\right)_{(n)} z^{-n-1}$ denotes the vertex Lie algebra structure of $\mathrm{Cl}_{\mathrm{co}}(L) \otimes P$.

Now we can introduce:

Definition 5.1.2. Let $\mathfrak{l}$ be a dg Lie algebra, and $P$ be a dg vertex Poisson algebra.

(1) We call a morphism $\mu_{\mathrm{co}}: J_{\infty}(\mathfrak{l}) \rightarrow P$ of dg vertex Lie algebras a coisson momentum map. 
(2) Given a coisson momentum map $\mu_{\mathrm{co}}: J_{\infty}(\mathfrak{l}) \rightarrow P$, we define the coisson BRST complex to be the dg vertex Poisson algebra

$$
\operatorname{BRST}_{\mathrm{co}}\left(J_{\infty}(\mathfrak{l}), P, \mu_{\mathrm{co}}\right):=\left(\mathrm{Cl}_{\mathrm{co}}\left(J_{\infty}(\mathfrak{l})\right) \otimes P, d_{\mathrm{co}}\right),
$$

consisting of the followings.

- $\mathrm{Cl}_{\mathrm{co}}\left(J_{\infty}(\mathfrak{l})\right) \otimes P$ denotes the tensor product as graded vertex Poisson algebra (forgetting the differential).

- The differential is given by $d_{\text {co }}:=\left(Q_{\text {co }}\right)_{(0)}+d_{\mathrm{Cl}_{\mathrm{co}}\left(J_{\infty}(\mathfrak{r})\right) \otimes P}$, where the second term is the differential of the tensor product as complex.

(3) Given a coisson momentum map $\mu_{\mathrm{co}}: J_{\infty}(\mathfrak{l}) \rightarrow P$, the cohomology of the coisson BRST complex $\operatorname{BRST}_{\mathrm{co}}\left(J_{\infty}(\mathfrak{l}), P, \mu_{\mathrm{co}}\right)$ is denoted by

$$
H_{\mathrm{co}}^{\frac{\infty}{2}+\bullet}\left(J_{\infty}(\mathfrak{l}), P, \mu_{\mathrm{co}}\right):=H^{\bullet} \operatorname{BRST}_{\mathrm{co}}\left(J_{\infty}(\mathfrak{l}), P, \mu_{\mathrm{co}}\right),
$$

which is a graded Poisson algebra.

Remark 5.1.3. A coisson momentum map $\mu_{\mathrm{co}}$ in our sense is called a chiral momentum map in [A].

The coisson BRST complex satisfies similar properties as in Lemma 2.2.4.

Let $\mathfrak{l}$ be a dg Lie algebra, $R$ be a dg Poisson algebra, and $\mu: \mathfrak{l} \rightarrow R$ be a momentum map. Then we have the symmetric vertex Poisson algebra $J_{\infty}(\operatorname{Sym}(\mathfrak{l}))=\operatorname{Sym}\left(J_{\infty}(\mathfrak{l})\right)$ and the level 0 vertex Poisson algebra $J_{\infty}(R)$, and $\mu$ induces a coisson momentum map

$$
J_{\infty}(\mu): J_{\infty}(\operatorname{Sym}(\mathfrak{l})) \longrightarrow J_{\infty}(R) .
$$

Then we have:

Lemma. For $\mathfrak{l}, R, \mu$ as above, the associated dg Poisson algebra (Definition 4.3.3) of the coisson BRST complex $\operatorname{BRST}_{\text {со }}\left(\mathfrak{l}, J_{\infty}(R), J_{\infty}(\mu)\right)$ is isomorphic to the classical BRST complex $\operatorname{BRST}_{\mathrm{cl}}(\mathfrak{l}, R, \mu)$.

5.2. Coisson gluing procedure. In this part we give an analogue of the discussion in $\S 2.4$ for arc spaces. We basically follow the argument in $[\mathrm{A}, \S 3]$, but with a slight modification. We work over $\mathbb{C}$ here.

As in $\S 2.4$, let $G$ be a simply connected semisimple algebraic group. We denote by $\mathfrak{g}:=\operatorname{Lie}(G)$ the Lie algebra of $G$. We have the affine Poisson scheme $\mathfrak{g}^{*}$ whose coordinate ring is $\operatorname{Sym}(\mathfrak{g})$ with the Kirillov-Kostant Poisson structure.

Let us consider the arc space

$$
J_{\infty}\left(\mathfrak{g}^{*}\right)=\operatorname{Spec}\left(J_{\infty}(\operatorname{Sym}(\mathfrak{g}))\right) .
$$

Recall that the arc space $J_{\infty}(G)$ of $G$ is isomorphic to the proalgebraic group $G[[t]]$ (Lemma 3.1.8). We denote its Lie algebra by $J_{\infty}(\mathfrak{g}):=\mathfrak{g}[[t]]$. The coadjoint action of $G$ on $\mathfrak{g}$ extends to an action of $J_{\infty}(G) \simeq G[[t]]$ on $J_{\infty}\left(\mathfrak{g}^{*}\right)$. Thus, using Notation 2.4.1, we have the category QCoh ${ }^{J_{\infty}(G)}\left(J_{\infty}\left(\mathfrak{g}^{*}\right)\right)$.

On the other hand, by Fact 4.3.8, we can regard $J_{\infty}(\operatorname{Sym}(\mathfrak{g}))$ as a vertex Poisson algebra. Recall Proposition 4.4.10: The category $J_{\infty}(\operatorname{Sym}(\mathfrak{g}))$-VPMod of vertex Poisson modules is equivalent to the category of smooth representations of the Lie algebra $J_{\infty}(\mathfrak{g})=\mathfrak{g}[[t]]$. Then, by the same argument as in $\S 2.4$, we have the equivalence of categories

$$
\mathrm{QCoh}^{J_{\infty}(G)}\left(J_{\infty}\left(\mathfrak{g}^{*}\right)\right) \simeq J_{\infty}(\operatorname{Sym}(\mathfrak{g}))-\mathrm{VPMod}^{\mathrm{lf}}
$$

where $P$-VPMod ${ }^{\text {lf }}$ denotes the full subcategory of $P$-VPMod spanned by those objects on which the adjoint action of $J_{\infty}(\mathfrak{g})$ is locally finite.

Now we have an arc space analogue of Lemma 2.4.3:

Lemma. Let $G$ and $\mathfrak{g}=\operatorname{Lie}(G)$ be as above. A Poisson algebra object in the symmetric monoidal category QCoh ${ }^{J_{\infty}(G)}\left(J_{\infty}\left(\mathfrak{g}^{*}\right)\right)$ is equivalent to a vertex Poisson algebra $P$ equipped with a morphism $\mu_{\text {co }}: \operatorname{Sym}(\mathfrak{g}) \rightarrow P$ of vertex Poisson algebras under which the adjoint action of $J_{\infty}(\mathfrak{g})=\mathfrak{g}[[t]]$ is locally finite.

In view of this lemma, we set:

Definition 5.2.1. A Poisson algebra object in $\mathrm{L}_{Q(0 h}^{J_{\infty}(G)}\left(J_{\infty}\left(\mathfrak{g}^{*}\right)\right)$ is a dg Poisson vertex algebra $P$ equipped with a coisson momentum map $\mu_{P}: J_{\infty}(\operatorname{Sym}(\mathfrak{g})) \rightarrow P$ under which the adjoint action of $J_{\infty}(\mathfrak{g})=\mathfrak{g}[[t]]$ is locally finite. We denote such an object by $\left(P, \mu_{P}\right)$.

Remark. A genuine definition should be given in terms of "homotopy vertex Poisson algebra", which would be the combination of "homotopy vertex Lie algebra" structure and cdga structure. We will come back to this point in a future work. 
We are now interested in a vertex Poisson analogue of the category MT (Definition 2.3.1). It is enough to consider the composition of morphisms. Following Proposition 2.4.4 of the relation between the derived Hamiltonian reduction and the classical BRST complex, we will define the coisson gluing of vertex Poisson algebras by the coisson BRST complex. For that, some preparations are in order:

- Let $\mathfrak{g}_{1}$ and $\mathfrak{g}_{2}$ be the Lie algebras of semisimple algebraic groups $G_{1}$ and $G_{2}$ respectively. For a Poisson algebra object $\left(P, \mu_{P}\right)$ in $\mathrm{L}_{\mathrm{QCoh}}^{J_{\infty}\left(G_{1} \times G_{2}\right)}\left(J_{\infty}\left(\mathfrak{g}_{1}^{*} \times \mathfrak{g}_{2}^{*}\right)\right)$ we define

$$
\mu_{P}^{i}: J_{\infty}\left(\operatorname{Sym}\left(\mathfrak{g}_{i}\right)\right) \longrightarrow P \quad(i=1,2)
$$

by $\mu_{P}^{1}(x)=\mu_{P}(x \otimes 1)$ for $x \in \mathfrak{g}_{1}$ and $\mu_{P}^{2}(y)=\mu_{P}(1 \otimes y)$ for $y \in \mathfrak{g}_{2}$. Here we used $J_{\infty}\left(\operatorname{Sym}\left(\mathfrak{g}_{1} \oplus \mathfrak{g}_{2}\right)\right) \simeq$ $J_{\infty}\left(\operatorname{Sym}\left(\mathfrak{g}_{1}\right)\right) \otimes J_{\infty}\left(\operatorname{Sym}\left(\mathfrak{g}_{2}\right)\right)$.

- For a dg vertex Poisson algebra $P=\left(P, d,|0\rangle, T, Y_{+}, Y_{-}\right)$, we denote by $P^{\text {op }}$ the opposite dg vertex Poisson algebra of $P$. Explicitly, it is given by

$$
P^{\text {op }}:=\left(P, d,|0\rangle,-T, Y_{+}^{\mathrm{op}}, Y_{-}^{\mathrm{op}}\right)
$$

with $Y_{ \pm}^{\mathrm{op}}(a, z):=Y_{ \pm}(a,-z)$.

Definition 5.2.2. Let $\mathfrak{g}_{1}, \mathfrak{g}_{2}$ and $\mathfrak{g}_{3}$ be the Lie algebras of semisimple algebraic groups $G_{1}, G_{2}$ and $G_{3}$ respectively. Let $\left(P, \mu_{P}\right)$ and $\left(P^{\prime}, \mu_{P^{\prime}}\right)$ be Poisson algebra objects in $\mathrm{L}_{Q \operatorname{Coh}}^{G}\left(\mathfrak{g}_{1}^{*} \times \mathfrak{g}_{2}^{*}\right)$ and in $\mathrm{L}_{Q}^{G}$ Coh $\left(\mathfrak{g}_{2}^{*} \times \mathfrak{g}_{3}^{*}\right)$ respectively. We define the Poisson algebra object $P^{\prime} \widetilde{\circ} P$ in $\mathrm{L}_{\mathrm{Q} C o h}^{G}\left(\mathfrak{g}_{1}^{*} \times \mathfrak{g}_{3}^{*}\right)$ by

$$
P^{\prime} \widetilde{\circ} P:=\operatorname{BRST}_{\mathrm{co}}\left(J_{\infty}\left(\mathfrak{g}_{2}\right), P^{\mathrm{op}} \otimes P^{\prime}, \mu_{\mathrm{co}}\right) .
$$

with $\mu_{\mathrm{co}}:=-\mu_{P}^{2}+\mu_{P^{\prime}}^{1}$. The coisson momentum map $J_{\infty}\left(\operatorname{Sym}\left(\mathfrak{g}_{1} \oplus \mathfrak{g}_{3}\right)\right) \rightarrow P^{\prime} \widetilde{\circ} P$ is naturally induced by the given $\mu_{P}^{1}$ and $\mu_{P^{\prime}}^{3}$. We call $P^{\prime} \widetilde{\circ} P$ the coisson gluing of $P$ and $P^{\prime}$.

Here is our definition of vertex Poisson analogue of MT:

Definition 5.2.3. We define the category $\mathrm{MT}_{\text {co }}$ by the following description.

- An object is a simply connected semi-simple algebraic group $G$ over $\mathbb{C}$. We identify it with the associated Lie algebra $\mathfrak{g}$.

- A morphisms from $\mathfrak{g}_{1}$ to $\mathfrak{g}_{2}$ is a Poisson algebra object $\left(P, \mu_{P}\right)$ in $\mathrm{L}_{Q \text { Coh }}^{G}\left(\mathfrak{g}_{1}^{*} \times \mathfrak{g}_{2}^{*}\right)$.

- The composition of $\left(P, \mu_{P}\right) \in \operatorname{Hom}_{\mathrm{MT}_{\mathrm{co}}}\left(\mathfrak{g}_{1}, \mathfrak{g}_{2}\right)$ and $\left(P^{\prime}, \mu_{P^{\prime}}\right) \in \operatorname{Hom}_{\mathrm{MT}_{\mathrm{co}}}\left(\mathfrak{g}_{2}, \mathfrak{g}_{3}\right)$ is given by the coisson gluing $P^{\prime} \widetilde{o} P$.

Let us study the compatibility with the derived gluing (Definition 2.3.1). Recall the monoidal functor $R_{(-)}^{\text {co }}:$ dgVP $\rightarrow$ dgPA taking the associated dg Poisson algebra (Definition 4.3.3). Applying it to a coisson momentum map $\mu_{\mathrm{co}}: J_{\infty}(\mathfrak{g}) \rightarrow P$ and using Lemma 4.3 .9 , we have a morphism $\mu:=R_{\mu_{\mathrm{co}}}^{\text {co }}: \operatorname{Sym}(\mathfrak{g}) \rightarrow R_{P}^{\mathrm{co}}$ of dg Poisson algebra, which is equivalent to a momentum map $\mu: \mathfrak{g} \rightarrow R_{P}^{\text {co }}$. We also have $R_{P \text { op }}^{\text {co }} \simeq\left(R_{P}^{\text {co }}\right)^{\text {op }}$, where the second term denotes the opposite dg Poisson algebra (Definition 2.1.5), and $R_{\mathrm{Cl}}^{\mathrm{co}}\left(J_{\infty}(\mathfrak{g})\right)=\overline{\mathrm{Cl}}(\mathfrak{g})$ by Corollary 4.3.12. Combining these facts, we obtain:

Proposition 5.2.4. Let $P, P^{\prime}$ and $\mu_{\mathrm{co}}: J_{\infty}\left(\operatorname{Sym}\left(\mathfrak{g}_{2}\right)\right) \rightarrow P^{\mathrm{op}} \otimes P^{\prime}$ be as in Definition 5.2.2, and define $\mu=R_{\mu_{\mathrm{co}}}^{\mathrm{co}}: \mathfrak{g}_{2} \rightarrow\left(R_{P}^{\mathrm{co}}\right)^{\mathrm{op}} \otimes R_{P^{\prime}}^{\mathrm{co}}$ as above. Then we have

$$
R_{P^{\prime} \widetilde{\mathrm{o} P}}^{\mathrm{co}} \simeq \operatorname{BRST}_{\mathrm{cl}}\left(\mathfrak{g}_{2},\left(R_{P}^{\mathrm{co}}\right)^{\mathrm{op}} \otimes R_{P^{\prime}}^{\mathrm{co}}, \mu\right)
$$

as dg Poisson algebras.

In particular, combining it with Proposition 2.4.4, we have a quasi-isomorphism of homotopy Poisson algebras

In other words, we have:

$$
R_{P^{\prime} \widetilde{o} P}^{\mathrm{co}} \underset{\text { qis }}{\simeq} R_{P^{\prime}}^{\mathrm{co}} \widetilde{o} R_{P}^{\mathrm{co}}=\left(\left(R_{P}^{\mathrm{co}}\right)^{\mathrm{op}} \otimes R_{P^{\prime}}^{\mathrm{co}}\right) / /_{\mu}^{\mathbb{L}} \operatorname{Sym}(\mathfrak{g}) .
$$

Theorem 5.2.5. The functor $R_{(-)}^{c o}:$ dgVP $\rightarrow$ dgPA taking the associated dg Poisson algebra induces a functor

$$
R_{(-)}^{\mathrm{co}}: \mathrm{MT}_{\text {co }} \longrightarrow \mathrm{MT}
$$

In the remaining part we consider coisson analogue of Hamiltonian reduction of Poisson algebra objects. Recall Definition 2.1.12 of the derived Hamiltonian reduction: For a dg Lie algebra $\mathfrak{l}$, a dg Poisson algebra $R$ and a momentum map $\mu: \mathfrak{l} \rightarrow R$ we have $R / / \mathbb{L}_{\mu}^{\mathbb{L}} \operatorname{Sym}(\mathfrak{l}):=\mathrm{CE}(\mathfrak{l}, \mathbb{k}) \otimes_{\mathrm{CE}(\mathfrak{l}, \operatorname{Sym}(\mathfrak{l}))}^{\mathbb{L}} \operatorname{CE}(\mathfrak{l}, R)$. It is natural to guess that in the coisson setting we replace the $\mathrm{dg}$ Lie algebra $\mathfrak{l}$ by the level 0 vertex Lie algebra $J_{\infty}(\mathfrak{l})$. 
We should be careful here that as a Lie algebra $J_{\infty}(\mathfrak{g})=\mathfrak{g}[[t]]$ is infinite-dimensional. Recalling Proposition 2.2.10, we modify Definition 1.2.13 of Chevalley-Eilenberg complex as

$$
\mathrm{CE}(\mathfrak{g}[[t]], M):=\underline{\operatorname{Hom}}_{U(\mathfrak{g}[[t]])}^{\mathrm{rst}}\left(U\left(\mathfrak{g}[[t]]_{\dagger}\right), M\right)
$$

for a $\operatorname{dg} \mathfrak{g}[[t]]$-module $M$, where $\underline{\operatorname{Hom}}^{\text {rst }}$ is given by Definition 2.2 .9 with the abelian group $\Gamma=\mathbb{Z}$ and the decomposition $\mathfrak{g}[[t]]=\bigoplus_{n \in \mathbb{N}} \mathfrak{g} \otimes t^{n}$. Using this modified Chevalley-Eilenberg complex, we consider:

Definition. For a Poisson algebra object $\left(P, \mu_{\text {co }}\right)$ of $\mathrm{L}_{\mathrm{QCoh}}^{J_{\infty}(G)}\left(J_{\infty}\left(\mathfrak{g}^{*}\right)\right)$, we define a commutative dg algebra

$$
P / / \mu_{\mu_{\mathrm{co}}}^{\mathbb{L}} J_{\infty}(\operatorname{Sym}(\mathfrak{g})):=\operatorname{CE}(\mathfrak{g}[[t]], \mathbb{k}) \bigotimes_{\mathrm{CE}\left(\mathfrak{g}[[t]], J_{\infty}(\operatorname{Sym}(\mathfrak{g}))\right)}^{\mathbb{L}} \mathrm{CE}(\mathfrak{g}[[t]], P),
$$

where $P$ is regarded as a $\mathfrak{g}[[t]]$-module by $\mu_{\mathrm{co}}$. We call it the coisson Hamiltonian reduction of $P$ with respect to the coisson momentum map $\mu_{\mathrm{co}}$.

Then we can apply the argument in Proposition 2.2.10 to the finite-dimensional decomposition $J_{\infty}(\mathfrak{g})=$ $\mathfrak{g}[[t]]=\bigoplus_{n \in \mathbb{N}} \mathfrak{g} \otimes t^{n}$. Since we have $\operatorname{BRST}_{\mathrm{cl}}\left(\mathfrak{g}[[t]], P, \mu_{\mathrm{co}}\right) \simeq \operatorname{BRST}_{\mathrm{co}}\left(J_{\infty}(\mathfrak{g}), P, \mu_{\mathrm{co}}\right)$ as cdgas, we have:

Proposition 5.2.6. Let $G$ and $\mathfrak{g}$ be as above. For a Poisson algebra object $\left(P, \mu_{\mathrm{co}}\right)$ in $\mathrm{L}_{Q \operatorname{coh}}^{J_{\infty}(G)}\left(J_{\infty}\left(\mathfrak{g}^{*}\right)\right)$, we have a quasi-isomorphism of cdgas

$$
P / / \mu_{\mu_{\mathrm{co}}}^{\mathbb{L}} J_{\infty}(\operatorname{Sym}(\mathfrak{g})) \underset{\text { qis }}{\simeq} \operatorname{BRST}_{\mathrm{co}}\left(J_{\infty}(\mathfrak{g}), P, \mu_{\mathrm{co}}\right)
$$

Remark 5.2.7. Let us continue Remark 2.4.5, where we considered the reduction of a Poisson algebra with Hamiltonian $G$-action. Here we consider the action of $J_{\infty}(G)=G[[t]]$ on the arc space instead.

We use the same notation in Remark 2.4.5. Thus, $\left(R, \mu_{R}\right)$ is a Poisson object in $\mathrm{QCoh}^{G}\left(\mathfrak{g}^{*}\right)$, and identified with $\left(X:=\operatorname{Spec}(R), \mu_{X}: X \rightarrow \mathfrak{g}^{*}\right)$. We assume that there is a closed subscheme $S \subset X$ such that the action map gives an isomorphism $G \times S \stackrel{\sim}{\rightarrow} X$, and that the momentum map $\mu_{X}$ is flat. Note that the spectrum $J_{\infty}(R)$ of the arc space $J_{\infty}(X)$ is a Poisson algebra object in QCoh ${ }_{\infty}^{J_{\infty}(G)}\left(J_{\infty}\left(\mathfrak{g}^{*}\right)\right)$, and the corresponding coisson momentum map (Definition 5.1.2) is given by $J_{\infty}\left(\mu_{X}\right): J_{\infty}(X) \rightarrow J_{\infty}\left(\mathfrak{g}^{*}\right)$.

Let $\left(P^{\prime}, \mu_{P^{\prime}}\right)$ be another Poisson algebra object in $\mathrm{QCoh}^{J_{\infty}(G)}\left(J_{\infty}\left(\mathfrak{g}^{*}\right)\right)$, which will be identified with $\left(Y^{\prime}:=\operatorname{Spec}\left(P^{\prime}\right), \mu_{Y^{\prime}}\right)$. Then we can consider the tensor product $J_{\infty}(R)^{\mathrm{op}} \otimes P^{\prime}$ with coisson momentum map $\mu_{\mathrm{co}}: J_{\infty}(X)^{\mathrm{op}} \times Y^{\prime} \rightarrow J_{\infty}\left(\mathfrak{g}^{*}\right), \mu_{\mathrm{co}}(x, y):=-J_{\infty}\left(\mu_{X}\right)(x)+\mu_{Y^{\prime}}(y)$. By the assumption we have $X \simeq G \times S$, so that we also have $J_{\infty}(X) \simeq J_{\infty}(G) \times J_{\infty}(S)$ as schemes. Thus the fiber $\mu_{\mathrm{co}}^{-1}(0)$ is given by

$$
\mu_{\mathrm{co}}^{-1}(0) \simeq J_{\infty}(X) \times_{J_{\infty}\left(\mathfrak{g}^{*}\right)} Y^{\prime} \simeq J_{\infty}(G) \times\left(J_{\infty}(S) \times_{J_{\infty}\left(\mathfrak{g}^{*}\right)} Y^{\prime}\right) .
$$

Now let us further assume that the morphism $J_{\infty}\left(\mu_{X}\right): J_{\infty}(X) \rightarrow J_{\infty}\left(\mathfrak{g}^{*}\right)$ is flat. We have the nonderived Hamiltonian reduction $\left(J_{\infty}(X)^{\mathrm{op}} \times Y^{\prime}\right) / / \Delta\left(J_{\infty}(G)\right)$. Then, similarly as in the argument in Remark 2.4.5, we can deduce from Proposition 5.2.6 that there is an quasi-isomorphism

$$
\operatorname{BRST}_{\mathrm{co}}\left(J_{\infty}(\mathfrak{g}), J_{\infty}(R)^{\mathrm{op}} \otimes P^{\prime}, \mu\right) \simeq\left(R^{\mathrm{op}} \otimes P^{\prime}\right) / / \mu_{\text {co }} J_{\infty}(\operatorname{Sym}(\mathfrak{g})) \simeq \mathbb{k}\left[\mu_{\mathrm{co}}^{-1}(0) / \Delta\left(J_{\infty}(G)\right)\right]
$$

of cdgas, and on the cohomology we have

$$
H^{\bullet} \operatorname{BRST}_{\text {со }}\left(J_{\infty}(\mathfrak{g}), J_{\infty}(R)^{\mathrm{op}} \otimes P^{\prime}, \mu\right) \simeq \mathbb{k}\left[J_{\infty}(S) \times_{J_{\infty}\left(\mathfrak{g}^{*}\right)} X^{\prime}\right] \otimes H^{\bullet}(G, \mathbb{C}) .
$$

Thus we recover the formula $[\mathrm{A},(15)]$.

\section{Derived Gluing of DG Vertex Algebras}

Finally we present the main result. We introduce a vertex algebra analogue $\mathrm{MT}_{\text {ch }}$ of the category MT. The composition of morphisms in $\mathrm{MT}_{\mathrm{ch}}$ will be called the chiral gluing of $\mathrm{dg}$ vertex algebras.

6.1. Chiral BRST complex. In this subsection we explain BRST reduction for vertex algebras. Our exposition is a coordinate-dependent version of the general argument for chiral algebras in [BD, §3.8].

Remark. We give a brief account on the theory of chiral algebras developed by Beilinson and Drinfeld [BD, Chap. 3]. We explained in Remark 5.1.1 that a vertex Poisson algebra is a special case of coisson algebra, which is a Poisson algebra object in the compound $\left(\otimes^{!}\right.$and $\left.\otimes^{\star}\right)$ monoidal structure on the category $\operatorname{DMod}(\operatorname{Ran}(X))$ of $\mathrm{D}$-modules on the Ran space of $X=\mathbb{A}^{1}$. On similar footing, a vertex algebra can be regarded as a Lie algebra object in the chiral monoidal structure $\otimes^{\text {ch }}$ on $\operatorname{DMod}(\operatorname{Ran}(X))$. See also [FG12] for the chiral monoidal structure.

Thus the BRST complex for vertex algebra should be defined by replacing "Lie algebra" in the classical

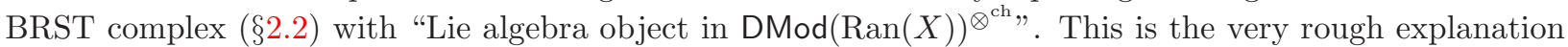
of the construction of chiral BRST complex in [BD, §3.8]. 
6.1.1. Clifford vertex algebra and Tate extension. We work over a field $\mathbb{k}$ of characteristic 0 . In this part we give a new explanation the free fermion vertex algebra $\bigwedge^{\frac{\infty}{2}}(U)$ in $\S 4.1 .6$, and also introduce the Tate extension of dg vertex Lie algebra. These materials are given in [BD, §3.8].

Let $L$ be a dg vertex Lie algebra. We denote by $L^{*}:=\underline{\operatorname{Hom}}(L, \mathbb{k})$ the dual complex. It has a dg vertex Lie algebra structure. The canonical pairing $L^{*} \otimes L \rightarrow \mathbb{k}$ induces a pairing $(\cdot, \cdot)$ on $M:=L^{*}[-1] \oplus L[1]$, and it defines a one-dimensional central extension $M^{b}$ of the commutative $\operatorname{dg}$ vertex Lie algebra $M$. Thus we have the twisted enveloping vertex algebra $U(M)^{b}$ of $M$ (Definition 4.2.6).

Definition ([BD, 3.8.6]). We denote the twisted enveloping vertex algebra by

$$
\mathrm{Cl}_{\mathrm{ch}}(L)=\mathrm{Cl}_{\mathrm{ch}}\left(L, L^{*},(\cdot, \cdot)\right):=U(M)^{b}
$$

and call it the Clifford vertex algebra.

$M^{b}$ has an extra $\mathbb{Z}$-grading by $\left(M^{b}\right)^{(-1)}:=L^{*}[-1],\left(M^{b}\right)^{(0)}:=\mathbb{k}$ and $\left(M^{b}\right)^{(1)}:=L[1]$. It induces an extra $\mathbb{Z}$-grading $\mathrm{Cl}_{\mathrm{ch}}(L)^{(\bullet)}$.

Remark 6.1.1. (1) In $[\mathrm{BD}, 3.8 .6], \mathrm{Cl}_{\mathrm{ch}}(L)$ is called the chiral Clifford algebra, which is the origin of the symbol ch.

(2) The notation $\mathrm{Cl}_{\mathrm{ch}}\left(U((t)), U^{*}((t)) d t,(\cdot, \cdot)\right)$ in Definition 4.1 .25 agrees with this definition. In this case the $\mathbb{Z}$-grading $(\bullet)$ is nothing but the charge grading.

Recall the PBW filtration $\mathrm{Cl}_{\mathrm{ch}}(L)$ • in Definition 4.2.6 (3). We can check that $\mathrm{Cl}_{\mathrm{ch}}(L)_{2}^{(0)}=\left(L^{*}[-1]\right) \otimes$ $(L[1]) \oplus \mathbb{k}$ is a dg vertex Lie subalgebra of $\mathrm{Cl}_{\mathrm{ch}}(L)_{\text {Lie }}$, and is a one-dimensional central extension of $L^{*} \otimes L$. On the other hand, the adjoint action of $\operatorname{Lie}(L)$ on itself yields a morphism $L \rightarrow L^{*} \otimes L$ of dg vertex Lie algebras. We denote by $L^{b}$ the pullback of the extension $0 \rightarrow \mathbb{k}_{\text {Lie }} \rightarrow \mathrm{Cl}_{\mathrm{ch}}(L)_{\text {Lie }} \rightarrow L^{*} \otimes L \rightarrow 0$ by this morphism.

Definition. [BD, 3.8.7] We call the dg vertex Lie algebra $L^{b}$ the Tate extension.

We have a morphism $L^{b} \rightarrow \mathrm{Cl}_{\mathrm{ch}}(L)$ Lie of dg vertex Lie algebras satisfying $1^{b} \mapsto 1_{\mathrm{Cl}_{\mathrm{ch}}(L)}$.

6.1.2. Chiral BRST complex. We continue to work over a field $\mathbb{k}$ of characteristic 0 . We fix a dg vertex Lie algebra $L$.

Definition ([BD, 3.8.8]). A BRST datum is a pair $(V, \alpha)$ of a dg vertex algebra $V$ and a morphism $\alpha: L \rightarrow$ $V_{\text {Lie }}$ of dg Lie algebras such that $\alpha\left(1^{b}\right)=-|0\rangle$, where $|0\rangle$ denotes the vacuum of $V$.

Given a BRST datum $(V, \alpha)$, we have a morphism $\ell^{(0)}=\alpha+\beta: L \rightarrow V \otimes \mathrm{Cl}_{\mathrm{ch}}(L)^{(0)}$ of dg vertex Lie algebras. As in the argument of $\S 5.1$, the contractible complex $L_{\dagger}=(L[1] \rightarrow L)$ inherits the dg vertex Lie algebra structure of $L$. Defining $\ell^{(1)}: L[1] \rightarrow V \otimes \mathrm{Cl}_{\mathrm{ch}}(L)^{(-1)}$ to be the composition $L[1] \hookrightarrow \mathrm{Cl}_{\mathrm{ch}}(L)^{(-1)} \hookrightarrow$ $V \otimes \mathrm{Cl}_{\mathrm{ch}}(L)^{(-1)}$, we have a morphism

$$
l: L_{\dagger} \longrightarrow \mathrm{Cl}_{\mathrm{ch}}(L) \otimes V
$$

of graded vertex Lie algebras (forgetting the differential).

We now regard the symmetric dg algebra $\operatorname{Sym}\left(L^{*}[-1]\right)$ as a Chevalley-Eilenberg complex of the trivial $L$-module, and denote by $\delta$ the differential. By the embedding $\operatorname{Sym}\left(L^{*}[-1]\right) \subset \mathrm{Cl}_{\mathrm{ch}}(L) \subset V \otimes \mathrm{Cl}_{\mathrm{ch}}(L)$ we regard $\delta$ acting on $V \otimes \mathrm{Cl}_{\mathrm{ch}}(L)$.

Fact 6.1.2 ([BD, 3.8.10]). There is a unique element $Q \in V \otimes \mathrm{Cl}_{\mathrm{ch}}(L)^{(1)}$ of cohomological degree 1 such that $\left[Q_{(0)}, l^{(-1)}\right]=l^{(0)}$ and $Q_{(0)}^{2}=0$. The morphism $d_{\mathrm{ch}}:=Q_{(0)}+d_{\mathrm{Cl}_{\mathrm{ch}}(L) \otimes V}$ defines a dg vertex algebra structure on $V \otimes \mathrm{Cl}_{\mathrm{ch}}(L)$. We denote it by

$$
\operatorname{BRST}_{\mathrm{ch}}(V, \alpha):=\left(V \otimes \mathrm{Cl}_{\mathrm{ch}}(L), d_{\mathrm{ch}}\right)
$$

and call it the (chiral) BRST complex for the BRST datum $(V, L, \alpha)$.

The morphism $l$ gives a morphism $L_{\dagger} \rightarrow \operatorname{BRST}_{\mathrm{ch}}(V, \alpha)_{\text {Lie }}$ of dg Lie algebras.

6.1.3. The case of universal affine vertex algebras. In this part we describe in detail the chiral BRST complex for the universal affine vertex algebras, which will be used to formulate the vertex algebra analogue of the category MT in $\S 6.2$. Hereafter we work over $\mathbb{C}$, and use notations in $\S 4.1 .4$ for Lie algebras. In particular

- $\mathfrak{g}$ is the Lie algebra of the semi-simple algebraic group over $\mathbb{C}$, and

- $\widehat{\mathfrak{g}}=\mathfrak{g}((t)) \oplus \mathbb{C} K$ is the the derived algebra of the non-twisted affine Lie algebra associated to $\mathfrak{g}$. 
Let $V_{k}(\mathfrak{g})$ be the universal affine vertex algebra at level $k \in \mathbb{C}$. (Definition 4.1.13). Regarding it as a graded dg vertex algebra concentrated in cohomological degree 0 with trivial differential (Definition 4.1.23), we have the category $V_{k}(\mathfrak{g})$-dgVMod of dg $V_{k}(\mathfrak{g})$-modules (Definition 4.1.21). Hereafter we regard $\mathfrak{g} \subset V_{k}(\mathfrak{g})$ by the injective linear map

$$
\mathfrak{g} \longleftrightarrow V_{k}(\mathfrak{g}), \quad x \longmapsto x t^{-1}|0\rangle .
$$

Following $\left[\mathrm{A}, \S 3\right.$, p. 10], we introduce the notion of momentum maps in the category $V_{k}(\mathfrak{g})$-dgVMod:

Definition 6.1.3. $\quad$ (1) A chiral momentum map is a morphism $\mu: V_{k}(\mathfrak{g}) \rightarrow V$ of dg vertex algebras.

(2) A dg vertex algebra object (dgva object for short) in $V_{k}(\mathfrak{g})$-dgVMod is a pair $(V, \mu)$ of a dg vertex algebra $V$ and a chiral momentum map $\mu: V_{k}(\mathfrak{g}) \rightarrow V$, where we regard $V \in V_{k}(\mathfrak{g})$-dgVMod by Lemma 4.1.24.

Remark 6.1.4. In $[A]$ a chiral momentum map is called a chiral quantum momentum map. See also Remark 5.1.3.

Given a chiral momentum map $\mu: V_{k}(\mathfrak{g}) \rightarrow V$, we have a morphism $\mu_{\text {Lie }}: V_{k}(\mathfrak{g}) \rightarrow V_{\text {Lie }}$ of dg vertex Lie algebras by the polar part construction (Lemma 4.2.3). Restricting $\mu_{\text {Lie }}$ to the subalgebra $v_{k}(\mathfrak{g}) \subset V_{k}(\mathfrak{g})$ in Example 4.2.4, we have a BRST datum $\left(V,\left.\mu_{\text {Lie }}\right|_{v_{k}(\mathfrak{g})}: v_{k}(\mathfrak{g}) \rightarrow V\right)$, and thus we have the BRST complex (Fact 6.1.2).

Definition 6.1.5. Let $k \in \mathbb{C}$ and $(V, \mu)$ be a dgva object in $V_{k}(\mathfrak{g})$-dgVMod.

(1) We denote the BRST complex for the $\operatorname{BRST}$ datum $\left(V,\left.\mu_{\text {Lie }}\right|_{v_{k}(\mathfrak{g})}\right)$ by

$$
\operatorname{BRST}\left(\mathfrak{g}_{k}, V, \mu\right):=\operatorname{BRST}_{\mathrm{ch}}\left(V,\left.\mu_{\text {Lie }}\right|_{v_{k}(\mathfrak{g})}\right) \text {. }
$$

(2) The cohomology vertex algebra (Lemma 4.1.22) of the BRST complex is denoted by

$$
H^{\frac{\infty}{2}+\bullet}\left(\widehat{\mathfrak{g}}_{k}, V, \mu\right):=H^{\bullet}\left(\operatorname{BRST}\left(\widehat{\mathfrak{g}}_{k}, V, \mu\right), d_{\mathrm{cl}}\right),
$$

and called the BRST cohomology.

(3) In the case $V=V_{k}(\mathfrak{g})$ and $\mu=$ id, we denote $\operatorname{BRST}\left(\widehat{\mathfrak{g}}_{k}, V_{k}(\mathfrak{g})\right):=\operatorname{BRST}\left(\widehat{\mathfrak{g}}_{k}, V_{k}(\mathfrak{g})\right.$,id).

Now recall the free fermionic dg vertex algebra $\Lambda^{\frac{\infty}{2}}(\mathfrak{g})$ in Definition 4.1.25. Note that the $\mathbb{Z}$-grading $\Lambda^{\frac{\infty}{2}}(\mathfrak{g}) \bullet$ of the dg structure is equal to the minus of the charge grading $\Lambda^{\frac{\infty}{2}+\bullet}(\mathfrak{g})$. By the characterization of the BRST charge in Fact 6.1.2, we can write down this BRST complex in the following form:

Lemma 6.1.6. Let $k \in \mathbb{C}$ and $(V, \mu)$ be a dgva object in $V_{k}(\mathfrak{g})$-dgVMod. Then the $\operatorname{dg}$ vertex algebra $\operatorname{BRST}\left(\widehat{\mathfrak{g}}_{k}, V, \mu\right)$ is described as follows.

(1) As a graded vertex algebra (forgetting the differential), we have

$$
\operatorname{BRST}\left(\widehat{\mathfrak{g}}_{k}, V, \mu\right) \simeq V \otimes \Lambda^{\frac{\infty}{2}}(\mathfrak{g}) .
$$

(2) The differential is given by $d_{\mathrm{ch}}=Q_{(0)}+d_{V \otimes \Lambda^{\infty / 2}(\mathfrak{g})}$, where $Q \in \operatorname{BRST}\left(\widehat{\mathfrak{g}}_{k}, V, \mu\right)$ is the BRST charge

$$
Q:=\sum_{i=1}^{\operatorname{dim} \mathfrak{g}} \mu\left(x_{i}\right) \otimes \psi_{i}^{*}-\frac{1}{2} \sum_{i, j, k=1}^{\operatorname{dim} \mathfrak{g}} 1 \otimes c_{i j}^{k} \psi_{i}^{*} \psi_{j}^{*} \psi_{k} .
$$

Here we used the structure constant $c_{i j}^{k}$ of $\mathfrak{g}$ as in Lemma 2.2.5 and omit the vacuum $|0\rangle$.

Now recall the functor taking the associated graded space of the Li filtration (Lemma 4.4.4):

$$
\operatorname{gr}^{F}: \mathrm{dgVA} \longrightarrow \operatorname{dgVP} \text {. }
$$

Given a dgva object $\left(V, \mu_{V}\right)$ in $V_{k}(\mathfrak{g})$-dgVMod. the chiral momentum map $\mu_{V}: V_{k}(\mathfrak{g}) \rightarrow V$ induces the coisson momentum map

$$
\mu_{\mathrm{gr}^{F} V}: \operatorname{gr}^{F} V_{k}(\mathfrak{g}) \simeq J_{\infty}(\operatorname{Sym}(\mathfrak{g})) \longrightarrow \operatorname{gr}^{F} V .
$$

Here we used the isomorphism in Fact 4.4.9 (2). Then, comparing the description of BRST complex (Lemma 6.1.6) with the coisson BRST complex (Definition 5.1.2), we obtain:

Lemma 6.1.7. Let $\left(V, \mu_{V}\right)$ be a dgva object in $V_{k}(\mathfrak{g})$-dgVMod with $k \in \mathbb{C}$. Then we have an isomorphism of dg vertex Poisson algebras

$$
\operatorname{gr}^{F} \operatorname{BRST}\left(\widehat{\mathfrak{g}}_{k}, V, \mu_{V}\right) \simeq \operatorname{BRST}_{\text {co }}\left(J_{\infty}(\mathfrak{g}), \operatorname{gr}^{F} V, \mu_{\operatorname{gr}^{F} V}\right) .
$$


6.2. Chiral gluing procedure. In this subsection we give a vertex algebra analogue of composition of morphisms in the category MT. We work under the same setting as in $\S 6.1 .3$, and use the same symbols $\mathfrak{g}=\operatorname{Lie}(G), V_{k}(\mathfrak{g})$ and so on.

Some preparations are in order.

- Let $(V, \mu)$ and $\left(V^{\prime}, \mu^{\prime}\right)$ be dgva objects in $V_{k}(\mathfrak{g})$-dgVMod and $V_{l}(\mathfrak{g})$-dgVMod respectively. Then we can regard $\left(V \otimes V^{\prime}, \mu \otimes \mu^{\prime}\right) \in V_{k+l}(\mathfrak{g})$-dgVMod by the diagonal action of $\widehat{\mathfrak{g}}$ (Lemma 4.1.17).

- For a dg vertex algebra $V=\left(V^{\bullet}, d,|0\rangle, T, Y\right)$, we define the opposite dg vertex algebra $V^{\text {op }}$ by

$$
V^{\mathrm{op}}:=\left(V^{\bullet}, d,|0\rangle, T^{\mathrm{op}}, Y^{\mathrm{op}}\right)
$$

with $Y^{\mathrm{op}}(a, z):=Y(a,-z)$ and $T^{\mathrm{op}}:=-T$. We have

$$
\operatorname{gr}^{F}\left(V^{\mathrm{op}}\right) \simeq\left(\operatorname{gr}^{F} V\right)^{\mathrm{op}}
$$

as dg vertex Poisson algebras.

- For a dgva object $(V, \mu)$ in $V_{k}(\mathfrak{g})$-dgVMod, we have the new dgva object $\left(V^{\mathrm{op}}, \mu^{\mathrm{op}}\right)$ with $\mu^{\mathrm{op}}(a):=$ $-\mu(a)$.

Definition 6.2.1. Let $\left(V, \mu_{V}\right)$ and $\left(V^{\prime}, \mu_{V^{\prime}}\right)$ be dgva objects in $V_{k}(\mathfrak{g})$-dgVMod and in $V_{l}(\mathfrak{g})$-dgVMod respectively. We define a chiral momentum map $\mu: V^{\mathrm{op}} \otimes V^{\prime} \rightarrow V_{k+l}(\mathfrak{g})$ by $\mu(a \otimes b):=-\mu_{V}(a)+\mu_{V^{\prime}}(b)$, and a dgva object $V^{\prime} \widetilde{o} V$ in $V_{k+l}(\mathfrak{g})$-dgVMod by

$$
V^{\prime} \widetilde{\circ} V:=\operatorname{BRST}\left(\widehat{\mathfrak{g}}_{k+l}, V^{\mathrm{op}} \otimes V^{\prime}, \mu\right)
$$

and call it the chiral gluing.

Recall that for a dg vertex algebra $V$, we denote by $R_{V}=F^{0} V / F^{1} V$ Zhu's $C_{2}$-algebra (Definition 4.4.5). By Lemma 6.1.7 and Definition 5.2.2 of the coisson gluing, we have:

Proposition 6.2.2. Under the same setting of Definition 6.2.1, we have the following quasi-isomorphism of dg vertex Poisson algebras:

On Zhu's $C_{2}$-algebras, we have

$$
\operatorname{gr}^{F}\left(V^{\prime} \widetilde{\circ} V\right) \simeq\left(\operatorname{gr}^{F} V^{\prime}\right) \widetilde{\circ}\left(\operatorname{gr}^{F} V\right)
$$

$$
R_{V^{\prime} \widetilde{\circ} V} \simeq R_{V^{\prime}} \widetilde{\circ} R_{V} .
$$

6.3. The category $\mathrm{MT}_{\mathrm{ch}}$. Under the same setting as in $\S 6.2$, we finally introduce the category $\mathrm{MT}_{\mathrm{ch}}$, which is a vertex algebra analogue of MT in $\S 2.3$ and $\mathrm{MT}_{\text {co }}$ in $\S 5.2$. In the latter case, we considered a vertex Poisson object in $\mathrm{L}_{\mathrm{QCoh}}^{J_{\infty}(G)}\left(J_{\infty}\left(\mathfrak{g}^{*}\right)\right)$, which satisfies some finite condition (Definition 5.2.1). Following [A, $\S 3$, p. 10], we introduce the corresponding category of vertex algebras.

Recall the equivalence of $V_{k}(\mathfrak{g})$-modules and smooth $\widehat{\mathfrak{g}}$-representations of level $k$ (Fact 4.1.16). Thus on a $V_{k}(\mathfrak{g})$-module we can discuss the action of $\mathfrak{g} \subset \widehat{\mathfrak{g}}$ and $\mathfrak{g}[[t]] t \subset \widehat{\mathfrak{g}}$.

Definition. Let $k \in \mathbb{C}$.

(1) We denote by $\operatorname{dgKL} L_{k}(\mathfrak{g})$ the full subcategory of $V_{k}(\mathfrak{g})$-dgVMod spanned by objects on which $\mathfrak{g}[[t]] t$ acts locally nilpotently and $\mathfrak{g}$ acts locally finitely.

(2) We denote by $\mathrm{KL}_{k}(\mathfrak{g})$ the full subcategory of $\operatorname{dgKL}_{k}(\mathfrak{g})$ spanned by objects concentrated in cohomological degree 0 .

(3) We denote by $\mathrm{KL}_{k}^{\text {ord }}(\mathfrak{g})$ the full subcategory of $\mathrm{KL}_{k}(\mathfrak{g})$ spanned by objects that are $\mathbb{N}$-graded (Definition 4.1.11) and each homogeneous subspaces are finite-dimensional.

Remark. (1) The subcategory $\mathrm{KL}_{k}(\mathfrak{g}) \subset \mathrm{dgKL}_{k}(\mathfrak{g})$ spanned by those dg modules concentrated in degree 0 was originally introduced in [A12] as the category of graded Harish-Chandra $(\widehat{\mathfrak{g}}, G[[t]])$-modules of level $k$. The category $\mathrm{KL}_{k}(\mathfrak{g})$ was used for showing the cohomology vanishing of BRST complex in [A12], and used in [A] to construct the genus zero chiral algebra $\mathbf{V}_{G, b}^{\mathcal{S}}$ of class $\mathcal{S}$.

(2) As noted in $[A, \S 3]$, every object in $\mathrm{KL}_{k}(\mathfrak{g})$ is a colimit of a direct system of objects in $\mathrm{KL}_{k}^{\text {ord }}(\mathfrak{g})$.

We immediately have:

Lemma 6.3.1. Let $V$ be a vertex algebra object in $\operatorname{dgKL}_{k}(\mathfrak{g})$. Then $\operatorname{gr}^{F}$ is a Poisson algebra object in $\mathrm{L}_{Q}^{J_{\infty}(G)}\left(J_{\infty}\left(\mathfrak{g}^{*}\right)\right)$, and $R_{V}$ is a Poisson algebra object in $\mathrm{L}_{Q}^{G} \operatorname{Coh}^{\prime}\left(\mathfrak{g}^{*}\right)$,

The family $\left\{\operatorname{dgKL}_{k}(\mathfrak{g}) \mid k \in \mathbb{C}\right\}$ inherits the tensor structure of $\left\{V_{k}(\mathfrak{g})\right.$-dgVMod $\left.\mid k \in \mathbb{C}\right\}$ in Lemma 4.1.17: For $M \in \operatorname{dgKL}_{k}(\mathfrak{g})$ and $N \in \operatorname{dgKL}_{k}(\mathfrak{g})$, we have $M \otimes N \in \mathrm{dgKL}_{k+l}(\mathfrak{g})$ by the diagonal action of $\widehat{\mathfrak{g}}$.

Here is the definition of vertex analogue of the category MT. 
Definition 6.3.2. We define the category $\mathrm{MT}_{\mathrm{ch}}$ by the following description.

- An object is a simply connected semi-simple algebraic group $G$ over $\mathbb{C}$. We identify it with the associated Lie algebra $\mathfrak{g}$.

- A morphisms from $\mathfrak{g}_{1}$ to $\mathfrak{g}_{2}$ is a vertex algebra object $\left(V, \mu_{V}\right)$ in $\left(V_{k}\left(\mathfrak{g}_{1}\right) \otimes V_{l}\left(\mathfrak{g}_{2}\right)\right)$-dgVMod with some $k, l \in \mathbb{C}$ such that $\left(V, \mu_{V}^{1}\right) \in \operatorname{dgKL}_{k}\left(\mathfrak{g}_{1}\right)$ and $\left(V, \mu_{V}^{2}\right) \in \operatorname{dgKL}_{l}\left(\mathfrak{g}_{2}\right)$. Here we defined $\mu_{V}^{1}: V_{k}\left(\mathfrak{g}_{1}\right) \rightarrow V$, $\mu_{V}^{1}(a):=\mu_{V}(a \otimes|0\rangle)$ and $\mu_{V}^{2}: V_{l}\left(\mathfrak{g}_{2}\right) \rightarrow V, \mu_{V}^{2}(b):=\mu_{V}(|0\rangle \otimes b)$.

- The composition of $\left(V, \mu_{V}\right) \in \operatorname{Hom}_{\mathrm{MT}_{\mathrm{ch}}}\left(\mathfrak{g}_{1}, \mathfrak{g}_{2}\right)$ and $\left(V^{\prime}, \mu_{V^{\prime}}\right) \in \operatorname{Hom}_{\mathrm{MT}_{\mathrm{ch}}}\left(\mathfrak{g}_{2}, \mathfrak{g}_{3}\right)$ is given by the chiral gluing $V^{\prime} \widetilde{\circ} V$ in Definition 6.2.1 where we regard $\left(V, \mu_{V}^{2}\right) \in V_{l}\left(\mathfrak{g}_{2}\right)$-dgVMod and $\left(V, \mu_{V^{\prime}}^{1}\right) \in$ $V_{k^{\prime}}\left(\mathfrak{g}_{2}\right)$-dgVMod.

By Proposition 6.2.2 and Lemma 6.3.1 we have the main result:

Theorem 6.3.3. The functors $\operatorname{gr}^{F}: \operatorname{dgVA} \rightarrow \operatorname{dgVP}, R: \operatorname{dgVA} \rightarrow \operatorname{dgPA}$ and $R: \operatorname{dgVP} \rightarrow \operatorname{dgPA}$ give a commutative diagram

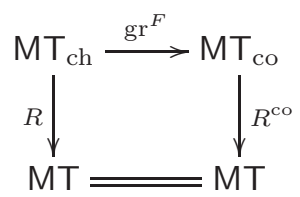

Remark 6.3.4. Let us continue Remark 5.2.7 and give a connection to the argument in [A, §3]. Recall that in $§ 5.2$ we considered coisson gluing, which is a vertex Poisson analogue of composition of morphisms in MT. Here we consider a chiral analogue.

Let $\left(V, \mu_{V}\right)$ be a vertex algebra object in $\mathrm{KL}_{k}$. Then the associated graded space $\mathrm{gr}^{F} V$ is a vertex Poisson algebra object in QCoh ${ }^{J_{\infty}(G)}\left(J_{\infty}\left(\mathfrak{g}^{*}\right)\right)$, and Zhu's $C_{2}$-algebra $R_{V}$ is a Poisson algebra object in QCoh ${ }^{G}(\mathfrak{g})$. Let $X=\operatorname{Spec}(R)$ be an affine Poisson scheme, and assume the following conditions.

(i) $R_{V} \simeq R$.

(ii) The surjection $J_{\infty}\left(R_{V}\right) \rightarrow \operatorname{gr}^{F} V$ of vertex Poisson algebras is an isomorphism.

If moreover $V$ is separated (Definition 4.4.8), then $V$ is called a strict chiral quantization of $X$ [A, Definition 2.1]. We further assume the following conditions considered in Remarks 2.4.5 and 5.2.7.

(iii) There is a closed subscheme $S \subset X$ such that the action map gives an isomorphism $G \times S \stackrel{\sim}{\rightarrow} X$.

(iv) The chiral momentum map $J_{\infty}\left(\mu_{X}\right)=\left(\operatorname{gr}^{F} \mu_{V}\right)^{*}: J_{\infty}(X) \rightarrow J_{\infty}\left(\mathfrak{g}^{*}\right)$ is flat.

Let $\left(V^{\prime}, \mu_{V^{\prime}}\right)$ be a vertex algebra object in $\mathrm{KL}_{l}$. Then the tensor product $V^{\text {op }} \otimes V^{\prime}$ is a vertex algebra object in $\mathrm{KL}_{k+l}$ with chiral momentum map $\mu(a, b):=-\mu_{V}(a)+\mu_{V^{\prime}}(b)$.

By the same argument in Remarks 2.4.5 and 5.2.7, we can then deduce an quasi-isomorphism

$$
\operatorname{gr}^{F} \operatorname{BRST}\left(\widehat{\mathfrak{g}}_{k+l}, V^{\mathrm{op}} \otimes V^{\prime}, \mu\right) \simeq\left(\left(J_{\infty}(R)\right)^{\mathrm{op}} \otimes\left(\operatorname{gr}^{F} V^{\prime}\right)\right) / / \operatorname{gr}^{F} \mu J_{\infty}\left(\mathfrak{g}^{*}\right) \simeq \mathbb{k}\left[\left(\operatorname{gr}^{F} \mu\right)^{-1}(0) / \Delta\left(J_{\infty}(G)\right)\right]
$$

of cdgas, and as for the cohomology we have

$$
\operatorname{gr}^{F} H^{\frac{\infty}{2}+\bullet}\left(\widehat{\mathfrak{g}}_{k+l}, V^{\mathrm{op}} \otimes V^{\prime}, \mu\right) \simeq\left(\mathbb{k}\left[J_{\infty}(S)\right] \otimes_{J_{\infty}(\operatorname{Sym}(\mathfrak{g}))} \operatorname{gr}^{F} V^{\prime}\right) \otimes H^{\bullet}(G, \mathbb{C}) .
$$

Thus we have

$$
\operatorname{gr}^{F} H^{\frac{\infty}{2}+0}\left(\widehat{\mathfrak{g}}_{k+l}, V^{\mathrm{op}} \otimes V^{\prime}, \mu\right) \simeq \mathbb{k}\left[J_{\infty}(S)\right] \otimes_{J_{\infty}(\operatorname{Sym}(\mathfrak{g}))} \operatorname{gr}^{F} V^{\prime},
$$

which recovers the formula in [A, Theorem 3.1].

\section{REFERENCES}

[A07] T. Arakawa, Representation theory of $W$-algebras, Invent. math., 169 (2007), 219-320.

[A12] T. Arakawa, A remark on the $C_{2}$ cofiniteness condition on vertex algebras, Math. Z., 270, (2012), no. 1-2, 559-575.

[A15] T. Arakawa, Associated Varieties of Modules Over Kac-Moody Algebras and C ${ }_{2}$-Cofiniteness of W-Algebras, Int. Math. Res. Not., 2015 (2015). 11605-11666.

[A] T. Arakawa, Chiral algebras of class $\mathcal{S}$ and Moore-Tachikawa symplectic varieties, preprint, arXiv:1811.01577v2.

[AM] T. Arakawa, A. Moreau, Arc spaces and chiral symplectic cores, to appear in the special issue of Publ. Res. Inst. Math. in honor of Professor Masaki Kashiwara's 70th birthday; arXiv:1802.06533v2.

[BD] Beilinson, A., Drinfeld, V., Chiral algebras, American Mathematical Society Colloquium Publications, 51, American Mathematical Society, Providence, RI, 2004.

[BLR] S. Bosch, W. Lütkebohmert, M. Raynaud, Néron models, Ergebnisse Math. Grenzgebiete 3. Folge, 21, SpringerVerlag, 1990.

[BFN] A. Braverman, M. Finkelberg, H. Nakajima, Ring objects in the equivariant derived Satake category arising from coulomb branches, preprint, arXiv:1706.02112v5.

$\left[\mathrm{BLL}^{+}\right]$C. Beem, M. Lemos, P. Liendo, W. Peelaers, L. Rastelli, B.C. van Rees, Infinite chiral symmetry in four dimensions, Comm. Math. Phys., 336 (2015), no. 3, 1359-1433.

[BPRvR] C. Beem, W. Peelaers, L. Rastelli, B.C. van Rees, Chiral algebras of class S, J. High Energy Phys., 5 (2015), 020. 
[BR18] C. Beem, L. Rastelli, Vertex operator algebras, Higgs branches, and modular differential equations, J. High Energy Phys., 8 (2018), 114.

[C14] D. Calaque, Three lectures on derived symplectic geometry and topological field theories, Indag. Math., (N.S.) 25 (2014), no. 5, 926-947.

[C15] D. Calaque, Lagrangian structures on mapping stacks and semi-classical TFTs, in Stacks and categories in geometry, topology, and algebra, 1-23, Contemp. Math., 643, Amer. Math. Soc., 2015; arXiv:1306.3235.

[CPT+] D. Calaque, T. Pantev, B. Toën, M. Vaquié. G. Vezzosi, Shifted Poisson structures and deformation quantization, J. Top., 10 (2017), 483-584.

[EM09] L. Ein, M. Mustaţă, Jet schemes and singularities, in Algebraic geometry-Seattle 2005, Part 2, 80 Proc. Sympos. Pure Math., 505-546. Amer. Math. Soc., Providence, RI, 2009.

[FBZ] E. Frenkel, D. Ben-Zvi, Vertex algebras and algebraic curves, 2nd ed., Math. Surv. Monog., 88, Amer. Math. Soc., 2004.

[FG12] J. Francis, D. Gaitsgory, Chiral Koszul duality, Sel. Math. New Ser., 18 (2012), 27-87.

[Ka] V. Kac, Vertex algebras for beginners, 2nd ed., Univ. Lect. Ser., 10, Amer. Math. Soc., 1998

[KS87] B. Kostant, S. Sternberg, Symplectic reduction, BRS cohomology, and infinite-dimensional Clifford algebras, Ann. Physics, 176 (1) (1987), 49-113.

[Ku15] T. Kuwabara, BRST cohomologies for symplectic reflection algebras and quantizations of hypertoric varieties, Transformation Groups 20, no. 2 (2015) 437-461.

[LPV] C. Laurent-Gengoux, A. Pichereau, P. Vanhaecke, Poisson Structures, Grundlehren math. Wiss., 347, SpringerVerlag, 2013.

[Li05] H. Li, Abelianizing vertex algebras, Commun. Math. Phys., 259 (2005), no. 2, 391-411.

[LV] J-L. Loday, B. Vallette, Algebraic operads, Grundlehren Math. Wiss., 346, Springer, 2012.

[Lu1] J. Lurie, Higher topos theory, Annals of Mathematics Studies 170, Princeton University Press, Princeton, NJ, 2009.

[Lu2] J. Lurie, Higher algebra, September 2017, available at his webpage https://www.math.ias.edu/ lurie/

[M16] V. Melani, Poisson bivectors and Poisson brackets on affine derived stacks, Adv. Math., 288 (2016), 1097-1120.

[MS18] V. Melani, P. Safronov, Derived coisotropic structures II: stacks and quantization, Sel. Math. New Ser., 24 (2018) 3119-3173.

[MT12] G. W. Moore, Y. Tachikawa. On 2d TQFTs whose values are holomorphic symplectic varieties, in String-Math 2011, Proc. Sympos. Pure Math., 85 191-207. Amer. Math. Soc., 2012.

[PTVV] T. Pantev, B. Toën, M. Vaquie, G. Vezzosi, Shifted symplectic structures, Publ. math. IHES 117 (2013), $271-328$.

[S17] P. Safronov, Poisson reduction as a coisotropic intersection, Higher Structures 1 (2017), 87-121.

[S] P. Safronov, Lectures on shifted Poisson geometry, arXiv:1709.07698.

[SP] The Stacks Project Authors Stacks Project, an open source textbook and reference work on algebraic geometry, https://stacks.math. columbia.edu.

[Ta18] Y. Tachikawa, On 'categories' of quantum field theories, Proc. Int. Cong. of Math., Rio de Janeiro, 2:2695-2718, 2018.

[T14] B. Toën, Derived algebraic geometry, EMS Surv. Math. Sci. 1 (2014), 153-240.

[TVe] B. Toën, G. Vezzosi, Homotopical Algebraic Geometry II: Geometric Stacks and Applications, Mem. Amer. Math. Soc., 193, 2008.

[Z96] Y. Zhu, Modular invariance of characters of vertex operator algebras, J. Amer. Math. Soc., 9 (1996), no. 1, 237-302.

Graduate School of Mathematics, Nagoya University. Furocho, Chikusaku, Nagoya, Japan, $464-8602$.

E-mail address: yanagida@math.nagoya-u.ac.jp 